\title{
Personenbezogene Merkmale von Social Entrepreneuren (SE), Einstellungen \& Motive
}

Für die Kategorie „Personenbezogene Merkmale von SE, Einstellungen und Motive" lassen sich folgende Unterkategorien herausarbeiten, die in dieser Reihenfolge dargestellt und diskutiert werden:

- motivationale Aspekte sozialunternehmerischen Handelns,

- Motive und Phasen des Motivationsprozesses,

- Stellenwert von Bedürfnissen und Werten im Motivationsprozess,

- Positionierung gegenüber dem Geldverdienen,

- persönliche finanzielle Absicherung,

- begriffliche Determinanten zur SE(O)-Zugehörigkeit,

- Anspruchsniveau Problemlösung,

- Anspruchsniveau Skalierung,

- Anspruchsniveau Innovationsentwicklung,

- Zielsetzung und Zielerreichung,

- Stellenwert des Unternehmertums im Verhältnis zur sozialen Zielsetzung,

- konzeptionelle (Un)Vereinbarkeit von sozialer Zielsetzung und Unternehmertum sowie

- Hinweise zur Persönlichkeitstypologie von Social Entrepreneuren, wobei diese durch Limitationen geprägt sind.

Elektronisches Zusatzmaterial Die elektronische Version dieses Kapitels enthält Zusatzmaterial, das berechtigten Benutzern zur Verfügung steht https://doi.org/10.1007/978-3-658-35145-8_8. 
Zur Illustration werden dabei Schilderungen der Interviewpartner zu generierten Kodes wieder als Endnotenverweis eingearbeitet, und die gewonnenen Erkenntnisse werden an internationalen Studien, die im Forschungskontext der Arbeitsgestaltung und der Wirkung von Arbeit von Social Entrepreneuren bestehen, gespiegelt und in der bestehenden Forschungslandschaft verortet.

Bei der Diskussion werden abermals wissenschaftliche Erkenntnisse wie z. B. Modelle und Theorien herangezogen, die zur inhaltlichen Erläuterung der eigenen Studienergebnisse passen und die Bedeutung der Kodes insofern untermauern. Darüber hinaus werden in diesem Kapitel die Erkenntnisse in ein motivationspsychologisches und in ein persönlichkeitspsychologisches Grundverständnis eingebunden. Dies erfordert, dass zuvor die entsprechenden psychologischen Modelle und Theorien hinreichend hergeleitet werden.

Kapitelabschließend wird aufgezeigt, welche Bedeutung die in diesem Kapitel dargestellten Erkenntnisse für das Wirkungsmodell zur Arbeitsgestaltung von SE besitzen.

\subsection{Einführung in motivationale Aspekte sozialunternehmerischen Handelns}

Das Wort Motivation leitet sich ab aus dem lateinischen Begriff motivans: bewegend/sich von der Stelle bewegend. Die Motivationspsychologie widmet sich den personenbezogenen Beweggründen, eine Handlung auszulösen bzw. selbst in Handlung zu kommen und Verhalten zu gestalten. Dabei bedarf jede Motivationsform einer situativ passenden Gelegenheit im Sinne einer Handlungsplattform, auf der basierend eigenes Verhalten gezeigt werden kann.

Äußerungen von Befragten lassen sich zuordnen zur motivationspsychologisch grundsätzlich gegebenen Unterscheidung zwischen intrinsischer Motivation, die in der Freude an einer thematischen Auseinandersetzung begründet ist (IPÄ ${ }^{639}$; IPS $^{640}$, i.V. m. IPS ${ }^{641}$; IP10 ${ }^{642}$; FG5Z1637-1673 ${ }^{643}$; IP35 ${ }^{644}$; IP18 ${ }^{645}$ ), und extrinsischer Motivation, die als Mittel zum Zweck funktioniert und sich dementsprechend instrumentell begründen lässt (IP10 ${ }^{646}$; IP $\pi^{647}$; in nicht gegebener Ausprägung betont z. B. bei $\left.\mathrm{IP}^{648}\right)$. Die Differenzierung geht zurück auf Herzberg (1959; siehe ebenso z. B. Deci, Connell \& Ryan, 1989; Deci, Koestner \& Ryan, 1999; Deci \& Ryan, 2000; Gagné, Forest, Gilbert, Aube, Morin \& Malorni, 2010). Erfolgserlebnisse werden bei Befragten zum Nährboden für intrinsische Motivation (IP2 $\left.{ }^{649} ; \mathrm{IP}^{650} ; \mathrm{IPF}^{651}\right)$. 
Befragte benennen diverse motivationale Aspekte für sozialunternehmerisches Handeln. Demnach können persönliche Betroffenheit in Bezug auf soziale Missstände ebenso intrinsisch motivierend wirken und zu sozialunternehmerischen Aktivitäten führen (IPن̈̈ ${ }^{652}$; IPE ${ }^{653} ; \mathrm{IPm}^{654} ; \mathrm{IP} 21^{655}$ ) wie persönliche Erfahrungswerte, die emotional stark berühren, so dass ein äußerer Anlass zum inneren Antreiber wird $\left(\mathrm{IPy}^{656}\right.$; IP $3^{657}$ ). Darüber hinaus wird geschildert, dass eine Begeisterung für den sozialunternehmerischen Ansatz ohne konkrete inhaltliche Zielsetzung bestand (IP28 ${ }^{658}$; IP $\tau^{659}$; IP $\beta^{660}$ ) und dass sich ein Thema z. B. im Rahmen der Reflexion über die eigene berufliche Zukunft erstmalig gezeigt hat $\left(\mathrm{IPa}^{661}\right.$; IP23 ${ }^{662}$, i. V. m. IP23 ${ }^{663}$; IP1 ${ }^{664}$, i. V. m. IP1 ${ }^{665}$ ). Eventuell entwickelt sich das sozialunternehmerische Interesse erst während eines Umsetzungsprozesses, wobei Offenheit gegenüber neuen Herausforderungen eine bedeutende Eigenschaft darstellen kann $\left(\mathrm{IP} \gamma^{666}\right)$. Ebenfalls kann beispielsweise die Motivation für unternehmerisches Denken mit der Entwicklung der sozialen Idee entstehen und ausreifen, ohne dabei erzwungen zu werden $\left(\operatorname{IPr}^{667}\right)$. Grundsätzlich ist jedoch zu bedenken, dass nicht jede soziale Idee eine unternehmerische Basis aufweisen muss (IP $\varepsilon^{668}$; IP18 ${ }^{669}$ ); z. B. im Hinblick auf den fehlenden Absatzmarkt bei einem Nischenprodukt (IP1 ${ }^{670}$, i. V. m. IP1 ${ }^{671}$ ).

Es ist davon auszugehen, dass sowohl KE als auch SE das Dilemma erleben, einerseits intrinsisch motiviert zu sein und sich andererseits mit der Marktrealität und dem Wirtschaftlichen kognitiv auseinanderzusetzen. Somit sollte ein Weg gefunden werden, der beiden eventuell im Widerspruch zueinanderstehenden Impulsen gerecht wird. Dabei ist weder in Naivität noch in Handlungsblockaden zu verfallen (IP17 772 ).

IP1 erklärt seine Demotivation zum entscheidungsrelevanten Bestandteil seiner Grundmotivation (IP1 ${ }^{673}$ ) und somit, sich von einem Mangelzustand entfernen zu wollen. Eine motivationale Zielsituation kann sich durchaus aus einer demotivierten Ausgangssituation heraus entwickeln (IP32 $2^{674}$; IPy ${ }^{675}$; IPR ${ }^{676}$; IPÜ ${ }^{677}$; $\operatorname{IP} \beta^{678}$; IP $\varepsilon^{679}$ ), also ein Weg-von-Zustand in ein Hin-zu-Ziel transformiert werden.

Unabhängig davon, inwieweit sozialunternehmerisches Engagement proaktiv oder reaktiv erfolgt und welche Beweggründe dem zugrunde liegen, prägten die von den Befragten benannten motivationalen Ausgangssituationen den eigenen beruflichen Karriereweg, der in Einklang zu Lebens-Werten gebracht wird (vgl. Schwartz, 1992; 2003; 2011). Dabei bewerten Befragte die inhaltliche Arbeit als emotional erfüllend und bezeichnen sie als Lebenswerk (IP9 ${ }^{680}$ ); dies bei bestehender innerer Überzeugung, dass gesellschaftlicher Mehrwert ermöglicht wird, was soziale Zielsetzung zur Herzenssache macht $\left(\mathrm{IPk}^{681}\right.$; IPÜ ${ }^{682}$; 
IP30 ${ }^{683}$ ). In der Gesamtbetrachtung zeigen alle Befragten im Hinblick auf ihr sozialunternehmerisches Engagement auf, erfolgsmotiviert zu sein.

Wird der innere Handlungs- und Verhaltensmotor angetrieben durch den Impuls, einen sozialen Missstand zu bearbeiten, kann dies mit einem Problemlösungsanspruch gekoppelt sein (IP18 ${ }^{684}$ ), muss es aber nicht (IP21 ${ }^{685}$ ). Dabei kann, wie in Abschnitt 7.3 im Rahmen der Diskussion zur Messung von Social Impact erörtert, selbst nach dem Verfehlen eines konkreten Ziels in anderer Weise Wirkung erzielt werden $\left(\mathrm{IPQ}^{686}\right)$, unabhängig davon, ob zuvor eine Wirkungskette formuliert worden ist. Da positive Wirkungsmessergebnisse motivierend wirken können, erscheint es ratsam, jede Form von Social Impact aufzudecken.

Die Basis für die personenbezogene Handlungsbereitschaft bildet ein individuell ausgestalteter Komplex an Aspekten, der u. a. aus den Fähigkeiten und Fertigkeiten besteht, die dem eigenen Können entsprechen (IP4 ${ }^{687} ; \mathrm{IPQ}^{688} ; \mathrm{IPw}^{689}$ ). Während Fähigkeiten (Abilities) zeitlich überdauernde Eigenschaften darstellen, wie z. B. ein vorhandenes Zahlen- und Rechenverständnis, spiegeln Fertigkeiten (Skills) sich verändernde, erworbene Kenntnisse wider, beispielsweise infolge eines absolvierten Trainings zur Bewältigung der betriebswirtschaftlichen Kostenrechnung im Rahmen der eigenen sozialunternehmerischen Aktivitäten. Dabei investieren Befragte zur eigenen Kompetenzerweiterung z. B. in die Teilnahme an einem SEO-U-Programm und in das Absolvieren eines speziellen Studiengangs $\left(\right.$ IP1 $9^{690}$, i. V. m. IP19 ${ }^{691}$ ). Die damit beabsichtigte Kompetenzerweiterung stellt für sich betrachtet eine Form der Leistungsmotivation dar.

\subsection{Motive und Phasen des Motivationsprozesses}

Die Prozessdynamik von Motivation lässt sich als aufeinander aufbauende Phasen darstellen. Heckhausen und Gollwitzer (1987; ausführlicher bei Heckhausen, Gollwitzer \& Weinert, 1987) beschreiben dabei in ihrem vierstufigen Handlungsphasenmodell folgende Abfolge: (1) eine initiale motivationale Bewusstseinsphase, (2) eine volitional geprägte präaktionale Phase, (3) eine volitional geprägte aktionale Phase und (4) eine postaktionale motivationale Bewusstseinsphase:

1. In der ersten Phase werden mögliche Handlungsalternativen abgewogen. Diese initiale motivationale Bewusstseinsphase passiert somit realitätsorientiert $\left(\right.$ IP14 $\left.{ }^{692}\right)$. Unter Umständen erlebt die betreffende Person im Hinblick auf ihr Handlungsvorhaben eine Qual der Wahl, so dass ggf. vom Aktivieren eines 
Kontrollprozesses mit Fazit-Tendenz ausgegangen wird. Ein damit verbundener Reflexionsprozess passiert prädezisional (IP11 ${ }^{693}$ ). Diese erste Phase mündet in die Intentionsbildung.

Die Intentionsbildung initiiert den darauffolgenden, volitionalen Motivationsanteil, der aus zwei realisierungsorientierten Phasen besteht:

2. In der ersten dieser beiden volitionalen Phasen werden präaktional Ziele gesetzt. Sie ist von selektiver Informationsaufnahme gekennzeichnet. Denn die Zielintention bedarf erst noch eines passenden Initiierungsmoments bzw. einer Umsetzungsgelegenheit. Dabei kann es zum Entstehen mehrerer Intentionen kommen, die miteinander in Konkurrenz treten. Diese werden nicht gleichzeitig aktiviert, sondern nur diejenigen, die Aufmerksamkeit auf sich ziehen. Diese zweite Motivationsphase mündet in die Handlungsinitiierung einer Zielintention, die sich gegenüber anderen konkurrierenden Zielintentionen durchgesetzt hat.

3. In der zweiten volitionalen Phase zeigt sich, dass mit dem Initiieren einer Handlung die Zielintention handlungsleitend wird: Aktionale Handlungen werden vorgenommen, bis das Handlungsergebnis erreicht ist. Eventuell kommt es zur Handlungsregulation (IP9 ${ }^{694}$ ).

Von der Volitionsstärke hängen Handlungsrichtung, Handlungsintensität und Handlungsausdauer ab (Achtziger \& Gollwitzer, 2010). Dabei stellt die Existenz eines Handlungsziels die Voraussetzung dafür dar, um im kognitiven Verarbeitungsprozess eine Handlungsrichtung einnehmen zu können. Volitional kann z. B. begünstigend wirken, wenn Hilfe und Unterstützung durch andere angenommen wird (IP4 $\left.{ }^{695}\right)$.

Zum Ende der zweiten Volitionsphase wird die vorgenommene Handlung beendet bzw. deaktiviert.

4. Die abschließende motivationale Bewusstseinsphase erfolgt postaktional. Dabei findet eine Bewertung der vollzogenen Handlung und der erzielten Handlungsergebnisse statt (IP10 $0^{696}$; IPE ${ }^{697}$; IPT ${ }^{698}$ ) und es lassen sich Lehren aus der Erfahrung für zukünftige Handlungen ziehen.

Im Übergang von der realitätsorientierten prädezisionalen Bewusstseinsphase zur realisierungsorientierten präaktionalen Volitionsphase vollzieht sich die Intentionsbildung für Handlungen (IP26 $6^{699}$; IP26 $6^{700}$; IP26 $6^{701}$ ). Die Autoren des Handlungsphasenmodells vergleichen diesen Phasenübergang als Entwicklungsschritt hin zu einem handlungsvorhabenbezogenen ,point of no return“ und erinnern dabei an Caesar und seine bewaffneten Truppen, die im Rahmen eines angebahnten Bürgerkriegs in Italien den Fluss Rubikon in Richtung Rom überquert 
hatten, was einer Kriegserklärung gegenüber dem römischen Senat gleichgekommen war und wobei Caesar bekundet haben soll, dass mit diesem Schritt sein (Entscheidungs-)Würfel gefallen sei. Infolge dieser Analogie wurde die beschriebene Handlungsphasen-Systematik als Rubikon-Modell bekannt.

Beim Rubikon-Modell wird davon ausgegangen, dass ein Handlungsverlauf davon abhängt, inwieweit es zu Beginn, also initial prädezisional, zur Stimulierung von Motiven kommt. Motive lassen sich als situationsübergreifend gültige Wertungsdispositionen einer Person beschreiben (Schneider \& Schmalt, 2000), die Einfluss darauf nehmen, worauf die jeweilige Person ihre Aufmerksamkeit lenkt, wie sie eine bestimmte Situation wahrnimmt und bewertet. Dabei stellen Leistung (IP9 ${ }^{702}$ ), sozialer Anschluss (IP $\left.\varphi^{703} ; \operatorname{IP} \pi^{704}\right)$ und Macht $\left(\operatorname{IPg}^{705}\right)$ fundamental bedeutsame Motive dar (z. B. ebd.; Nerdinger, 2013, S. 23 ff.). Darüber hinaus lassen sich Differenzierungen zwischen und Abgrenzungen zu weiteren Motiven vornehmen. Beispielsweise ordnet IP2 das Geldverdienen der sozialen Zielsetzung und dementsprechend Beziehungsmotiven unter, so dass unternehmerisches Leistungsverhalten für ihn kein Leitmotiv für sein sozialunternehmerisches Handeln abbildet (IP2 ${ }^{706}$ ). Eine weitere Differenzierung besteht zwischen Beziehungsmotiven, beispielsweise im Hinblick auf das Anerkennungsund das Wichtigkeitsmotiv (vgl. Sachse, 2020). Dabei wird motivübergreifend aufgezeigt, dass Handlungsausführungen durch ein soziales Dürfen beeinflusst werden (ebd.; Nerdinger, 2013, S. 7).

Eine Handlungsausführung kann dadurch begünstigt werden, wenn eine Situation ermöglicht bzw. wenn eine Person in einer Situation erwartet, motivbezogene Ziele erreichen zu können. Jedoch kann ein ausgeprägter Sättigungsgrad eines Motivs, einhergehend mit empfundener Motivbefriedigung, die Bereitschaft senken, eine Handlung fortzusetzen oder sie zu vollziehen - dies in Abhängigkeit von der Intensität, in der ein Motiv besteht und dementsprechend mehr oder weniger zur Befriedigung drängt.

Aus unterschiedlichen Gründen können verschiedenartige Ereignisse und Einflüsse zu Motivkonflikten führen, z. B. wenn ein und dasselbe Motiv für verschiedene Handlungsvorhaben bedeutsam erscheint. Beispielsweise gelang es IP18 oftmals nicht, sich im Hinblick auf sein Motiv, der Welt Unterstützung zu bieten, für eine der sich dabei reizüberflutend ergebenden Handlungsalternativen $\mathrm{zu}$ entscheiden (IP18 ${ }^{707}$ ). Ebenso können mehrere parallel ablaufende Lebensereignisse sich widersprechende Motive abverlangen. Korber berichtet beispielsweise bei einem der von ihr befragten SE einen Entscheidungskonflikt, bei dem der Betroffene zu entscheiden hatte, ,entweder mit der Partnerin und seinem Kind zusammenzubleiben oder seinem tiefen sozialen Anliegen für die vielen Armen zu kämpfen. Er traf die schwere Entscheidung für die Armen, obwohl er 
zu der Zeit damit noch nicht erfolgreich war“" (Korber, 2015, S. 124). Zudem können im Widerspruch zueinanderstehende oder unklare Vorgaben aus dem Umfeld vorliegen und ebenfalls Konflikte mit sich bringen (IP28 ${ }^{708}$ ). In der eigenen Biografie stark fehlbefriedigte Motive können sogar zu Persönlichkeitsstörungen führen (Sachse, 2020).

Zwar lassen sich Motive personenbezogen innerhalb einer entsprechend individuell ausgestalteten Landschaft zur Handlungsmotivation verorten. Allerdings unterliegen dabei Aussagen stets der Interpretation, welches Motiv in welcher Intensität als handlungsmotivierend bzw. -initiierend gilt. Denn Motive lassen sich im komplexen wie dynamischen Bedingungsgefüge menschlicher Handlungen weder eindeutig priorisieren noch lässt sich präzise und mit einem einheitlichen Verständnis bestimmen, welche Indikatoren letztendlich dem einen oder doch besser einem anderen Motiv zuzuordnen sind. Als nicht beobachtbare, hypothetische Konstrukte können Motive ausschließlich theoretisch analysiert und klassifiziert werden. Dabei kann eine Vielschichtigkeit an Beweggründen für Verhaltensweisen vorliegen, so dass ein personenbezogener Cocktail an Motiven, getrübt durch die Vielfalt seiner Bestandteile in unterschiedlicher Lagebeziehung zueinander, situativ Wirkung entfaltet (vgl. z. B. Nerdinger, 2013; Kelly, 1965; Murray, 1938; Maslow, 1971), was kausale Zuschreibungen dementsprechend behindert bzw. verbietet. Zudem wird im Rubikon-Modell einseitig auf bewusste Motive geachtet. Unbewusste Prozesse bleiben ebenso unberücksichtigt wie die Abgrenzung zu bzw. Berücksichtigung von Bedürfnissen, obwohl Bedürfnissen eine ausgesprochen hohe Relevanz im Motivationsprozess zukommt. Daher wird deren Bedeutung nun herausgearbeitet.

\subsection{Stellenwert von Bedürfnissen und Werten im Motivationsprozess}

In der Fachliteratur besteht weder zum Motivbegriff noch zum Bedürfnisbegriff ein einheitliches Verständnis: Mal wird der Motivbegriff dem Bedürfnisbegriff untergeordnet (z. B. Blickle, 2019a, S. 219), mal ist das Gegenteil der Fall (z. B. Nerdinger, 2019, S. 473). Wenngleich Maslow den Motivbegriff nur marginal nutzt (1971, S. 289 ff., 301; 1943/1999, Absatz 13, 67, 78), nimmt er ebenfalls keine eindeutige Abgrenzung zu Bedürfnissen vor (ebd.), sondern verwendet beide Begriffe zuweilen synonym (z. B. 1962/2014, Absatz 86, 104). Autorenübergreifend wird dabei nicht hinreichend reflektiert, welche spezifische, motivational wichtige Bedeutung bewusste Bedürfnisse bzw. Motive einerseits 
und unbewusste Bedürfnisse bzw. Motive andererseits haben (bei Maslow siehe ebenfalls 1971, S. 262).

Unter Berücksichtigung der bisherigen Ausführungen zum Verständnis von Motiven erscheint das Verständnis von Bedürfnissen grundsätzlich passend als „die Diskrepanz zwischen einem Sollwert und einem Istwert“ (Storch \& Krause, 2014, S. 88, m. V. a. Storch \& Kuhl, 2013, S. 90), wobei sich ein Bedürfnis durch ein Defizit mindestens eines Umweltelements bemerkbar macht. Ein als Spannung empfundenes Defizit kann einerseits physiologischer Natur sein und sich z. B. infolge eines Schlafdefizits oder Nahrungsmangels in Form von Müdigkeit bzw. Hunger als Bedürfnis bemerkbar machen; andererseits kann ein Defizit psychologischer Natur sein und sich z. B. bedingt durch fehlende Zuneigung oder einen Mangel an Erfolgserlebnissen als Bedürfnis zeigen. Im Umkehrschluss bedeutet dies nicht, dass ein befriedigtes Bedürfnis zwangsläufig an Bedeutung verliert. Beispielsweise bleibt das Bedürfnis, geliebt zu werden, bestehen, wenn es im Hier und Jetzt befriedigt wird. Im sprichwörtlichen Sinn kann z. B. das Liebesbedürfnis dem Fass ohne Boden gleichen. So kann eine Soll-Ist-Diskrepanz unterschiedlich schnell wieder zum Ausdruck kommen.

Ebenso kann ein ausgeprägter Sättigungsgrad eines Bedürfnisses bzw. eine empfundene Bedürfnisbefriedigung die Handlungsbereitschaft senken bzw. verhindern, vergleichbar zu Motiven. Dies hängt ebenfalls ab von der Intensität, in der ein Bedürfnis mehr oder weniger zur Befriedigung drängt. Allerdings setzen sich Motivationstheorien und -modelle, die ihren zentralen Beitrag zur bedürfnisorientierten Erklärung von Handlungsaktivierung leisten, in erster Linie mit Handlungsantreibern auseinander, die Push-Wirkungen zeigen; ein Beispiel hierfür ist die weiter unten in diesem Kapitel aufgezeigte Weiterentwicklung des Rubikon-Modells von Krause und Storch (2010, ausführlich dargestellt bei Storch \& Krause, 2014). Hingegen ermöglichen Motivationstheorien und -modelle, die ihren zentralen Beitrag zum Stellenwert von Motiven leisten, einen auf Anreize bezogenen Erkenntnisgewinn, die Pull-Wirkungen verkörpern, so wie es das ursprüngliche Rubikon-Modell repräsentiert. Insbesondere psychologische Bedürfnisse können unabhängig von einem Bewusstseinsgrad wirken.

Bereits Grawe (1998, S. 71) macht darauf aufmerksam, dass im RubikonModell unreflektiert bleibt, wie bewusst gewordene Handlungsmotive zustande kommen. Roth und Dicke (2005) zeigen hierzu passend aus neurowissenschaftlicher Perspektive auf, dass das limbische System maßgeblich am unbewusst ablaufenden Entstehen von Bedürfnissen und Gefühlen beteiligt ist und dass emotionale Zustände dabei nicht bzw. nur sehr eingeschränkt bewusst wahrgenommen werden können (vgl. auch Roth, 2003). Emotionale Zustände können daher auf unbewusstem Weg ihre Wirkung entfalten und dabei Einfluss auf 
Entscheidungsfindungsprozesse nehmen, dies sogar dominant, also handlungsentscheidend (ebd.; Roth \& Dicke, 2005; LeDoux, 2000). Dabei belegen funktionelle Kernspintomografie-Untersuchungen und weitere bildgebende diagnostische Verfahren, dass Reizleitungsprozesse des limbischen Systems deutlich kürzere Wege im neuronalen Netz zurücklegen und dadurch wesentlich schneller ablaufen als kognitive Prozesse. Dies verschafft emotionalen Aspekten in Entscheidungssituationen einen Vorteil bei der Bewertung von Bedürfnissen und spiegelt sich daraufhin im gezeigten Verhalten wider, sozusagen als neuronal geprägte Vor-Urteilsbildung (ebd.; vgl. auch Roth, 2019).

Storch und Krause (2014) integrieren diese Erkenntnisse in das RubikonModell und zeigen mit ihrer Modellerweiterung, die vor allem auf Basis neurowissenschaftlicher Erkenntnisse um eine vorgeschaltete Bedürfnis-Phase ergänzt ist, „dass ein bisher noch unbewusstes Bedürfnis die Umsetzung des bewussten Motivs verhindert. Meistens steht das unbewusste Bedürfnis im Gegensatz zum bewussten Motiv, sodass ein Motivkonflikt entsteht“ (S. 89). Somit kann die Push-Wirkung eines unbewussten Bedürfnisses die Pull-Wirkung eines bewussten Motivs überlagern.

Dieser Motivkonflikt zeigt sich in den Interviews bei SE, die auf kortikaler Ebene wissen, dass es richtig und wichtig ist, sich mit Finanzierungshürden bezüglich ihrer SEO jetzt auseinanderzusetzen, allerdings dennoch unter Aktivierung ihres limbischen Systems eine Aktivität zeigen, die sich besser anfühlt und der sozialen Mission entspricht (IPJ ${ }^{709}$; siehe auch Maxwell, Dunn, Rotz \& Shoji, 2019). Abbildung 8.1 zeigt diese Weiterentwicklung des RubikonModells, beispielhaft aufbereitet in Abbildung 8.2 zur Weiterbildungsmotivation eines Befragten, der sich ausschließlich auf die soziale Zielsetzung bezieht, nicht aber auf unternehmerische Themen und Fragestellungen (IPJ ${ }^{710}$ ).

Dass eine Emotionalisierung der sozialen Mission zulasten einer vernunftbasierten Bewertung unternehmerischer Aspekte geht, entspricht ebenso der in Abschnitt 3.4.2 dargestellten metaphernanalytischen Analyse zum fehlenden Konzeptansatz für die Verbindung des Sozialen und des Unternehmerischen. Im Hinblick auf die Beantwortung der zentralen Forschungsfrage erweist sich als bedeutsam, wie SE, die beispielsweise Widerstand gegenüber dem Unternehmerischen bekunden, zum unternehmerischen Denken und Handeln motiviert werden können. Das zugrunde liegende Dilemma lässt sich mit der Darstellung in Abbildung 8.3 im Vier-Felder-Kreuz zur Attraktivität bzw. Unattraktivität von Tätigkeiten und Ergebnissen veranschaulichen: Unattraktive Tätigkeiten können vermieden werden, selbst wenn das zu erwartende Ergebnis attraktiv ist. Die akute Bedürfnisbefriedigung setzt sich in diesem Fall zulasten des attraktiven Ergebnisses durch. 
Maslow führte biografische Analysen durch zur Erforschung von Bedürfnissen (1971; zum Werdegang vgl. 1943/1999; 1943a; 1954; 1962/2014). Die dabei identifizierten Bedürfnisse bezeichnet er als Basisbedürfnisse, die er wiederum in Defizit- und in Wachstumsbedürfnisse untergliedert. In der Fachliteratur wird bis heute zumeist eine veraltete, fünfstufige Version mit Bedürfniskategorien dargestellt, dies zudem als eine Pyramidenstruktur, die nicht von Maslow stammt und die sich zudem als unpassend erweist, da sie fälschlicherweise eine absolut erreichbare Bedürfnisbefriedigung an der Pyramidenspitze suggeriert. Stattdessen entwickelte Maslow eine deutlich komplexere Bedürfnisstruktur-Systematik und überarbeitete im Laufe seiner Forschungsarbeiten diverse Aussagen aus der somit obsolet gewordenen Erstdarstellung.
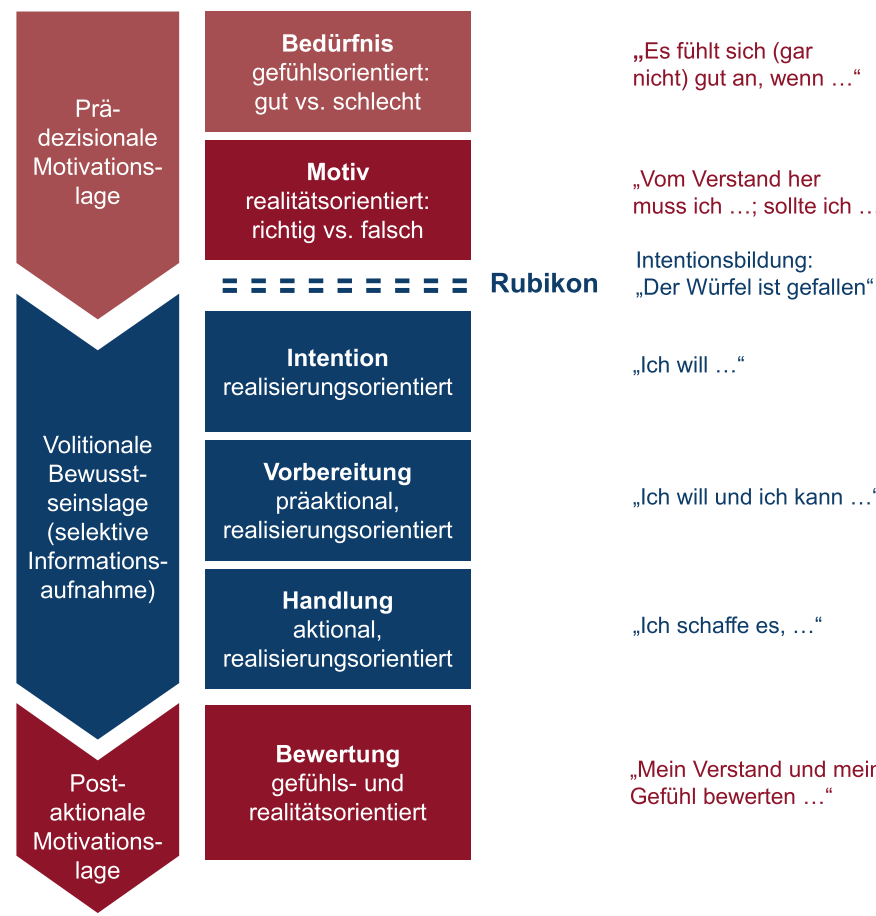

„Vom Verstand her muss ich ...; sollte ich ..."

Intentionsbildung:

„Der Würfel ist gefallen“

Intention

realisierungsorientiert

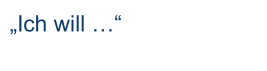

Vorbereitung

präaktional,

realisierungsorientiert

Handlung

aktional,

realisierungsorientiert

„Ich schaffe es, ..."
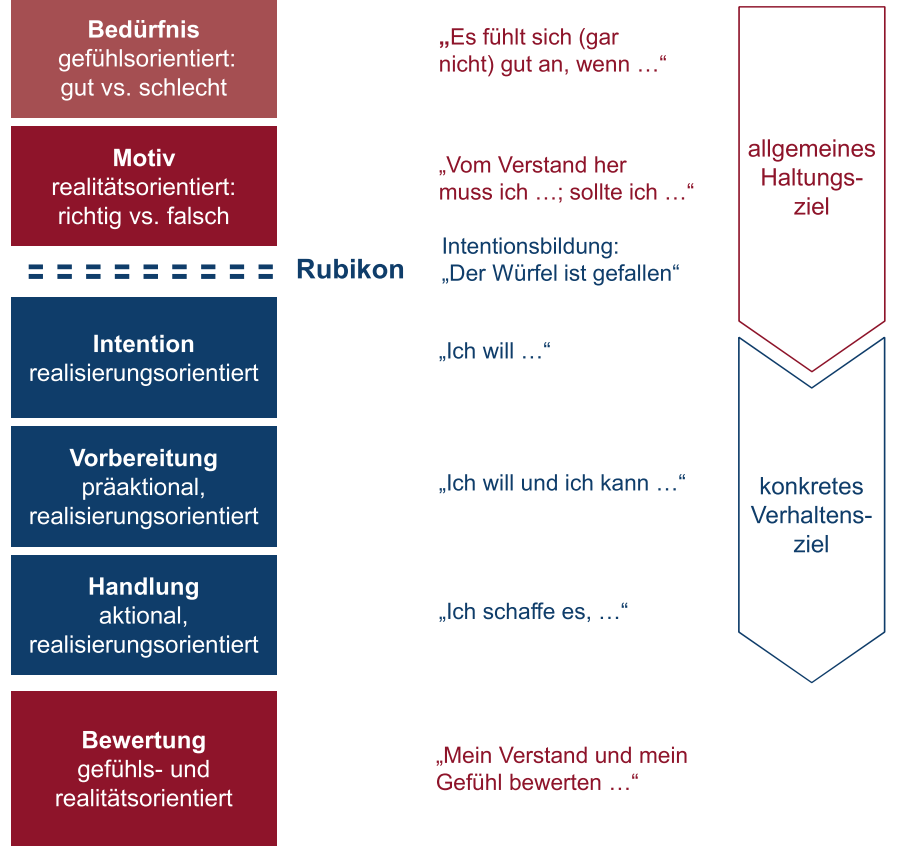

„Ich will und ich kann ..."

konkretes

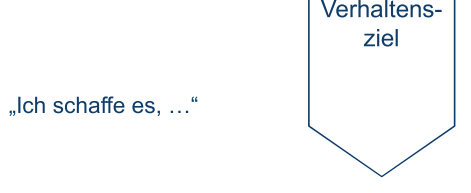

„Mein Verstand und mein

Gefühl bewerten ..."

Abbildung 8.1 Erweitertes Rubikon-Modell. (eigene Darstellung, in Anlehnung an Storch \& Krause, 2014, S. 87) 


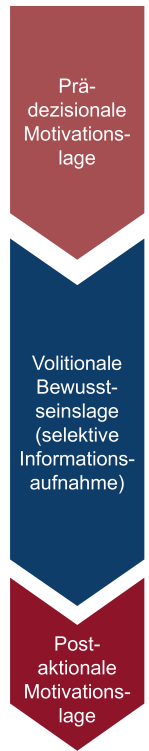

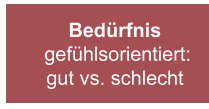

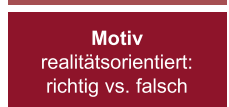

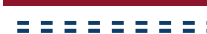

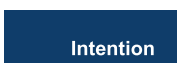

realisierungsorientiert

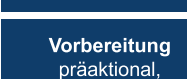

präaktional,

realisierungsorientiert

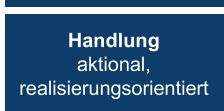

Bewertung

gefühls- und

realitätsorientiert
„MEINE persönliche Qualifizierung (.) war mir immer WICHTIG, (...) ich (...) habe mich mehr von meinen Interessen so leiten lassen"

"Aber nicht so im Sinne von, ich muss mich jetzt noch mehr qualifizieren"

„Unterschiedliche (Aus- und Weiterbildungen) [haben] mich jeweils angesprochen"

„SPANNEND! Und wo ich dann noch weitere Seminare mache oder weitere Ausbildungen mache. (.) Und das kommt letztlich eben, ne, den (Wirkungsempfängern), meinen KLIENTEN zugute, aber mir persönlich auch"

["Ich] habe im Laufe der Jahre vielerlei [Aus- und Weiterbildungen] durchlaufen"

"[Ausbildungsinhalte habe ich] ZUM TEIL am Ende wieder verworfen $(. .$.$) und gedacht (. .$.$) : Hm, nein,$ da ist jetzt doch nicht meins. Ich habe mir aus allen dieser Ausbildungen einzelne Aspekte rausgepickt, wo ich, (.) also mit denen ich mich KONFORM GEFÜHLT HABE und das weggelassen, was mir nicht (.) so in, (.) mir persönlich so NAHE war"

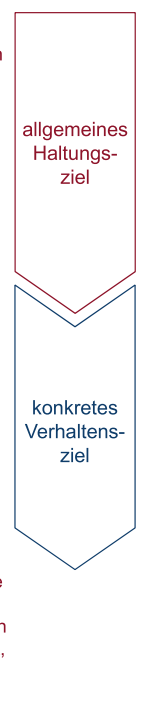

Abbildung 8.2 Erweitertes Rubikon-Modell, illustriert am Beispiel der Weiterbildungsmotivation von IPJ. (eigene Darstellung)

\begin{tabular}{|c|c|c|}
\hline & $\begin{array}{l}\text { Ergebnis } \\
\text { unattraktiv }\end{array}$ & $\begin{array}{r}\text { Ergebnis } \\
\text { attraktiv }\end{array}$ \\
\hline 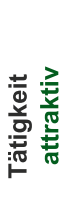 & $\begin{array}{l}\text { z. B. anderen voller Stolz das sozi- } \\
\text { alunternehmerische Konzept prä- } \\
\text { sentieren, das sodann von ande- } \\
\text { ren geklaut wird (z. B. IPN) } \\
\text { z. B. Wirkungsziele setzen, die } \\
\text { nicht erreichbar sind (z. B. IP17) }\end{array}$ & $\begin{array}{l}\text { z. B. in eigener SEO inhaltlich tätig } \\
\text { sein und soziale Wirkung bei Wir- } \\
\text { kungsempfänger erleben (z. B. IP2) } \\
\text { z. B. mit anderen SEO kooperieren } \\
\text { und darüber höheren sozialen Im- } \\
\text { pact zu generieren (z. B. IP6) }\end{array}$ \\
\hline 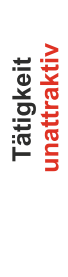 & $\begin{array}{l}\text { z. B. mit Politiker auseinander- } \\
\text { setzen, der Aussagen für seine } \\
\text { politischen Interessen instrumen- } \\
\text { talisiert (z. B. IP33) } \\
\text { z. B. mit potenziellem Investor } \\
\text { auseinandersetzen und dabei } \\
\text { zusätzlich verschulden, worauf- } \\
\text { hin der Investor sein Wort } \\
\text { dennoch nicht hält (z. B. IP1) }\end{array}$ & $\begin{array}{l}\text { z. B. Businessplan ausarbeiten, } \\
\text { sich mit betriebswirtschaftlichen } \\
\text { Fragestellungen auseinanderset- } \\
\text { zen, was eine Finanzierungs- } \\
\text { zusage zur Folge hat (z. B. IP26) } \\
\text { z. B. Mitarbeiter nach massivem } \\
\text { Fehlverhalten kündigen, um sozi- } \\
\text { aler Zielsetzung treu zu bleiben } \\
\text { (z. B. IP24) }\end{array}$ \\
\hline
\end{tabular}

Abbildung 8.3 Tätigkeits-Ergebnis-Attraktivität im Vier-Felder-Kreuz mit IP-Beispielen. (eigene Darstellung, angelehnt an Storch \& Krause, 2014) 
Maslows Ausführungen basieren auf dem „,westlichen“, individualistisch geprägten Kulturverständnis und können daher für eine Auseinandersetzung mit dem Sozialunternehmertum in Deutschland herangezogen werden. Jedoch ist zu berücksichtigen, dass er ein empirisch nicht gut überprüfbares Erklärungsmodell entwickelt hat, zumal er mit seinem klinisch-psychologischen Hintergrund keinen Bezug zum Unternehmertum aufweist. Dennoch erweist sich das Heranziehen seines Bedürfniskonzepts im Rahmen dieser Arbeit als aufschlussreich.

In der erweiterten, aktualisierten Darstellung unterscheidet Maslow bei den Defizitbedürfnissen folgende Kategorien:

- physiologische Bedürfnisse, z. B. nach Schlaf und Ernährung (IPÜ ${ }^{711}$ ),

- Sicherheitsbedürfnisse, z. B. nach einem gesicherten Arbeitsverhältnis und nach Altersabsicherung $\left(\mathrm{IP}^{712} ; \mathrm{IPU}^{713} ; \mathrm{IPi}^{714}\right)$,

- soziale Bedürfnisse, z. B. nach Gruppenzugehörigkeit und Kontaktgestaltung $\left(\mathrm{IP} \pi^{715}\right)$,

- Ego-Bedürfnisse (Individualbedürfnisse), z. B. nach Anerkennung, Status und Prestige (IP34 ${ }^{716}$ ),

- kognitive Bedürfnisse, z. B. das Bedürfnis nach Wissen und das Bewusstsein über die Bedeutung von Werten (IP19 $9^{717}$ ), und

- ästhetische Bedürfnisse, z. B. das Bedürfnis nach Schönheit und nach Ordnung ( $\left.\mathrm{IPx}^{718}\right)$.

Allerdings diskutiert Maslow kognitive und ästhetische Bedürfnisse ebenso als Wachstumsbedürfnisse; eine unmissverständliche Trennschärfe wird hier nicht immer deutlich (vgl. z. B. Maslow, 1962/2014, Absatz 92). Als Wachstumsbedürfnisse benennt Maslow eindeutig das unstillbare Selbst-Aktualisierungsbedürfnis und das ebenso unstillbare Transzendenzbedürfnis.

An dieser Stelle wird die von Maslow gewählte Bezeichnung ,SelfActualization" beibehalten und darauf aufmerksam gemacht, dass die oft gebrauchte deutsche Übersetzung ,Selbstverwirklichung“ zur Verwässerung einer Kernaussage Maslows führt. Denn "Self-actualization is an ongoing process“ (Maslow, 1971, S. 44), ,not only an end state but also the process of actualizing one's potentialities at any time, in any amount“ (ebd., S. 46); dementsprechend „not a matter of one great moment. Self-actualization is a matter of degree, or little accessions accumulated one by one" (ebd.). In Abgrenzung hierzu kann Selbstverwirklichung irrtümlicherweise als ein Zustand verstanden werden, der absolute Erreichbarkeit ermöglicht. In Analogie zu dem Computer, an dem diese 
Arbeit geschrieben wird, ist das kontinuierliche Aktualisieren der genutzten Software ein gewünschter Prozess, um in der Interaktion mit anderen mit der Zeit zu gehen und auf dem neusten Stand der Technik zu sein, also Kontakt und Austausch zu anderen zu ermöglichen. Selbst-Aktualisierungsprozesse lassen sich dabei eventuell als Meilensteine eines Entwicklungswegs definieren. Eine Selbstverwirklichung des Computers hingegen wäre unerwünscht, wenn sie eine Form von Eigensinn verkörpert, die einen endlichen Zustand repräsentiert und keineswegs Kontakt zur Umwelt gewährleisten muss. Zudem erscheint die inhaltliche Übersetzung von Selbstverwirklichung mit ,Self-realization“ angebrachter. Selfactualization hingegen, is the merging of subject and object, involving no loss of subjectivity but what seems is infinitive extension” (Maslow, 1971, XVII), ,,means experiencing fully, vividly, selflessly, with full concentration and total absorbtion (...), the person is wholly and fully human. This is a self-actualizing moment. (...) Self-actualization implies that here is a self to be actualized (...), letting the self emerge“ (Maslow, 1971, S. 44).

Unter Selbstverwirklichung ist hierbei eben nicht zu verstehen, sich selbst zu verwirklichen und dabei nur eigene Interessen zu verfolgen. Denn nach Maslow ist das eigene Selbst in einem kontinuierlichen Aktualisierungsprozess zu verwirklichen, was im Kontakt zur Umwelt passiert. „Each time one takes responsibility, this is an actualizing of the self" (Maslow, 1971, S. 45). Zugrunde liegt ein Selbst-Verständnis sozialer Natur: ,Self-actualizing people ...

- manage somehow simultaneously to love the world as it is and to try to improve it. In all cases there was some hope that people and nature and society could be improved“ (Maslow, 1971, S. 298) (IP14 ${ }^{719}$, i. V. m. IP14 ${ }^{720}$ );

- tend to feel that every person should have an opportunity to develop to his highest potential, to have a fair chance, to have equal opportunity. One advantage of being a boss is the right to give away the corporation's money, to choose which good causes to help. They enjoy giving their own money away to causes they consider important, good, worthwhile, etc. Pleasure in philanthropy“ (Maslow, 1971, S. 299) (IP10 ${ }^{721}$ );

- are described as those who listen to their own voices, take responsibility“ (Maslow, 1943a) (IP8 ${ }^{722}$ );

- enjoy taking on responsibilities (that they can handle well), and certainly don't fear or evade their responsibilities. They respond to responsibility“ (Maslow, 1971, S. 299) (IP6 $\left.{ }^{723}\right)$;

- enjoy doing good“ (Maslow, 1971, S. 298) (IPJ ${ }^{724}$ );

- delight in bringing about justice“ (ebd.) (IP21 ${ }^{725}$ ); 
- hate (and fight) corruption, cruelty, nalice, dishonesty, pompousness, phoniness, and faking“" (Maslow, 1971, S. 299) (IPI ${ }^{726}$ );

- try to set things right, to clean up bad situations“" (Maslow, 1971, S. 298) $\left(\mathrm{IP} 33^{727}\right)$;

- uniformly consider their work to be worthwhile, important, even essential“" (Maslow, 1971, S. 299) (IP $\psi^{728}$ );

- are dedicated people, devoted to some task ,outside themselves', some vocation, or duty, or beloved job. Generally the devotion and dedication is so marked that one can fairly use the old words vocation, calling, or mission to describe their passionate, selfless, and profound feeling for their ,work ““ (Maslow, 1971, S. 291) (IP2 ${ }^{729}$ );

- learn through the processes of intrinsic learning“ (Maslow, 1943a) (IPä $\left.{ }^{730}\right)$;

- are not primarily motivated (i. e. by basic needs); they are primarily metamotivated (i. e. by metaneeds = being values)“ (Maslow, 1971, S. 301). „The best way to communicate these feelings to someone who doesn't intuitively, directly understand them is to use as a model ,falling in love " (Maslow, 1971, S. 293) (IP23 $\left.{ }^{731}\right)$;

- enjoy greater efficiency, making an operation more neat, compact, simpler, faster, less expensive, turning out a better product, doing with less parts, a smaller number of operations, less clumsiness, less effort, more foolproof, safer, more ,elegant", less laborious“" (Maslow, 1971, S. 299) $\left(\mathrm{IPy}^{732}\right)$.

Wie bereits angedeutet, geben Befragte Ereignisse an, die zur Auseinandersetzung mit sozialunternehmerischen Gedanken führten. Diese Ereignisse stellen eine Auseinandersetzung mit der eigenen Verantwortungsübernahme und einen Aktualisierungsprozess des Selbst dar. Beispiele sind das Lesen von Büchern bzw. Verinnerlichen von Gedanken anderer ( $\mathrm{IPq}^{733}$; IP19 ${ }^{734}$; IP30 ${ }^{735} ; \mathrm{IP}^{736}$; IP23 ${ }^{737} ; \operatorname{IPr}^{738}$; IPp ${ }^{739}$; IP $\ddot{A}^{740}$; IP33 $3^{741}$ ), die Auseinandersetzung mit Vorbildfiguren (IP23 ${ }^{742}$; IP23 $3^{743}$; IP2 $8^{744}$ ), bemerkenswerte Lebensereignisse $\left(\operatorname{IPk}^{745}\right.$ ) und Schlüsselerlebnisse im persönlichen Werdegang, die sich in der SEO-Idee widerspiegeln $\left(\mathrm{IP}^{746}\right.$; IP28 ${ }^{747}$ ) und zu Veränderungen bis hin zu Brüchen im Sozialgefüge führen können (IP23 $3^{748}$ ).

Schließen Betroffene während ihres Selbst-Aktualisierungsprozesses mit einem Teil ihrer persönlichen Geschichte ab, brechen aus bisherigen Grenzen 
aus und erleben dadurch einen qualitativen Aufstieg (IP23 ${ }^{749}$; zur motivationalen Bedeutung von persönlichen und sozialen Bedürfnissen siehe auch Ambati, 2020), so entspricht dies einem Transzendieren, abgeleitet aus dem Lateinischen transcendere (hinübersteigen, überschreiten). Befragte reflektierten in ihrem Werdegang bewusst ihre Persönlichkeitsentwicklung, die sich aufgrund von grundlegenden Veränderungen in Wertigkeiten und deren Priorisierungen als harter Kampf mit sich selbst umschreiben lässt (IP28 ${ }^{750}$; IPÖ ${ }^{751}$ ). Von hoher Bedeutung ist für IP23 geworden, wie er zwischenmenschlichen Kontakt gestaltet und welche Kontaktgestaltung er sich von seinem Gegenüber wünscht. Sein eigener Haltungs- und Erwartungshaltungsanspruch ist von respekt- und würdevollem Umgang in Gegenseitigkeit geprägt (IP23 $3^{752}$, i. V. m. IP $23^{753}$, IP $23^{754}$, IP $23^{755}$, IP23 ${ }^{756}$ \& IP23 ${ }^{757}$ ). Dabei werden Erfahrungen zu Erlebnissen besonderer Güte. Diese „Peak experiences are transient moments of self-actualization“ (Maslow, 1971, S. 46). „A peak experience is what you feel and perhaps ,know“ when you gain authentic evaluation as a human being (...). It comes and it goes and it cannot be forgotten“ (Maslow, 1971, S. XVI). Peak experiences können nicht gesucht und willkürlich gefunden werden, sondern im übertragenen Sinn finden sie jemanden (IPE ${ }^{758}$; als Gegenbeispiel: $\mathrm{IPE}^{759}$ ). Maslow erkennt hierin einen Übergang von Selbst-Aktualisierung zur Transzendenz (1971, S. 3 f.).

Wie bereits in Kapitel 2 aufgezeigt, lassen sich bei Social Startups sowohl direkte negative Effekte nachweisen für ,Self-Enhancement“, das für das Verfolgen von Eigeninteressen steht, als auch deutlich positive Effekte für ,SelfTranscendence", bei der gemeinwohlorientierte Werte, das Wohlergehen der Gesamtheit sowie das Verfolgen von Gemeinschaftsinteressen handlungsleitend sind (vgl. Kruse, Wach, Costa \& Moriano, 2018). Menschen mit stark ausgeprägter Self-Transcendence genügen sich nicht selbst, sondern sind auf Reizaufnahme durch andere Menschen oder durch Ereignisse angewiesen (IP2 ${ }^{760}$; vgl. auch Pangriya, 2019). Als Voraussetzung für Self-Transcendence gelten Selbstdistanzierung ebenso wie Dialogbereitschaft (IPy ${ }^{761} ;$ IPÖ $^{762} ; \operatorname{IP}_{17}^{763}$ ).

Aus Maslows Transzendenz-Ansatz ging die sog. ,vierte Kraft“ der Psychologie hervor; als sog. „erste Kraft“ wird allgemein die psychoanalytische Ausrichtung bezeichnet, als sog. „zweite Kraft“ die naturwissenschaftlichbehavioristische Ausrichtung. Als Gegenentwurf zu diesen beiden „Kräften“ entwickelten u. a. Rogers (1961), Perls (Perls, Hefferline \& Goodman, 1951) und Maslow als sog. „dritte Kraft“ der Psychologie die humanistische Psychologie. Sie ist von der Auseinandersetzung mit Selbst-Aktualisierungstendenz geprägt. Die aus Maslows Transzendenz-Ansatz hervorgegangene ,vierte Kraft“ wird auch transpersonale Psychologie genannt und basiert auf der Auseinandersetzung mit Metabedürfnissen zu spirituellen Erfahrungen. Hierbei wird ein Ich-bezogenes 
Wohlbefinden zugunsten prosozialer Beziehungsgestaltung und Lebenserfüllung nicht nur gesucht, sondern auch gefunden (zum vertiefenden Verständnis und aktuellen Überblick der forces in psychology siehe Fleuridas \& Krafcik, 2019).

Dieser Metaebenen-Ansatz kann erreicht werden bei einer ,Transcendence of the basic needs (either by gratifying them so that they disappear normally from consciousness, or by being able to give up the gratifications and to conquer the needs). This is another way of saying, to become primarily metamotivated'. It implies identification with the B[eing]-Values“ (Maslow, 1971, S. 262). Unter „Being-values“, auch „,values of being“, versteht Maslow „the ultimative values which are intrinsic, which cannot be reduced to anything more ultimate" (ebd., S. 42).

Befragte SE vertrauen darauf, dass ihr Handeln sinnvoll ist (IP6 ${ }^{764}$ ), wobei ein hoher Stellenwert von Empathie deutlich wird (IP2 ${ }^{765}$; IP23 $3^{766}$; IP23 ${ }^{767}$; IP2 ${ }^{768}$; zum Bedeutungsgehalt von Empathie bei SE vgl. z. B. Ruskin, Seymour \& Webster, 2016; Lambrechts, Caniëls, Molderez, Venn \& Oorbeek, 2020). Dabei weisen die Attributionen Maslows hinsichtlich self-actualizing People zwar eine offensichtliche Überschneidung zu Schilderungen von Befragten auf. Allerdings sind ausgeprägte Selbst-Verwirklichung und Selbst-Aktualisierung auf metamotivationaler Ebene und ein Transzendenzbedürfnis keinesfalls gleichzusetzen mit Sozialunternehmertum. Bezogen auf sozialunternehmerisches Denken würde die einseitige Betrachtung der von Maslow benannten Eigenschaften eine Romantisierung darstellen, unternehmerische Anforderungen ausklammern und somit ein Klischeebild eines SE zeichnen, das Self-Actualization mit Sozialunternehmertum verwechselt, gleichsetzt bzw. zu einer Einheit vermischt. Ein Anspruchsdenken gegenüber SE, alle Wachstumsbedürfnisse optimal befriedigen zu können, würde ebenso absurd erscheinen wie die Vorstellung, dass Menschen, die ihre Wachstumsbedürfnisse optimal befriedigen, hiermit die wesentliche Basis für qualitativ hochwertige sozialunternehmerische Aktivitäten bilden. Vielmehr lässt sich mit den bisherigen Ausführungen eine grundsätzliche Problematik aufzeigen: dass ein Achtgeben auf die eigenen Bedürfnisse nicht nur bedeutet, gegen gesellschaftlichen Widerstand ankämpfen zu müssen, sondern Widerstand im Selbst erkennen und so auflösen zu können, dass sozialunternehmerisches Engagement dabei gezeigt und gelebt werden kann.

Wie weiter oben beschrieben, kann ein Individuum einerseits von seinen Bedürfnissen getrieben sein und andererseits von dem, was nach dem Befriedigen der Bedürfnisse entstehen soll, angezogen werden: der Motivbefriedigung. Diese Anziehungskraft spiegelt Wertvorstellungen wider. Da eine Kultur durch ihre Werte repräsentiert wird und da Werte von Kultur zu Kultur variieren, kann es dazu kommen, dass eine subjektiv erlebte Unvereinbarkeit von Sozialem und Unternehmertum als Kultur-Werte-Konflikt erlebt wird (IP14 ${ }^{769}$; IP $\varepsilon^{770}$ ). 
Hierbei kann sich der SE in einem Spannungsfeld befinden: Einerseits teilt er mit seinen Mitarbeitern und mit Netzwerkpartnern gemeinsame Werte, verkörpert als soziale Mission. Andererseits hat er eine unternehmerische Verantwortung, verkörpert beispielsweise als Abwägungsprozess bei der Verteilung knapper Ressourcen. Allerdings darf das soziale Wertesystem beim Generieren von Profit nicht zerstört werden (IP10 ${ }^{771}$ ).

Sieht der SE die soziale Zielsetzung als einen anzustrebenden Wert, ohne sich dabei mit dem Unternehmerischen zu identifizieren, dann identifiziert er sich demzufolge nicht mit seiner SEO, was zu motivationalen Einbußen führen kann. Dass Werte-Diskrepanzen zwischen Führungskräften und Unternehmen sich negativ auf das eigenverantwortliche Handeln der Führungskräfte und deren intrinsische Motivation auswirken, zeigen Jung und Morner (2016, m. V. a. Ryan \& Deci, 2000; Deci, Connell \& Ryan, 1989; Osterloh \& Frey, 2000). Im Rahmen ihrer Untersuchung zur Wirkweise von gemeinsamen Werten im Zusammenhang mit der Eigenverantwortung von Führungskräften gelangen die Autoren zu der Erkenntnis, ,dass gemeinsame Werte die Basis für Eigenverantwortung der Führungskräfte bilden und darüber hinaus einen recht hohen direkten Zusammenhang mit der intrinsischen Motivation aufweisen" (Jung \& Morner, 2016, S. 252). Zudem wirkt „Eigenverantwortung der Führungskräfte nicht nur direkt auf deren intrinsische Motivation, sondern auch als partieller Mediator auf den Zusammenhang zwischen gemeinsamen Werten und intrinsischer Motivation“ (ebd., S. 253), was im Falle eines Mangels zur Minderung der Arbeitsleistung führen kann (ebd.; vgl. auch Brieger, De Clercq \& Meynhardt, 2020). Dabei besitzen Ergebniskontrolle und Ergebnisbelohnung eine hohe Bedeutung für die Selbststeuerungsfähigkeit. Bereits Ouchi (1979) geht davon aus, dass sich Selbstbestimmung durch gemeinsame Werte fördern lässt.

Intrinsische Motivationseffekte lassen sich nicht einfach von Unternehmen zu Unternehmen imitieren (vgl. Coff \& Kryscynski, 2011). Denn: „Although the nature of values and their structure may be universal, individuals and groups differ substantially in the relative importance they attribute to the values. That is, individuals and groups have different value ,priorities" or ,hierarchies“" (Schwartz, 2012, S. 3). Allerdings können zur Stärkung intrinsischer Motivation in Teams und dementsprechend ggf. in SEO gemeinsame Werte genutzt werden und als Leitprinzipien einer sozialen Einheit dienen (vgl. Schwartz, 1994, S. 21). Quinn und Rohrbaugh (1983) entwickelten im Rahmen ihrer Untersuchungen zu Werten mit Einflussnahme auf organisationalen Erfolg ein Modell, das sich widersprechende bzw. konkurrierende Werte in Beziehung zueinander setzt: organisationsinterne versus organisationsexterne Fokussierung einerseits und formale versus flexible Strukturiertheit andererseits. Hierbei arbeiten die Autoren heraus, 
dass die Beurteilung der Effektivität einer Organisation letztendlich von der Klarheit über die Werte bzw. über deren Relevanz abhängt. Doch mangelt es gerade hierzu an geeigneten Vorschlägen. Übertragen auf die Situation der SE steht die Entwicklung einer Analyseform aus, die es ermöglicht, den Ist-Zustand einer SEO zu ihren sozialen Werten und zu ihren unternehmerischen Werten aufzuzeigen und dabei zu gewährleisten, sie als gleichberechtigte Wertequalitäten reflektieren und ggf. gewichten zu können sowie eine angemessene Verantwortungsübertragung auf Mitarbeiter zur Stabilisierung der intrinsischen Motivation aller Beteiligten zu ermöglichen. In Abschnitt 8.12 wird hierauf im Rahmen der Diskussion zu Unvereinbarkeiten zwischen der sozialen und der unternehmerischen Zielsetzung näher eingegangen.

Maslow hält an einer als widerlegt anzusehenden hierarchischen Ordnung fest, wenn er eine umfassende Befriedigung aller Defizitbedürfnisse als Grundvoraussetzung für das Befriedigen von Wachstumsbedürfnissen unterstellt (Maslow, 1971, S. 289; zur Widerlegung der Bedürfnishierarchie aus neuropsychologischer Sicht vgl. z. B. Roth \& Dicke, 2005; LeDoux, 2000; Storch \& Krause, 2014). Allerdings relativiert er eine Hierarchisierung für ein nicht befriedigtes Wachstumsbedürfnis. Denn hierbei beschreibt Maslow, dass die Grundbedürfnisse einer Person i. d. R. teilweise befriedigt und gleichzeitig teilweise unbefriedigt sind. Dabei ist der Sättigungsgrad eines Bedürfnisses von Person zu Person in unterschiedlicher Intensität ausgeprägt; ebenso verhält es sich innerhalb einer Person von Situation zu Situation (Maslow, 1943/1999, Absatz 61; 1971). Je nachdem, inwieweit ein Bedürfnis befriedigt oder beispielsweise unterdrückt ist, taucht es dementsprechend mehr oder weniger auf. Das hierarchische Verständnis ist somit dynamisch zu verstehen, wobei einige Bedürfnisse einen stabileren i. S. v. länger aufrechterhaltenden Zustand der Befriedigung aufweisen und andere Bedürfnisse stark volatil in ihrem Sättigungsgrad erscheinen können. Ebenso kann dabei mal das eine und mal das andere Bedürfnis an Bedeutung gewinnen oder verlieren, also eher in den Vordergrund bzw. in den Hintergrund rücken. Darüber hinaus beschreibt Maslow, dass Bedürfnisse in situativ bedingten Lagebeziehungen zueinanderstehen können. Beispielsweise kann das Transzendenzbedürfnis in unterschiedlicher Intensität mit dem Zustand der Selbst-Aktualisierung in Verbindung stehen, was im stark ausgeprägten Verbindungszustand die weiter oben bereits beschriebene Metamotivation widerspiegelt (1971, S. 259 ff., 289 ff.). In diesem Sinn kann z. B. eine ressourcenorientierte Lernkultur als ,lebenslanges Lernen" praktiziert werden, auf deren Basis die eigene Achtsamkeit kontinuierlich geschärft wird; dies schildert beispielsweise IPJ (IPJ ${ }^{772}$ ).

Im Grundverständnis wirken die einzelnen Basisbedürfnisse situativ bedingt mal mehr und mal weniger prominent und sie können sich gegenseitig überlagern, 

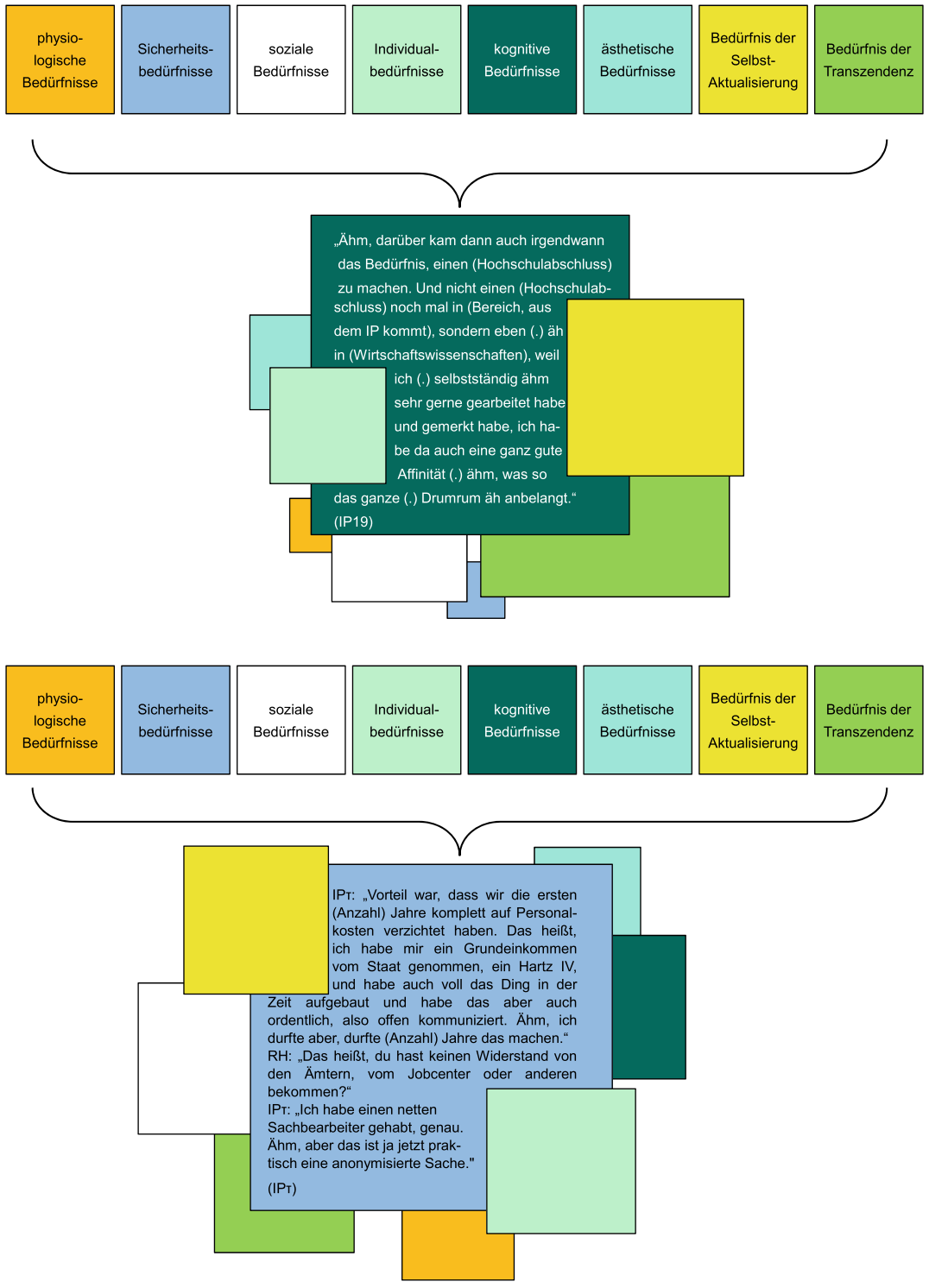

Abbildung 8.4 Basisbedürfnisse nach Maslow: zwei fiktive Anwendungsbeispiele. (eigene Darstellung) 
wobei anzustreben ist, dass Wachstumsbedürfnisse einen zentralen Stellenwert im Leben erlangen. Diese Dynamiken lassen sich mit der oftmals vorgefundenen Pyramidenstruktur nicht abbilden. Abbildung 8.4 stellt einen Versuch dar, sich dieser Herausforderung mit einem grafischen Neuentwurf zu stellen. Veranschaulicht wird dies mit zwei Beispielen. Wenngleich dabei Interviewzitate herangezogen werden, erfolgt dies jedoch als rein fiktive Darstellung und ohne Anspruch auf Passgenauigkeit für diese IP. Zudem kann die Abbildung nur Momentaufnahmen wiedergeben, so dass auch diese Darstellungsform an ihre Grenzen stößt, da die Prozessdynamik, der die Bedürfnispositionierungen unterliegen, dabei nicht zum Ausdruck kommen kann. Dennoch lässt sich das Grundverständnis Maslows abbilden.

Während sich Defizitbedürfnisse bei Mangelzuständen oder Störungen dem Prinzip der Homöostase unterwerfen und somit ein inneres Gleichgewicht anstreben, demzufolge ein Sättigungsgefühl erlebt werden kann, ist dies bei Wachstumsbedürfnissen nicht in dieser Form gegeben. Zudem kann die Befriedigung sowohl von Defizit- als auch von Wachstumsbedürfnissen das Ausbilden gesundheitsgefährdender Strukturen nur begrenzt vermeiden (ebd.), und wenngleich ,,a healthy man is primarily motivated by his needs to develop and actualize his fullest potentialities and capacities“ (Maslow, 1943/1999, Absatz 75), können befriedigte Wachstumsbedürfnisse psychologische Gesundheit begünstigen, keinesfalls jedoch garantieren.

Modelle zu Bedürfnissen und zur intrinsischen Motivation können somit nur einen typologiebasierten Erklärungsansatz für Problemsituationen im Nachhinein bieten, jedoch keinen wesentlichen Beitrag zur Vorhersage leisten. Dennoch weisen sie darauf hin, dass bestimmte Arbeitsbedingungen die Motivation und Demotivation beeinflussen können.

\subsection{Positionierung gegenüber dem Geldverdienen}

Befragte heben hervor, dass das Geldverdienen im Können und Dürfen eine Herausforderung darstellt (IP $\tau^{773}$; IP34 ${ }^{774}$ ) und nicht bedingungslos geschieht. Erwähnt wurde bereits, dass dabei das soziale Wertesystem einer SEO nicht beeinträchtigt werden darf (IP10 $0^{775}$; IP $\beta^{776}$ ). Hierbei kann die eigene Grundhaltung zum Geld-Wert in den Regularien einer SEO verankert sein und dies kann Auswirkungen haben, inwieweit hierbei Druck oder Entlastung empfunden wird.

Die SEO von IP14 bildet dabei eine Extremhaltung ab in Form einer Lebensgemeinschaft aus sozial Benachteiligten, wobei die SEO für den Lebensunterhalt aller Betroffenen, die sich in der SEO engagieren und sich an einen transparent 
kommunizierten Wertekodex halten, aufkommt (IP14 ${ }^{777}$ ), einschließlich des Rentenalters (IP14 ${ }^{778}$ ). Hierbei verkörpert das Erwirtschaften von Geld einen zentral bedeutsamen Indikator der SEO-Zielsetzung, sich von Abhängigkeiten lösen zu können bzw. dauerhaft Unabhängigkeit zu erlangen, was letztendlich entlastend wirken kann.

Andere IP berichten, dass das Geldverdienen ein Antreiber sein kann, ein Vorbild-Exempel in der Öffentlichkeit zu statuieren $\left(\mathrm{IPG}^{779}\right.$, i. V. m. IPG ${ }^{780}$; IP10 ${ }^{781}$ ). Insofern ist im Einzelfall auf den Prüfstand zu stellen, inwieweit ,sozialen Zielen eine höhere Priorität bei[gemessen wird] als finanziellen“ (Bertelsmann Stiftung, 2013, S. 12) oder ob im Einzelfall eine Priorisierung der Zielsetzungen nicht mehr eindeutig feststellbar ist.

Darüber hinaus wird der Wunsch benannt, mit der eigenen Sozialunternehmung eine finanzielle Unabhängigkeit der eigenen Person zu erzielen (IP23 ${ }^{782}$, i. V. m. IP $23^{783}$ ). Jedoch kann dies ein Konfliktpotenzial innerhalb des sozialen Umfelds darstellen, insbesondere, wenn im eigenen Umfeld als Norm ausgerufen wird, dass das Generieren von Einnahmen mit gemeinnütziger Arbeit nicht vereinbar sei. Wird in der Öffentlichkeit - z. B. durch die Politik, SEO-U und mit wissenschaftlichen Fachbeiträgen - dieses restriktive Bild als allgemeingültig gezeichnet und gestreut und auf diese Weise gesellschaftlicher Konsens oktroyiert, dann kann dies den SE unter Rechtfertigungsdruck setzen oder aber provozieren, dass er in der Öffentlichkeit erst gar nicht zu seiner Absicht des Geldverdienens steht.

Wird in der Öffentlichkeit behauptet, dass gemeinnützige Organisationen stets auf der Basis ehrenamtlichen Engagements zu funktionieren haben, spiegelt dies eine einseitige Sichtweise wider und fokussiert nur einen bestimmten Bereich gemeinnütziger Organisationen wie z. B. Vereinsstrukturen, deren Mitglieder sich in ihrer Freizeit rein ehrenamtlich engagieren und die somit von SEO abzugrenzen sind $\left(\operatorname{IP}^{784}\right)$. So wenig, wie ehrenamtliches Engagement exklusiv und automatisch in Organisationsstrukturen mit anerkanntem Gemeinnützigkeitsstatus ausgelebt werden darf, so wenig ist ebenfalls der Rückschluss zulässig, dass jede gemeinnützige Organisation aus purem Ehrenamt zu bestehen hat. Mit dem letztgenannten Denkautomatismus entstehen ggf. Vorurteile zulasten von $\mathrm{SE}(\mathrm{O})$.

Wie bereits dargestellt, ist für mehrere IP von normativer Bedeutung, dass SE ihre soziale Wirksamkeit aufzuzeigen haben und in Abgrenzung zu KE dementsprechend zu belegen haben. Dabei reflektieren Befragte das SE-spezifische moralische Dilemma, ab wann sie welche Geldbeträge aus der SEO für ihr privates Wohlergehen verwenden dürfen. Demnach ergibt sich die Frage, inwieweit ein Gewinn zur Entwicklung weiterer sozialunternehmerischer Initiativen zum 
Abbau sozialer Benachteiligungsphänomene genutzt werden kann bzw. sollte entweder zur sich weiter spezialisierenden Neugründung oder auch zur breiteren Aufstellung im Sinne einer Diversifizierung (IP15 ${ }^{785}$ ). Denn eine Reinvestition kann gesellschaftlichen Mehrwert generieren, für die der SE mit seiner SEO angetreten ist (IP2 ${ }^{786}$ ). Beispielsweise musste sich im Anspruchsdenken von IP35 die Investition zuvorderst als gesellschaftlicher Mehrwert ausgezahlt haben. Bis dahin wirkte die selbst auferlegte Regel, sich privat zu verschulden (IP35 ${ }^{787}$ ).

Würde hingegen ein Selbstverständnis kommuniziert werden, dass es ebenfalls SE geben kann und geben darf, die infolge einer qualitativ hochwertigen, erreichbaren sozialen Wirkung durch ihr finanzielles Einkommen in finanzieller Sorglosigkeit leben, dann könnte dies eine psychologisch weniger belastende Ansage darstellen. Bei diesem Selbstverständnis würden sich SE also nicht auf Kosten anderer bereichern, sondern im Sinne einer Win-win-Situation sowohl anderen als auch sich selbst Gutes tun.

Eine eindeutige, allgemeingültige Beantwortung zur Adäquanz der SEbezogenen Mittelverwendung ist hierbei nicht möglich, so dass ein SE dies mit sich selbst ausmachen muss (IP3 ${ }^{788}$; IP2 $1^{789}$ ). Unbeantwortet bleibt, wie hoch der Betrag sein darf, den sich ein SE unter welchen Bedingungen für eigene Zwecke entnehmen darf: Darf es so viel sein, wie es in der Branche bei KE dem offiziellen Durchschnitt entspricht? Umsatzorientiert, dem Mindestlohn entsprechend oder z. B. zur Vermeidung des eigenen sozialen Abstiegs ( $\left(\mathrm{IP}^{790}\right)$ ? In Abhängigkeit von Projektvorhaben oder realisierten Maßnahmen zum Abbau sozialer Benachteiligung ( $\mathrm{IPc}^{791}$; IPJ ${ }^{792}$ )? Oder in Abhängigkeit von einem Mindestzeitraum des Bestehens der SEO bzw. aus weiteren, anderen Gründen?

Insgesamt zeichnet sich bei Befragten beim Thema des Geldverdienen-Dürfens ein problembehaftetes, zuweilen nicht widerspruchsfreies Bild ab (IP1 $\left.{ }^{793} ; \mathrm{IP}^{794}\right)$. Selbst wenn das Geldverdienen keinen Motivationsfaktor für einen SE darstellt (IP2 ${ }^{795}$ ), kann ein Konfliktfeld bestehen, wenn Geldverdienen als Wegnehmen des Geldes von anderen bewertet wird. Vergütungen können als nicht klug bis hin zu unmoralisch bewertet werden, da diese Mittel nicht mehr für die eigentliche Mission zur Verfügung stehen können (Kanter \& Sherman, 2016). Dabei ist zu differenzieren zwischen ,sich bereichern“ und „die eigene Lebenssituation sichern“ (IP2 $\left.{ }^{796}\right)$. Dennoch fällt, wie aufgezeigt, die konkrete Bewertung und Entscheidung schwer. Als weitere belastende Fragestellung wird dabei geschildert, ab wann ehrenamtliche Mitarbeiter aus Sicht des SE ausgebeutet werden (IP $\varepsilon^{797}$ ). Darüber hinaus verzichten hauptamtliche Mitarbeiter auf das, was sie von ihrer Leistung her verdienen, was ebenfalls SE belastet (IPÖ ${ }^{798}$ ).

Eventuell bedarf es einer Legitimation wie z. B. die eigene Familienversorgung oder auch ein Vorbild durch SE-Kollegen, den privaten Lebensunterhalt 
aus Einnahmen zu finanzieren (IP $\left.\alpha^{799}\right)$. Zudem kann für den SE den Konflikt untermauern, dass es ein relativ abstraktes Ziel darstellen kann, einen sozialen Missstand in der Welt abzubauen.

In der Gesamtbetrachtung ist somit infrage zu stellen, wenn behauptet wird, ,there is a wide consensus that SE combines an aspiration to make profit and the fulfillment of a social mission" (Kruse, Wach \& Wegge, 2018, S. 3, m. V. a. Bacq \& Jansen, 2011; Mair \& Martí, 2006).

\subsection{Persönliche finanzielle Absicherung}

Es gibt SE, die ihre finanzielle Situation frühzeitig reflektieren und vorausschauend denken. Beispielsweise werden messbare Zielsetzungen zur finanziellen Absicherung benannt (IP1 ${ }^{800}$; IP $25^{801}$ ). In anderer Weise wird geschildert, dass nicht nach Zielsetzungen gehandelt wird oder es findet keine messbare Zielsetzung statt, so dass Finanzierungsherausforderungen zum permanenten Begleiter $\left(\mathrm{IP} \varepsilon^{802}\right)$ oder verdrängt werden können (IP $\left.\gamma^{803} ; \mathrm{IP}^{804}\right)$. So werden SE medienwirksam als Positivbeispiel für erfolgreiches Sozialunternehmertum positioniert, leben aber am Existenzminimum $\left(\mathrm{IP}^{805}\right.$; IPU $\left.{ }^{806}\right)$. Gerade SE, die auf Fördergelder angewiesen sind, erleben Limitationen in der Vergütung, bedingt durch Zuwendungsregularien ( $\mathrm{IPg}^{807}$ ).

Demzufolge beuten sich SE selbst aus, stecken durchaus seit Jahrzehnten materielle Ressourcen in die SEO hinein (IP $\psi^{808}$, i. V. m. IP $\psi^{809}$ ) und verzichten dabei z. B. auf eine angemessene Altersvorsorge (IPU ${ }^{810}$ ). Diverse IP geben zu verstehen, dass sie im Rentenalter von Altersarmut betroffen sein werden (IPU ${ }^{811}$; IP $\gamma^{812}$; IP35 $5^{813}$; IP21 ${ }^{814}$; IP32 ${ }^{815}$; IP14 ${ }^{816}$ ), wobei sich die Befragten vereinzelt widersprüchlich äußern. Beispielsweise beklagt ein Befragter, dass nicht alle SE so konsequent unternehmerisch denken und handeln wie er. Aber der Widerspruch besteht darin, dass das Fundraisen nur auf die SEO bezogen wird, er sich selbst im Geldverdienen exkludiert (IPE ${ }^{817}$, i. V. m. IPE ${ }^{818}$; IPI ${ }^{819}$ ), was kein unternehmerisches Bild abgibt. Selbst bei einer schwarzen Null hat dieser Befragte das eigene Gehalt noch nicht mitberücksichtigt (IPI ${ }^{820}$ ). Sein unternehmerisches Denken und Handeln endet vor der eigenen Absicherung, so dass er sich diesbezüglich im Verhältnis zu seinen Mitarbeitern in eine inferiore Position versetzt.

Für das Sozialsystem zeichnet sich eine zu schulternde Belastungssituation ab, wenn der Staat ab dem Renteneintrittsalter für den SE sorgen muss. Derartige Belastungsdynamiken bleiben jedoch in sozialunternehmerischen Wirkanalysen unberücksichtigt. 
Wenngleich es vorkommt, dass IP ihre Eigenverantwortlichkeit erkennen und sich selbstwirksam erleben (IP18 ${ }^{821}$; IP25 $5^{822}$ ), wird deutlich, dass äußere Rahmenbedingungen zur Begründung von Selbstausbeutung herangezogen werden $\left(\mathrm{IP}^{823}\right)$. Obwohl Selbstausbeutung bei jeder Form des Unternehmertums auftauchen kann ( $\left.\operatorname{IP}^{824}\right)$, konnten mit den Ausführungen die Besonderheiten für SE verdeutlicht werden.

Im Rahmen der Datenauswertung geben von den Befragten lediglich zwei SE, die beide als Social Intrapreneure und somit im abgesicherten Angestelltenverhältnis tätig sind, zu verstehen, berufsbedingt keiner finanziell angespannten Situation im Privaten ausgesetzt zu sein $\left(\mathrm{IP} \eta^{825}\right.$, i. V. m. IP $\left.\eta^{826} \& \operatorname{IP}^{827} ; \operatorname{IP7}^{828}\right)$.

\subsection{Begriffliche Determinanten zur SE(0)-Zugehörigkeit}

Die Datenanalyse zeigt, dass ebenfalls diejenigen IP zur Beantwortung der zentralen Leitfrage beitragen, die zwar sozialunternehmerische Kriterien erfüllen, sich von der begrifflichen Zuordnung her allerdings nicht als SE verstehen (IP35 ${ }^{829}$; $\mathrm{IP}^{830}$; IPp ${ }^{831}$, i.V. m. IPp ${ }^{832}$ ), also den sozialunternehmerischen Begriff entweder nicht für sich nutzen (IP34 ${ }^{833}$; IPp ${ }^{834}$ ) bzw. in bestehenden Definitionen keinen Mehrwert für ihr eigenes Wirken erkennen (IPE ${ }^{835}$ ) oder den Begriff mit eigenen inhaltlichen Vorstellungen prägen (IPN ${ }^{836}$; IP10 ${ }^{837}$ ). Dabei kann der Unternehmensbegriff kulturell geprägt und konnotiert sein (IP ${ }^{838}$ ) und Erfahrungswerte können das Begriffsverständnis weiterentwickeln und verändern $\left(\mathrm{IP7}^{839}\right.$; $\left.\mathrm{IPB}^{840}\right)$. Zudem wird betont, dass in Netzwerken inkonsistente Begriffsvariationen zur Diskussion stehen, die keine klare, Sicherheit vermittelnde Richtung vorgeben können (IP $\left.\varepsilon^{841}\right)$.

Für SE, denen der sozialunternehmerische Begriff fremd ist bzw. die diesen nicht auf sich und ihre SEO beziehen, entsteht der Nachteil, dass ihnen demzufolge SEO-relevante Unterstützungssysteme gar nicht bekannt sein können (IP35 $\left.{ }^{842}\right)$.

Befragte SE erleben, dass ihre SEO sich konventionellen Strukturen bereits begrifflich nicht zuordnen lässt, sondern eine Zwitterposition einnimmt (IPR ${ }^{843}$ ). Zudem werden zwei „Parallelwelten“ beschrieben, wobei die KE-Welt und die SE-Welt bereits formal und in ihren strukturellen Kontexten Unterschiede aufweisen, die eine Vergleichbarkeit nicht erlauben (IP25 ${ }^{844}$ ). IP33 beschreibt für seine SEO hierbei eine hybride Struktur, die sich zusammensetzt aus ,urkapitalistisch“ und „Geld verschenken“ und dabei zugleich „von BEIDEM (.) das Original“ darstellt (IP33 ${ }^{845}$ ), wobei diese SEO das Ziel verfolgt, auf dem Weg des Geldverdienens soziale Teilhabebedingungen zu verbessern und dabei auch 
ökologisch bewusst zu handeln, so dass das Verschenken von Geld nicht in freier Auswahl, sondern unter der Bedingung der Förderung nachhaltiger Entwicklung passiert.

\subsection{Anspruchsniveau Problemlösung}

Das Anspruchsniveau einer Person wird bestimmt durch ihr angestrebtes Leistungsniveau; optimalerweise kann die betreffende Person ihr angestrebtes Leistungsniveau in das Verhältnis setzen zum bereits erlebten Leistungsniveau vergleichbarer Aufgaben (vgl. Atkinson, 1966). Dies kann bei neuartigen sozialunternehmerischen Vorhaben bestenfalls im Hinblick auf die Arbeitsbereitschaft und den Arbeitseinsatz möglich sein, jedoch nicht bezogen auf unbekannte, erst zu entwickelnde Arbeitsprozesse. Wenn darüber hinaus die Zielsetzung darin besteht, ein soziales Problem nicht nur zu bearbeiten bzw. die Absicht zu verfolgen, Lösungsansätze $\mathrm{zu}$ entwickeln und $\mathrm{zu}$ verbreiten (IPY ${ }^{846}$; siehe $\mathrm{z}$. B. Kimmitt \& Muñoz, 2018), sondern das soziale Problem tatsächlich zu lösen, also es zu eliminieren $\left(\mathrm{IPh}^{847}\right.$; IP $\left.\beta^{848}\right)$, dann kann dies den SE unter extrem hohen Leistungsdruck setzen und dabei dessen Leistungsfähigkeit mindern. Dementsprechend besteht bei der Unmöglichkeit, ein soziales Problem zu eliminieren, eine Leistungszieldiskrepanz (vgl. Atkinson, 1966), wobei ein implizit wie explizit verankerter Problemlösungsanspruch dazu führen kann, dass sich ein SE widersinnigerweise nur dann als hinreichend wirksam und leistungsfähig begreifen würde, wenn eben diese Illusion einträte.

Diese Problematik wird von SEO-U beispielsweise wie folgt zum Ausdruck gebracht: „Diese Publikation soll den verschiedensten kooperativen und lösungsorientierten Akteur*innen als Grundlage dienen, die sich der Lösung gesellschaftlicher Herausforderungen verschrieben haben“ (Osbelt, 2019); „Primäres Ziel von Social Entrepreneurship ist die Lösung gesellschaftlicher Herausforderungen, welches auch soziale und ökologische Aspekte beinhaltet" (Scharpe \& Wunsch, 2020, S. 11; in der Kernaussage wortgleich bei Hoffmann, Scharpe \& Wunsch, 2021, S. 13) und ,Gesellschaftliche Herausforderungen zu lösen ist der wichtigste Treiber für die DSEM-Social-Entrepreneurs" (Scharpe \& Wunsch, 2020, S. 7; vergleichbar z. B. bei Bertelsmann Stiftung, 2013, S. 19; vergleichbar ebenso mit IP18 ${ }^{849}$; $\mathrm{IPb}^{850}$; zur unrealistischen Betrachtungsweise siehe $\mathrm{z}$. B. IP2 $1^{851}$ ).

Im Rahmen des Gründungsmonitors der KfW wird dies bei der Datenerhebung differenzierter betrachtet, da die von den Befragten zu beantwortende Fragestellung hierzu lautet: ,Zweck meiner selbstständigen Tätigkeit ist es, einen 
spezifischen Beitrag zum Umweltschutz oder zur Lösung eines gesellschaftlichen Problems zu leisten“ (Metzger, 2019, S. 2).

Bei der Annahme, ein soziales Problem könnte mithilfe der SEO beseitigt und der Markt demzufolge uneingeschränkt gesättigt werden, arbeiten SE aktiv an ihrer eigenen Arbeitslosigkeit (IP21 ${ }^{852}$; IPH ${ }^{853}$; IP20 $0^{854} ; \mathrm{IPq}^{855}$ ). Dementsprechend gipfelt organisationales Wachstum bei der Zielsetzung, soziale Benachteiligung abzuschaffen ( $\operatorname{IPm}^{856}$ ), zwangsläufig in der Konsequenz des Unternehmenstods.

Doch erweist sich das Ziel, ein Problem zu lösen, als nicht nachvollziehbar, wenn sich soziale Benachteiligungen, denen sich SE widmen, nicht lösen, sondern nur reduzieren oder verändern lassen (IP21 ${ }^{857}$; IPm ${ }^{858}$; IP25 ${ }^{859}$; IP19 ${ }^{860}$; siehe auch Abschn. 7.3 die Diskussion zum Haltungsziel). Nicht die Lösung eines gesellschaftlichen Problems ist das Ziel dieser IP, sondern einen Lösungsweg für den sozial wirksamen Umgang mit dem entsprechenden gesellschaftlichen Problem zu finden und zu gehen. Diese grundsätzliche Überlegung wird von Befragten durchaus verdrängt $\left(\mathrm{IPz}^{861}\right.$; leicht relativiert bei IP $\left.\psi^{862}\right)$. Anhang $\mathrm{K}$ im elektronischen Zusatzmaterial zeigt als Ergebnis einer qualitativen Inhaltsanalyse tabellarisch zusammengefasst für alle Befragten, was an dieser Stelle diskutiert wird: Kein IP, der das Ziel der Problemlösung verfolgt, hat dieses Ziel erreicht. Im Rahmen einer zusätzlichen, studienbegleitenden Recherche konnte ebenfalls kein SE identifiziert werden, der mithilfe seiner SEO ein soziales Problem eliminieren konnte.

Eine Problemlösung im Sinne einer Vision ohne Erreichbarkeitsanspruch kann hingegen sinnvoll erscheinen. Denn dies würde ein weiteres Dilemma auflösen: dass die als erreichbar gemeinte Zielsetzung, sich abzuschaffen, nicht nur den Höhepunkt einer SEO-Entwicklung darstellen würde, sondern per se den Abschluss aller unternehmerischen Aktivitäten, was eine SEO bereits in der Planung einen Projektstatus verleiht, so dass klassische Projektphasen anzusetzen sind (IP25 ${ }^{863}$ ). Unternehmen hingegen wollen sich als System aufrechterhalten, was die organisationsimmanente Anpassungsbereitschaft des Systems an neue Umweltanforderungen einfordert (systemtheoretisch begründet z. B. bei Luhmann, 2011, S. 12 ff.; Luhmann, 1984). Wenngleich theoretisch gegenargumentiert werden könnte, dass sich unmittelbar nach Beendigung eines Projekts ein neues anschließen kann, besteht dennoch eine begriffliche Abgrenzung, die sich dem Unternehmerischen unterzuordnen hat. Insofern kann dies im Mindset lediglich eine Kompensation darstellen und zu Beeinträchtigungen im unternehmerischen Selbstverständnis führen (weiter ausgeführt in Abschn. 8.11). Aus unternehmerischer Sicht scheint angebracht, von vornherein im Produktlebenszyklus zu denken, der während der Produktentwicklung in aller Regel hohe 
Investitionskosten einfordert. Erweist sich sodann ein sozialunternehmerisches Konzept als praxistauglich und sinken die Stückkosten nach der Produkteinführung bei steigendem Absatz, dann kann sich daraus eventuell eine Cash Cow entwickeln. Für den Fall, dass ein SE dieses Niveau erreicht hat und sich die Marktsättigung prognostizieren lässt, sollte der SE Vorsorge getroffen haben, indem er im sozialunternehmerischen Verbesserungsprozess bereits Neuoder Weiterentwicklungen vorangetrieben hat, die seiner sozialen Zielsetzung entsprechen.

Eventuell ließe sich zudem infolge des Verzichts auf einen unerreichbaren Problemlösungsanspruch das bereits thematisierte Dilemma zur Positionierung gegenüber dem Geldverdienen relativieren: das Bestreiten des eigenen Lebensunterhalts aus dem sozialunternehmerischen Engagement einzukalkulieren und dies von vornherein nach selbst festgelegten Kriterien messbar zu gestalten.

Wie bereits diskutiert, zeigen alle Befragten im Hinblick auf ihr sozialunternehmerisches Engagement auf, erfolgsmotiviert zu sein, wobei sie sich in unterschiedlicher Ausprägung wünschen, dass ihre Leistung anerkannt wird (vgl. Abschn. 8.1 bis 8.4; vergleichbar mit Boluk \& Mottiar, 2014). Atkinson (1966) postulierte grundsätzliche Annahmen zur Leistungsmotivation. Demnach wollen Personen einen größtmöglichen Nutzen aus ihrem Handeln ziehen, demgemäß mit ihren Handlungen Freude und Stolz maximieren und leidvolle Erfahrungen vermeiden bzw. minimieren, was keinesfalls eine widerspruchsfreie Gesamtsituation darstellen muss (IP $\left.\alpha^{864}\right)$. Damit wird auf Basis einer Vernunftentscheidung erforderlich, für das Ausrichten des eigenen Verhaltens den subjektiven Wert eines Ziels ebenso zu kalkulieren wie die Erwartungswahrscheinlichkeit dieses Ziels. Auf dieser Basis leitet Atkinson ein Modell zur Risikowahl ab:

$$
\begin{array}{ll} 
& {[\text { Tendenz, Erfolg anzustreben }]} \\
+\quad & {[\text { Tendenz, Misserfolg zu vermeiden }]} \\
\hline=\quad & {[\text { resultierende Verhaltenstendenz }]}
\end{array}
$$

In der Interpretation identifiziert Atkinson zwei Gruppen: (1) Erfolgsmotivierte, die bevorzugt Aufgaben mit mittlerer Schwierigkeit wählen, da sie auf diese Weise die beste Balance aus Erwartung und Wert erfahren, und (2) Misserfolgsvermeidungsmotivierte, die entweder besonders leichte oder besonders schwierige Aufgaben wählen. 
Nach Atkinson werden erfolgsorientierte SE eine schwierige Aufgabe nach erlebtem Misserfolg als noch schwieriger empfinden, so dass sie zur Vermeidung von Misserfolgserlebnissen an der Problemlösung nicht festhalten sollten, sondern zur Aufrechterhaltung ihrer Arbeitsmotivation zu bewältigende Aufgaben finden und wählen sollten. Bei erfolgsmotivierten SE kann das verzerrte Anspruchsniveau, ein soziales Problem zu eliminieren, zu einem inneren Ausbrennen führen bzw. dies begünstigen (vgl. z. B. Kearney, Dunne \& Wales, 2020; Tuominen-Soini, 2012; Parker \& Martin, 2011).

Eine ähnliche Problematik zeigt sich in Bezug auf Skalierung und ebenso beim Anspruchsdenken in Bezug auf die Innovationskraft. Beide Aspekte werden nachfolgend vertieft.

\subsection{Anspruchsniveau Skalierung}

In Erinnerung gerufen wird die Ausführung in Abschnitt 5.10 zur SEO-U-Arbeit im Hinblick auf Skalierungsansprüche: Dargestellt wurde u. a., dass entweder die Steigerung der sozialen Wirkung im Mittelpunkt von Hochskalierung stehen kann $\left(\mathrm{IPR}^{865}\right.$; IP2 $27^{866}$, i. V. m. IP2 $7^{867} \&$ IP2 $7^{868}$; IP16 ${ }^{869}$ ) oder dass mit Hochskalierung ein Größenwachstum der SEO verbunden werden kann und eventuell darauf aktiv hingearbeitet wird (IP26, ${ }^{870}$ i. V. m. IP26 $6^{871}$ ). Denkbar erscheint dabei, dass Problemlösungsdruck einen Druck zum Hochskalieren nach sich ziehen kann.

Erinnert wird insbesondere an die Ausführung zur SEO von IP13, deren Hauptsponsor sich nicht an eine mündliche Vereinbarung gehalten hat (IP13 ${ }^{872}$ ), was zu massiven Mittelkürzungen führte (IP13 ${ }^{873}$ ), die wiederum schwerwiegende SEO-interne Veränderungen mit sich brachten und zu enormen Anstrengungen bei der Mittelakquise führten. IP13 gibt in ihrer Ausführung zu verstehen, dass bei den SEO-Mitarbeitern diese Veränderung starke Irritationen hervorgerufen hat, zumal arbeitsplatzbezogene Verpflichtungen top-down beschlossen und kommuniziert wurden, was auf Mitarbeiterseite als Bruch zur bis dahin gelebten, auf Mitsprache bedachten Unternehmenskultur empfunden wurde (IP13 ${ }^{874}$ ) und in der Folge als unfair und als nicht respektvoll ihnen gegenüber aufgefasst wurde. Auf sozioemotionaler Ebene führte dies zur Verletzung des psychologischen Vertrags zwischen Arbeitgeber und Arbeitnehmer (vgl. Morrison \& Robinson, 1997, weiter ausgeführt in Abschn. 9.4). In der Folge kam es zu Einbrüchen in der Arbeitsleistung, die Einbußen im Organizational Citizenship Behavior widerspiegeln (Organ, 1988): IP13 berichtet von nachlassendem Mitarbeiterengagement, insbesondere bezogen auf das selbstbestimmte Engagement der Mitarbeiter über deren 
Pflichtaufgaben hinaus, also hinsichtlich Aufgaben, die außerhalb des formalen Anreizsystems liegen.

Diese akute Neuausrichtung der SEO auf marktwirtschaftliche Performance stellt eine Mission Drift dar: Mitarbeiter können sich nicht mehr wie gehabt mit ihrer SEO identifizieren (Porter \& Kramer, 2011, S. 76; Battilana \& Lee, 2014, S. 414 f.; Chell, Spence, Perrini \& Harris, 2016; Marshall, 2011). Wenngleich die SEO weiterhin ihre sozialunternehmerische Mission besitzt, kommt es zu Verschiebungen und Neuausrichtungen bei der Zielsetzung. Dabei ist im sozialunternehmerischen Alltagsgeschehen für die Mitarbeiter die sozialunternehmerische Mission nicht mehr eindeutig erkennbar. Hierauf zeigen Mitarbeiter Reaktanz (zu Beziehungs- und Prozessdynamiken bei Mission Drift vgl. z. B. Grimes, Williams \& Zhao, 2019).

Befragte zeigen auf, dass organisationales Größenwachstum struktureller Anpassungsprozesse der SEO bedarf und vor allem finanzieller Mittel, so dass der Finanzierungsdruck durch organisationale Hochskalierung dauerhaft steigt (IP29 ${ }^{875}$; IP32 $2^{876}$; IP21 ${ }^{877}$ ). Dabei kann ein Hochskalieren der SEO die Leistungsqualität beeinträchtigen und z. B. zur höheren Fehlerrate führen oder einhergehend mit stärkerem Leistungsdruck zur höheren Bereitschaft für regelwidriges Verhalten (vgl. Tintor, 2012; Baeckmann, 1998, S. 40 ff.; Fürst \& Wieland, 2004).

Ebenso zeigen die Ausführungen von Befragten, dass aus Größenwachstum ein qualitatives Hochskalieren hervorgehen kann bzw. dies damit verbunden wird (IP27 $7^{878} ; \mathrm{IP}^{879} ; \mathrm{IP} \gamma^{880} ; \mathrm{IP} \tau^{881}$; IP31 ${ }^{882}$ ). Wenngleich organisationales Wachstum die SEO finanziell gefährden kann, können eventuell aus einer zusätzlichen inhaltlichen Ausrichtung zur Verbreitung der sozialen Wirkung Einnahmen generiert werden, die für das Absichern von organisationalem Wachstum existenzsichernd eingesetzt werden können. Eine derartige Win-win-Situation beschreibt IP27 (IP27 $7^{883}$ ).

Somit beeinflussen die Prozesse des Hochskalierens die finanzielle Gesamtsituation der SEO (IP35 ${ }^{884}$ ), wobei die finanzielle Situation im Zusammenhang der sozialen Wirkung zu bewerten ist (IP $\theta^{885}$; IP35 ${ }^{886}$ ). Um das Hochskalieren der Qualität zu sichern, bedarf es beim Kooperationsmanagement klarer Prozessbeschreibungen (IP ${ }^{887}$; IP35 ${ }^{888}$ ), die auf Instrumenten zur transparenten Messung der sozialen Wirkung beruhen sollten. In der Gesamtbetrachtung stellt sich die grundsätzliche Frage, ob die SEO als Organisationsstruktur skalieren soll oder deren soziale Wirksamkeit bzw. beides (IPY ${ }^{889}$; IPH $^{890}$ ).

Vergleichbar zum zentral verankerten Anspruch der Steigerung sozialer Wirkung will IP $\tau$ ebenso ihre SEO vor allem in ihrer Qualität der Dienstleistung hochskalieren, nicht aber in ihrer Quantität. Aufgrund von Negativberichten 
zu Beratungsangeboten für $\mathrm{SE}(\mathrm{O})$, die $\mathrm{IP} \tau$ von $\mathrm{SE}$ zugetragen wurden und die sich mit kritischen Ausführungen in Abschnitt 5.12 decken, schließt IP $\tau$ für ihre SEO kategorisch eine Zusammenarbeit mit wirtschaftlich ausgerichteten Unternehmensberatern aus (IP $\left.\tau^{891}\right)$. Im Umkehrschluss könnte ein breiteres Grundverständnis von Wachstum, das auf Qualitätsmerkmalen beruht und eine umfassende Finanzanalyse zulässt und dabei auch das Skalierungsverständnis breit fasst, dazu führen, dass IP $\tau$ einen besseren Zugang zu ihrer wirtschaftlichen Ausrichtung bekommt. Es erscheint erstrebenswert, dass SE nicht unter dem Druck stehen, Größenwachstum generieren zu müssen, aber bereit sind und Unterstützung darin erfahren, sich konstruktiv mit der Finanzplanung und -gestaltung ihrer SEO auseinanderzusetzen.

Erinnert wird abermals an die Ausführung in Abschnitt $5.10 \mathrm{zu}$ dem Wohnund Betreuungskonzept für eine Kleingruppe sozial benachteiligter Menschen $\left(\mathrm{IPJ}^{892}\right.$, i. V. m. IPJ ${ }^{893}$ ), das neben der dauerhaften direkten wie indirekten sozialen Wirkung ebenso kontinuierlich Einsparungen von Steuergeldern ermöglicht, über Jahre gesehen im Millionenbetrag. Dabei kann der Eindruck entstehen, dass der betreffende SE mit seinem konsequenten Blick auf Einzelfälle die Möglichkeit verkennt, ein Konzept zu entwickeln, das auf vergleichbare gesellschaftliche Kontexte übertragbar ist (IPJ ${ }^{894}$; vergleichbar bei FG5Z1576-1582 ${ }^{895}$ ). Jedoch kann die Konzentration auf den Einzelfall erst den Tiefgang der sozialen und in der Folge die wirtschaftliche Wirkung ermöglichen. Hierbei passen die folgenden Leitfragen der Bertelsmann Stiftung (2013) zur Skalierung sozialer Wirkung: „Ist die Skalierung der sozialen Wirkung Ihres Sozialunternehmens stark mit Ihrer Person verknüpft? Haben Sie beispielsweise spezifische Kompetenzen, die nur Sie und keine Ihrer Mitarbeiter beherrschen? Falls ja, würde eine Ausdehnung in andere Regionen für Sie wertvolle Zeit und Reisekosten zu anderen Standorten bedeuten, die Sie sinnvoller in eine regionale Fokussierung Ihrer Aktivitäten investieren sollten“ (S. 34).

Soziale Wirksamkeit ist demzufolge nicht aus der Unternehmensgröße ableitbar, sondern aus der Qualität und Stabilität der Wirkkette. Im zuvor genannten Beispiel zum Wohn- und Betreuungskonzept wird deutlich, dass die Wirkkette eine finanzielle und eine soziale Ebene hat. Sozialunternehmen wirken also auf zwei verschiedenen Ebenen, die zwar Berührungspunkte aufweisen, aber keine direkt zusammenhängende Einheit bilden. Im hier diskutierten Beispiel ist der Schwellenwert der sozialen Wirksamkeit ab dem ersten Kunden bereits erreicht. Die finanziell-unternehmerische Wirksamkeit kann zeitversetzt eintreten, wenn sich im Rahmen der Gründung Entwicklungskosten wie z. B. für den Umbau barrierefreier Wohnungen ergeben, die aus Steuergeldern finanziert worden sind und sich erst amortisieren müssen. 
Eventuell kann das Vermeiden, in den Organisationsstrukturen zu wachsen, mit dem Vorteil verbunden sein, dass kleine SEO qualitativ andere Möglichkeiten haben, etwas auszuprobieren. Zudem geben Befragte zu verstehen, dass bei großen Strukturen andere Erwartungshaltungen entstehen können, die weniger Flexibilität ermöglichen (IP $\left.\gamma^{896}\right)$. Ebenso wird betont, dass Reduktion der Quantität - z. B. Internationalität aufzugeben - ein Intensivieren der Qualität mit sich bringen kann. Hierbei kann tiefgründiger als zuvor gearbeitet werden und diese Entwicklung kann zudem neue Arbeitsressourcen hervorbringen, die in anderer Form investiert werden können (IPÖ ${ }^{897}$ ).

Oldenburg hebt zur obigen Ausführung passend hervor: ,Social entrepreneurs denken Wachstum anders: Statt nur durch Organisationswachstum steigt ihre Wirkung vor allem indirekt durch Nachahmer, Kooperationen, Regelveränderungen. Sie maximieren die Wertschöpfung im Gesamtsystem statt in der eigenen Organisation" (2011, S. 120). Befragte geben zu verstehen, dass Nachahmer in der Tat gewünscht sein können und gefördert werden (IPc ${ }^{898}$; IP27 ${ }^{899}$ ). Dementsprechend können Nachahmung und Kooperationen den SE entlasten, da er weder Retter eines sozialen Missstands sein kann noch sein muss (vgl. Abschn. $7.2 \mathrm{zu}$ Marktabhängigkeiten). Allerdings ist Replizierbarkeit nicht immer möglich (Bertelsmann Stiftung, 2013, S. 35) und falls doch, kann dabei Wettbewerbsdruck und somit ein Spannungsfeld im Kooperationsmanagement entstehen (vertiefend diskutiert in Abschn. 9.2 im Kontext von Coopetition).

In der Gesamtbetrachtung stellt sich die Frage, inwieweit Abstriche in der Produkt- bzw. Dienstleistungsqualität und somit in der sozialen Wirksamkeit in Kauf genommen werden sollen bzw. können oder ob die Abstriche bei der unternehmerischen Absicherung zu erfolgen haben (IP10 $0^{900}$ ). Dieses Spannungsfeld stellt einen kontinuierlichen Reflexionsprozess dar.

IP14 verdeutlicht, dies im Fließgleichgewicht nach zuvorderst definierten Prioritäten gewissenhaft zu managen. Das sozialunternehmerische Konzept seiner SEO beinhaltet u. a., Obdachlose, die alkoholabhängig sind und die sich von ihrer Abhängigkeit befreien wollen, mit Wohnraum zu versorgen und in eine alkoholabstinent lebende Gemeinschaft aufzunehmen. Bei seinem Fließgleichgewichtsmanagement greifen innerhalb der SEO selbstauferlegte Regeln, z. B. Eigentum zu erwerben und grundsätzlich keine Gebäude anzumieten. Dies erweist sich in Zeiten besonders hohen Zuspruchs als unternehmerische Wachstumsbremse, nicht aber als soziale Wirkungsbremse, da die soziale Zielsetzung, alle um Unterstützung bittende Menschen aufzunehmen und ihnen zu ermöglichen, sich aus ihrer Alkoholabhängigkeit und aus ihrer sozialen Benachteiligung zu befreien, gewährleistet wird. Somit kann zwar der Lebenskomfort im Alltag in 
Zeiten besonders hohen Zuspruchs leiden, wenn für verhältnismäßig viele Menschen dementsprechend wenig Wohnraum zur Verfügung steht. Dennoch achtet IP14 konsequent darauf, dass die soziale Zielsetzungspflicht erfüllt wird; aber in der Kür, der B-Note im alltäglichen Zusammenleben, kalkuliert er zur unternehmerischen SEO-Sicherung Einschränkungen ein, solange darunter die soziale Zielsetzung nicht in Mitleidenschaft gezogen wird (IP14 ${ }^{901}$ ). Dies passiert auf Basis eines klaren Priorisierungsmanagements (IP14 ${ }^{902}$ ), wobei das Wirkungsverständnis stets darin besteht, Abhängigkeiten, die ein Gefährdungspotenzial für Personen und für die SEO beinhalten, zu reduzieren bzw. zu vermeiden. Als Winwin-Situation wirkt dabei, dass alle Personen, die von der SEO-Gemeinschaft aufgenommen worden sind, auf Basis ihrer persönlichen Kompetenzen und Möglichkeiten ihren aktiven Arbeitsbeitrag leisten, die der SEO direkt zugutekommt. Auf diese Weise können Wirkungsempfänger verlorengegangene Selbstwirksamkeitsüberzeugung zurückgewinnen und sich als „systemrelevant“ erleben. Die aus ihrer Arbeitsleistung hervorgegangenen Ergebnisse kommen der Stabilisierung der SEO direkt zugute. Da in der SEO sichergestellt wird, dass die soziale Zielsetzung für jeden Wirkungsempfänger erreichbar und realisierbar gestaltet wird, ist dementsprechend dringend und wichtig die Zunahme der Skalierung sozialer Wirkung priorisiert. Hierbei hat sich das Wachstum der Organisationsstrukturen unterzuordnen und ein Scaling deep vollzieht sich durchdacht innerhalb gegebener Ressourcenrestriktionen.

Befragte heben hervor, dass ein umfassendes Skalierungsverständnis inkludiert, eine SEO in wirtschaftlich schwierigen Zeiten am Markt am Leben zu erhalten, also ggf. in der Lage zu sein, Organisationsstrukturen zu reduzieren, ohne die SEO-Existenz zu gefährden (IP $\gamma^{903}$ ). Fixkosten wie Kredite, Miet- und Personalkosten müssen auch in wirtschaftlich schlechten Zeiten bedient werden. Runterskalieren stellt in aller Regel eine hohe finanzielle Herausforderung dar. Feste Strukturen, die der SE nicht auflösen oder abstoßen kann, sind sinnvoll zu nutzen. Dies kann mit sich bringen, dass eine hiervon betroffene SEO einem tiefgründigen sozialunternehmerischen Transformationsprozess ausgesetzt ist. Insofern ist zu prüfen, inwieweit beim Runterskalieren eine Chance für neue, andere und eventuell innovative Maßnahmen besteht, aus denen z. B. ein neues sozialunternehmerisches Standbein hervorgehen kann (IP16 $6^{904}$, i. V. m. IP16 ${ }^{905}$ ).

Ein Spagat vermag darin zu bestehen, dass die SEO sowohl den neuen Umfeldbedingungen gerecht wird als auch der sozialen Zielsetzung treu bleibt. Dementsprechend ist der Change-Prozess in seiner Entwicklung zu meistern. Dies setzt voraus, sämtliche sozialunternehmerischen Aktivitäten auf ihre Tauglichkeit und Wirkung im Hinblick auf einen kontinuierlichen Verbesserungsprozess zu 
evaluieren, zumal Wachstumsziele von vielen Einflüssen abhängig sein können, so dass sie nicht rigide, sondern flexibel gestaltet werden sollten (IPT $\left.{ }^{906}\right)$.

IP2 lotet sein Fließgleichgewichtsmanagement ebenfalls auf den zwei Waagschalen soziale Rendite versus finanzielle Rendite aus: IP2 geht konsequent individuell auf die Bedürfnisse seiner Zielgruppe ein (IP2 ${ }^{907}$ ). Sein Geschäftsmodell, das sich der Lebenssituation sozial entwurzelter Menschen mit schwersten körperlichen und psychischen Traumatisierungen widmet, verlangt ihm dies geradezu ab. IP2 erhält für jeden Wirkungsempfänger eine Einzelförderung. Diese werden von Ämtern beschieden. Sie erwarten bei Einzelbetreuungen keine Abweichung vom Standard; genau diese provoziert aber IP2. Er bricht somit eindeutige Konventionen und geht damit ein existenzbedrohendes Risiko ein (IP2 ${ }^{908}$; IP2 ${ }^{909}$; $\mathrm{IP} 2^{910}$ ). Demgemäß werden niedrigere Einnahmen in Kauf genommen, wenn sich dies positiv auf die Zielgruppe und auf die soziale Wertschöpfung auswirkt (vgl. Austin, Stevenson \& Wei-Skillern, 2006).

Unter finanziellem Aspekt hätte sich IP2 sein Unternehmerdasein deutlich leichter machen können und eine „Betreuung von der Stange“ ohne Arbeitsaufwand über Gebühr im Sinne der Finanzierungsgrundlage der Ämter leisten können. Allerdings sind für IP2 nicht die Geldgeber die Auftraggeber, sondern die Wirkungsempfänger der Dienstleistung. Dementsprechend richtet er einzelfallbezogen die Zielsetzung mit sozialer Wirkung aus. Dabei gibt ihm der Erfolg seiner SEO-Tätigkeit und der seiner Mitarbeiter Recht, da die Wirksamkeit sowohl im Hinblick auf soziale Rendite als auch auf eine gesellschaftsbezogene finanzielle Rendite belegt werden kann: Bislang wurden alle Klienten der Einrichtung mit einer Ausbildung in den ersten Arbeitsmarkt vermittelt und IP2 betonte im Anschluss an das Interview, dass Einrichtungen mit standardisiertem Betreuungsangebot dies nicht schaffen. Die SEO hat bewirkt, dass keine Hartz-IV-Empfänger aus dem Betreuungsprogramm hervorgehen, wie sonst eher üblich. Die ehemaligen Klienten zahlen heute Steuern. Gesellschaftlich hat sich diese Dienstleistung bereits vielfach ausbezahlt. Diese finanzielle Rendite wird jedoch nicht als Motivation von IP2 benannt. Erinnert wird an die Ausführungen in Kapitel 6 zur Arbeitsweise von Behörden und Entscheidungen der Politik; dabei wertschätzen staatliche Strukturen weder die finanzielle noch die soziale Rendite, so dass beide Erfolgskriterien in der Praxis kein Argument darstellen können, die Arbeit der SEO zu fördern.

Abschließend wird noch einmal an die Diskussion des Anspruchsniveaus zur Problemlösung erinnert: Thematisiert wurde, dass Hochskalierung bei der Zielsetzung, soziale Benachteiligung abzuschaffen ( $\operatorname{IPm}^{911}$ ), zwangsläufig in der Konsequenz des Unternehmenstods gipfelt. Wenngleich beschrieben wurde, dass im Rahmen dieser Untersuchung keine SEO identifiziert werden konnte, die ein 
soziales Problem wirklich gelöst hat, soll theoretisch in Betracht gezogen werden, dass eine SEO im Falle der sozialen Problemlösung ihren unternehmerischen Höhepunkt und Todesfall zugleich erlebt, es sei denn, die Problemlösung wird als extreme Form des Runterskalierens angesehen und geeignete Maßnahmen zur Sicherung der SEO-Existenz werden frühzeitig ergriffen, so wie oben dargestellt.

\subsection{Anspruchsniveau Innovationsentwicklung}

Wie in Kapitel 2 beschrieben, werden durch SEO-U und durch wissenschaftliche Publikationen Rahmenbedingungen für das Verständnis von Social Entrepreneurship gesetzt (vgl. z. B. Morris, Santos \& Kuratko, 2020). In Abschnitt 5.10 wurde bereits ausgeführt, dass diese Rahmenbedingungen - beispielsweise die Voraussetzung, eine Innovation hervorzubringen (IP $\xi^{912}$ ) - Segregationseffekte provozieren können. Demzufolge können SE daran gehindert werden, ein sozialunternehmerisches Selbstverständnis und Selbstbild zu entwickeln. Innovation wird nicht von allen SE mit Sozialunternehmertum zwangsläufig in Verbindung gebracht, sondern kann hiervon sogar abgegrenzt werden $\left(\mathrm{IPb}^{913}\right.$; IP2 $7^{914}$; IP2 $1^{915}$ ).

Aufgezeigt wurde in Kapitel 2 ebenfalls, dass der Innovationsbegriff mit unklarem bzw. uneinheitlichem Verständnis genutzt wird (im internationalen Vergleich siehe z. B. Adro \& Fernandes, 2020; Casini, Bensliman, Fossati, Degavre \& Mahieu, 2018). Dies trifft ebenso auf die Befragten zu (IP27 $7^{916} ; \mathrm{IPh}^{917} ; \mathrm{IPO}^{918}$; $\mathrm{IPA}^{919}$ ). Unter anderem wird nicht zwischen Invention und Innovation differenziert. Etwas zu erfinden, ohne dies im Markt einzuführen, stellt zunächst eine Invention dar; sie muss noch keine Innovation verkörpern, denn ,there is no necessary connection between the two functions. The inventor produces ideas, the entrepreneur ,gets things done“" (Schumpeter, 1947, S. 152).

Einerseits ist mit der Entwicklung eines Prototyps bis zur angenommenen Marktreife ohne Markteinführung noch nicht sichergestellt, dass ein Markt besteht bzw. die „Innovation“ vom Markt angenommen wird (IP1 ${ }^{920}$ ). Andererseits wirkt die Innovationskraft bereits ab der ersten Person, die hiervon profitiert, also unabhängig von einer Hochskalierung (IP21 ${ }^{921}$, i. V. m. IP21 ${ }^{922}$; IP20 $0^{923}$ ). Eventuell sind kulturelle bzw. geografische Anpassungen erforderlich (IPt ${ }^{924} ; \operatorname{IP}^{20} 0^{925}$; $\mathrm{IP}^{2} 7^{926} ; \mathrm{IPk}^{927}$; $\mathrm{IPY}^{928}$ ).

In der Gesamtbetrachtung besteht sogar eine Vielzahl an Definitionsansätzen zu sozialer Innovation, die zum Teil deutlich voneinander abweichen (siehe hierzu z. B. Howaldt \& Schwarz, 2015). Beispielsweise kann eine unmittelbare 
Bezug- und Einflussnahme auf Tradition und wertgebundene Überzeugungen vorausgesetzt werden (Drucker, 1957) oder Transformationsprozesse im Hinblick auf politische Macht durch soziale Bewegungen werden als Bedingung vorgegeben (Lapierre, 1977). Ein weiteres Grundverständnis bezieht sich auf die Analyse sozialer Konflikte und die Entwicklung von Lösungsstrategien (Neuloh, 1977).

Fueglistaller et al. definieren Innovation als ,erstmalige Anwendung einer neuen Problemlösung oder wiederholender Problemlösungstechnik, die darauf gerichtet ist, Unternehmensziele auf neuartige Weise zu erfüllen“ (2019, S. 102). Bei diesem Verständnis sind nicht neue Unternehmensziele innovativ, sondern neuartige Wege zum Erreichen der Unternehmensziele. Dabei kann unterschieden werden zwischen Produkt- und Dienstleistungsinnovation, Prozessinnovation, Geschäftsmodellinnovation und technischen Innovationen, wobei sich Letztere in Material- und Produktinnovationen unterscheiden lassen. Soziale Innovation wird allerdings zuweilen von technischen Innovationen abgegrenzt, da technische Innovationen den ökonomischen Nutzen in den Mittelpunkt stellen, was bei sozialer Innovation nicht der Fall ist. Brooks (1982) typologisiert hierbei zwischen technischer, soziotechnischer und sozialer Innovation. Zudem kann bei sozialer Innovation die Differenzierung zwischen technologischen, betriebswirtschaftlichen und sozialen Innovationstreibern hinken: Befragte betonen, dass es immer um die Wirkung für den Menschen gehen muss (IP21 ${ }^{929}$ ).

Eine im Hinblick auf den Problemlösungsanspruch gemäßigtere Definition besagt, dass soziale Innovationen als neuartige Lösungsansätze auf strukturellorganisationaler Ebene oder als Lebensstil aufgefasst werden, die machbare Prozesse zur Bewältigung sozialer Herausforderungen gewährleisten und dabei gesellschaftlichen Nutzen erwirken (Gillwald, 2000; Zapf, 1989, S. 177; vergleichbar mit $\mathrm{IPJ}^{930}$ ). Befragte zeigen dabei auf, dass der plötzlich eingetretene persönliche Bezug zum Thema den Zugang zu neuen Wegen ermöglichen kann, weil dadurch erst Perspektivwechsel eingenommen wurden $\left(\operatorname{IP} \lambda^{931}\right.$, i.V. m. $\left.\operatorname{IP} \lambda^{932}\right)$.

Gillwald (2000) zieht zum Begriffsverständnis für Innovation das Patentrecht heran und betont hierbei, dass sowohl eine unbewusste als auch eine bewusste Nachahmung von bereits existierenden Verfahrensweisen nicht mit Innovation vereinbar sein kann, da ,absolute Neuartigkeit zu den Eigenschaften [gehört], die vor einer Verleihung von Urheberrechten auf technische Erfindungen nachgewiesen sein müssen" (S. 10; vergleichbar mit $\operatorname{IPc}^{933}$ ), so dass die Übernahme bestehender Innovationen für die eigene Entwicklungsarbeit verweigert werden kann (zum „Not-invented-here-Syndrom“ siehe Katz \& Allen, 1982; Hauschildt, Salomo, Schultz \& Kock, 2016, S. 52 f.). 
Allerdings wird die Renaissance altbekannter, jedoch in Vergessenheit geratener Vorgehensweisen i. S. v. Wiederentdeckungen zuweilen als „Nacherfindungen" und als Innovation aufgefasst (Gillwald, 2000, S. 10, vergleichbar mit $\mathrm{IP} \mu^{934}$ ), zumal ,,[e]s kein vollständig Neues [gibt], losgelöst von jeder Beziehung zum Alten, Überlieferten“ (Bechmann \& Grunwald, 1998, S. 4 f.). Befragte geben zu verstehen, dass bei diesem Grundverständnis jede Form eines gesellschaftlichen Mehrwerts als Innovation bezeichnet werden kann $\left(\operatorname{IPt}^{935}\right)$ und Innovation kann insofern synonym zu Kreativität aufgefasst werden, was zugleich ein transdisziplinäres Grundverständnis aufweist (IP19936).

IP28 umschreibt dies mit einer Suchbewegung mit dem Anspruch nachhaltiger Entwicklung, die Wiederentdeckungen ebenfalls einbezieht. Dabei postuliert er, dass Innovationen aus allen gesellschaftlichen Richtungen gefördert werden sollen (IP28 ${ }^{937}$ ). Es soll also von vornherein keine Restriktion geben für das Formulieren von gesellschaftlichen Herausforderungen, wobei die Akteure aufeinander zugehen sollten. Innovationsförderung hat in seinem Verständnis nichts mit Größe zu tun und auch nichts mit hierarchischem Stellenwert im sozialen Gefüge, sondern Innovationsförderung stellt eine gesamtgesellschaftliche Anforderung auf allen Ebenen und in allen Segmenten dar. In der Konsequenz wünscht sich IP28, dass positive Lobbyarbeit von Verbandsstrukturen genauso sichtbar gemacht wird wie kleine ehrenamtliche Initiativen, da ein jeder sein eigener Innovator im Mikrosystem sein kann, mit Auswirkungen nach außen ins Makrosystem. Genauso kann Innovationskraft vom Makrosystem auf das Mikrosystem einwirken. Daraus schlussfolgernd steht er mit seinem Selbstverständnis im direkten Kontakt zum operativen Geschäft, zumal Bewältigungsstrategien bottom-up entstehen, im direkten Kontakt zu operativ Tätigen und Betroffenen, nicht auf Distanz oder top-down (IP28 $8^{938}$; vgl. ebenso $\operatorname{IPp}^{939}$ ). Diese Ausführung steht im Widerspruch zu Fueglistaller et al., die für KE betonen, dass „Entrepreneure (...) am und nicht im Unternehmen arbeiten [sollten]“, um Geschäftsmodellinnovation zu ermöglichen (2019, S. 171).

Zudem lässt sich der Innovationsbegriff durchaus synonym verstehen zur Erweiterung von Zugangsmöglichkeiten, was lediglich einer Wirkungsverbreiterung gleichkommt (IP27 $7^{940}$ ). Ebenfalls kann eine Innovation aus dem Weiterdenken eines bestehenden Sozialunternehmens hervorgehen (IPT ${ }^{941}$ ) und Neuentwicklungen können sich auf andere Produkte oder Branchen übertragen lassen: Dieser Befragte weiß, dass über sein Produkt ein Hochskalieren gar nicht möglich sein kann, da die begrenzten Ressourcen zur Erstellung des Produkts eine deutliche Limitation mit sich bringen. Der SE möchte dennoch seinen sozialen Impact erhöhen und hat dafür zum speziellen Produktionsprozess eine Dienstleistung entwickelt, die ihm ein Hochskalieren ermöglichen kann (IPT ${ }^{942}$ ). 
Eine unverhältnismäßig breit gefasste Begriffsverwendung liegt vor, wenn letztendlich jede Form von Verhaltensänderung als Innovation aufgefasst wird, unabhängig von der Erstmaligkeit oder Wiederentdeckung. Da sich im Leben kontinuierlich neue Situationen ergeben, fehlt es an der notwendigen Trennschärfe: Innovation wird dann zum Synonym für das Sammeln neuer Erfahrungen, was unter wissenschaftlicher Perspektive zur Nutz- und Sinnlosigkeit der Begriffsverwendung führt (vgl. Gillwald, 2000, S. 17 ff.). In diesem Sinn würde z. B. eine Schein-Innovation vorliegen, wenn ein SE zur freiwilligen Selbstkontrolle eine Beiratsstruktur implementiert und diese Veränderung als Innovation deklariert.

Norman (1993) macht eine Verbesserung zur Ausgangssituation zur Bedingung für Innovation, was eine Wirkungsmessung impliziert. Demnach kann unter wissenschaftlichem bzw. qualitätssicherndem Aspekt erst dann von einer Innovation gesprochen werden, wenn die Wirkung nachgewiesen wurde. Die Kriterien für eine Wirkmessung bleiben jedoch unklar. Umso deutlicher wird, dass leichtfertig und oberflächlich zugleich mit dem Innovationsbegriff umgegangen wird.

Außerdem bestehen geteilte Auffassungen dazu, inwieweit soziale Innovation unabhängig davon gegeben ist, wenn sie zu einer Schadwirkung führt (vgl. Rogers, 1983). Hierzu passend verweist Zapf (1989) auf Ogburn, der u. a. den $\mathrm{Ku}-\mathrm{Klux}-\mathrm{Klan}$, also den terroristischen Geheimbund in den Südstaaten der USA, als sozial innovativ auffasst (vgl. Ogburn \& Gilfillan, 1933, S. 162). Demgemäß ist eine positive Konnotation zum Begriffsverständnis von sozialer Innovation grundsätzlich infrage zu stellen. In jedem Fall ist zu beachten, dass auch bei sozialen Innovationen neben ,,gezielten, beabsichtigten, geplanten und vorhersehbaren Wirkungen auch Nebenwirkungen auftreten und nicht beabsichtige, ungeplante und unvorhergesehene Wirkungen möglich sind" (Gillwald, 2000, S. 21). Wie bereits ausgeführt, mangelt es an Wirkanalysen von SEO und dementsprechend an einer differenzierten Auseinandersetzung mit direkten und indirekten Nutzenund Schadeffekten.

Unabhängig davon können Sozialunternehmer erst ,,[d]urch die Verknüpfung von Gemeinwohl und Erwerbswirtschaft (...) als ,sozial innovativ [gelten]““ (Metzger, 2019, S. 1).

In der Gesamtbetrachtung wird deutlich, dass der sehr uneinheitlich und geradezu inflationär verwendete Innovationsbegriff, zudem bei oftmals nicht geklärtem Begriffsverständnis, nicht nur sich widersprechende Erwartungshaltungen wecken kann, was $\mathrm{SE}(\mathrm{O})$ in der Minimalanforderung zu erfüllen haben. Darüber hinaus kann es zu Ausgrenzungen kommen, wenn SE(O) innerhalb des bestehenden verschiedenartigen Anspruchsdenkens bestimmte Vorstellungen nicht erfüllen. Zudem wird der Bedeutungsgehalt von Innovation verwässert, wenn derart vielem in irgendeiner Art und Weise Innovation zugesprochen wird. 


\subsection{Zielsetzung und Zielerreichung}

Bleiben Leistungsziele zu abstrakt (z. B. „Das soziale Problem ist zu lösen“) bzw. werden sie nicht hinreichend operationalisiert (z. B. „Innovation ist Grundvoraussetzung"), dann kann dies zur Unklarheit im Hinblick auf die Zielerreichung führen. Wird hingegen Social Impact im Rahmen einer machbaren Zielsetzung spezifiziert und messbar gestaltet, dann können Leistungserfolge bewusst gemacht, die eigenen Grenzen der Machbarkeit vergegenwärtigt und ein selbst erzeugter Druck zur Wertschöpfung reduziert werden, wie aus der Zielsetzungstheorie (Locke \& Latham, 1990; 2002) hervorgegangene Erkenntnisse belegen.

Die von Locke und Latham entwickelte Zielsetzungstheorie widmet sich im Schwerpunkt der übergeordneten Leitfrage, wie bereits gesetzte Ziele realisiert werden. Als realisierungsorientierte Theorie konzentriert sie sich somit auf die volitionale Bewusstseinslage, die im Nachgang zur realitätsorientierten motivationalen Bewusstseinslage passiert, bei der Ziele erst noch geplant werden und die somit von Intentionsbildung geprägt ist. Die Zielsetzungstheorie grenzt sich insofern ab von den in Kapitel 2 vorgestellten Studienerkenntnissen zur Intentionsbildung und von den Grundüberlegungen Atkinsons zur Risikowahl (1966), die sich ebenso auf prädezisionale motivationale Prozesse beziehen, bei denen Ziele erst noch gesetzt und realitätsorientiert unter Abwägen aller Informationen im Entscheidungsprozess reflektiert werden (vgl. Heckhausen \& Kuhl, 1985).

Goal Setting stellt ein vielseitig untersuchtes Konzept dar. Allerdings ist die Zielsetzungstheorie im Führungskontext entstanden, um zu erforschen, unter welchen Bedingungen Führungskräfte in Unternehmen ihre Mitarbeiter durch Zielsetzungen zu guten Leistungen motivieren können. Somit lassen sich unmittelbare Handlungsempfehlungen für $\mathrm{SE}(\mathrm{O})$ nur unter Vorbehalt aus empirisch belegten Erkenntnissen ableiten.

Im Kern gehen Locke und Latham davon aus, dass herausfordernde Ziele, also Ziele mit einem relativ hohen Schwierigkeitsgrad, gekoppelt mit präzise benannten Zielen, also Zielen mit hoher Spezifität, zu einer höheren Leistung führen als leichte, nicht spezifisch benannte Ziele (ebd.; zur empirischen Absicherung vgl. z. B. Schmidt \& Kleinbeck, 1999; Kleinbeck \& Schmidt, 1996; Brunstein, Schultheiss \& Grässmann, 1998; Schultheiss \& Brunstein, 1999; zu motivkongruenten Zielen vgl. Brunstein et al., 1998; zu Goal Imagery vgl. Schultheiss \& Brunstein, 1999; als Metaanalyse vgl. Miner, 2015). Dennoch sind mehrere Zusammenhänge nicht hinreichend abgeklärt und teilweise liegen inkonsistente Untersuchungsergebnisse vor (zur kritischen Reflexion der empirischen 
Datenlage vgl. z. B. Kanfer, Chen \& Pritchard, 2012; Miner, 2015). Nachfolgend wird nur auf empirisch gesicherte Erkenntnisse eingegangen, die für die vorliegende Untersuchung relevant sind.

Als empirisch gesichert gilt, dass mehrere Moderatoren und Mediatoren den Zusammenhang zwischen schwierigen, spezifischen Zielen und der personenbezogenen Leistung, also der Wirksamkeit der Ziele, beeinflussen. Abbildung 8.5 fasst dazu die nachfolgenden Ausführungen im Schaubild zusammen.

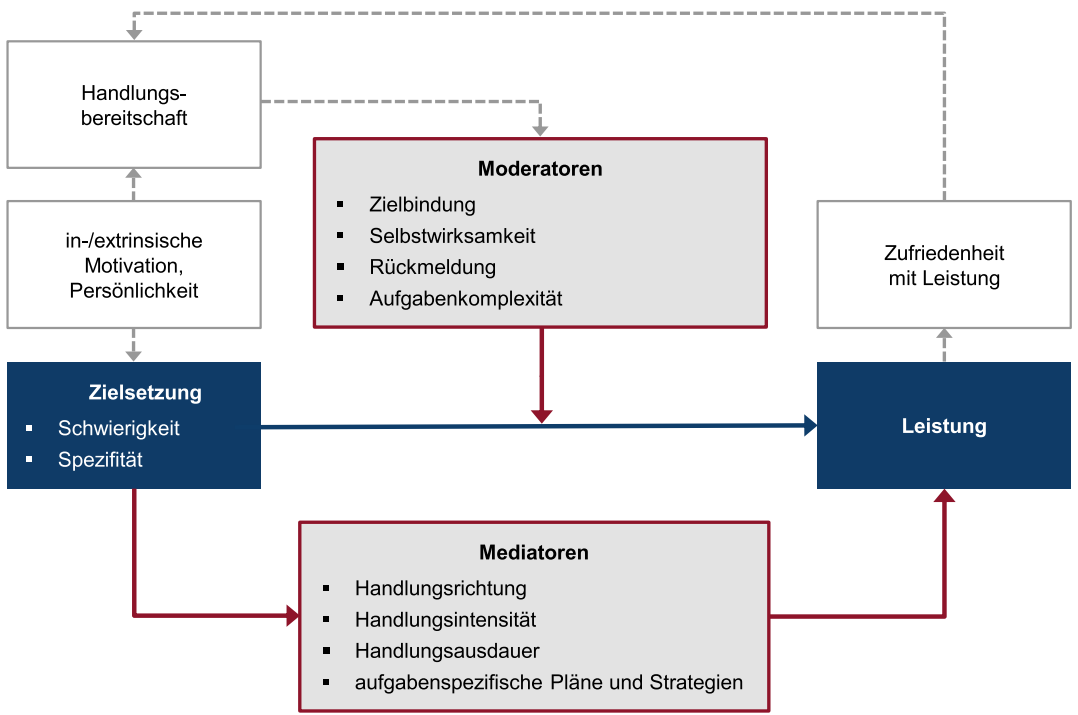

Abbildung 8.5 Moderatoren und Mediatoren der Zielsetzung als Prozessschaubild. (eigene Darstellung)

\subsubsection{Moderatoren zur Zielsetzung}

Bedeutsame Moderatoren sind Zielbindung, Selbstwirksamkeit, Rückmeldung und Komplexität der Aufgabe.

Bei der Zielbindung (Goal Commitment) besteht ein positiver Effekt auf die Leistung, wenn die Notwendigkeit zur Zielsetzung nachvollzogen wird und 
Partizipation bei der Zielfestlegung vorliegt, denn fremdgesetzte und selbstgesetzte Ziele unterscheiden sich in ihrem Anreizwert (Wagner, 1994). Eine affektiv starke Verbundenheit mit einem Ziel begünstigt das Leistungsverhalten (Klein, Wesson, Hollenbeck \& Alge, 1999). Weitere Motivatoren können sich begünstigend auf die Zielbindung auswirken. Wie weiter oben bereits skizziert wurde und in Abschnitt 8.12 noch vertiefend betrachtet wird, geben Befragte zu verstehen, affektiv und deutlich stärker mit der sozialen Zielsetzung verbunden zu sein als mit der unternehmerischen Zielsetzung. Geschildert wird zudem, dass gelingende Netzwerkarbeit auf Goal Commitment positiv einwirken kann $\left(\mathrm{IPG}^{943}\right)$.

Hinsichtlich Selbstwirksamkeit (Bandura, 1997) besteht ein positiver Effekt sowohl auf die Zielbindung als auch auf die Leistung, wenn aufgabenspezifisches Selbstvertrauen gegeben ist (Locke \& Latham, 2002). Demnach nehmen Erwartungs- und Überzeugungsmuster zu Konsequenzen einer Handlung Einfluss darauf, inwieweit eine Person ihrer angedachten Handlung nachkommt (vgl. Bandura \& Locke, 2003). Befragte mit ausgeprägtem Selbstvertrauen im Hinblick auf das Bewältigen einer Aufgabe, inklusive einer hohen Bewertung ihres Fleißes, geben zu verstehen, dazu zu neigen, ein höheres Maß an Ausdauer zum Erreichen ihrer Zielsetzung zu entwickeln (IP2 ${ }^{944}$, i. V. m. IP2 ${ }^{945}$ ). Dabei kann ein intensiveres Gefühl der Verbundenheit gegenüber herausfordernden Zielen gegeben sein. Ebenso kann ausgeprägte Selbstwirksamkeit begünstigen, negative Kritik konstruktiv zu verarbeiten und als Ansporn für ein besseres Bewältigen von Aufgabenanforderungen zu wirken, einhergehend mit der Wahl effektiverer Verfahrenswege zur Zielerreichung (Seijts \& Latham, 2001; zur internationalen SE-Forschung vgl. hierzu Clark, Newbert \& Quigley, 2018). Allerdings ist davon auszugehen, dass sehr hohe Selbstwirksamkeitsüberzeugung in Selbstüberschätzung münden kann und damit zur Fehlbelastung wird (vgl. Vancouver, Thompson, Tischner \& Putka, 2002). Erinnert wird beispielhaft an IP13, der sich auffallend selbstwirksam erlebt, selbstbewusst hochskaliert (IP13 ${ }^{946}$, i. V. m. IP13 ${ }^{947}$ ) und dabei seine Abhängigkeiten ignoriert (IP13 ${ }^{948}$ ), was in eine akute Bedrohung von Insolvenz eskalierte.

SE sollten daher partizipativ in die Vereinbarung von Zielen eingebunden werden bzw. den Sinn in der Zielsetzung nachvollziehen und mittragen können, da sich dies positiv auf deren Selbstwirksamkeit und damit auf die Zielbindung auswirken kann. Befragte zeigen auf, z. B. in Förderkontexten mit stark vorgegebenen Zielen konfrontiert zu werden (IP29 ${ }^{949}$; IP1 ${ }^{950}$ ), wodurch sie sich weniger selbstwirksam erleben, was in der Folge der Zielbindung entgegenwirkt. Ebenso können Kompetenzmängel von hinzugezogenen Experten Einfluss nehmen auf 
die eigene Selbstwirksamkeit (IP28 ${ }^{951}$ ). Dabei können SE mit hoher Selbstwirksamkeitsüberzeugung Stresssituationen wie z. B. Rückschläge besser meistern (IP25 $5^{952}$ ).

Bei Rückmeldungen zu (Teil-)Zielen besteht ein positiver Effekt auf das Leistungsverhalten, wenn die Ziele zuvor definiert worden sind und wenn das Feedback regelmäßig erfolgt. Feedback zu nicht klar vereinbarten Zielen erwirkt keinen leistungssteigernden Effekt (Schmidt, 2010). Darüber hinaus sollte sich eine Rückmeldung nicht nur auf Ergebnisse, sondern auf das Zustandekommen von Ergebnissen und somit auf Prozesse und hierbei ebenso auf Teilerfolge wie auf Defizite in der Teilzielerreichung beziehen (z. B. Earley, 1988), was zudem strategische Anpassungen ermöglicht. Zur Sicherung der Zielerreichung sollten personengebundene negative Rückmeldungen zu deren Eigenschaften nicht dazu führen, dass die Person fortan weniger die Aufgabe als vielmehr ihre eigene Person fokussiert, um hierdurch bedingte Einbußen auf die Performanz zu vermeiden (Kluger \& DeNisi, 1996). Dabei zeigen Befragte auf: Ein Feedback zu Defiziten in der Zielerreichung zieht dann eine Leistungssteigerung nach sich, wenn der Feedback-Empfänger selbst unzufrieden mit der Ist-Situation ist und wenn er eine ausgeprägte Selbstwirksamkeits- und Kontrollüberzeugung hat, einhergehend mit dem Bedürfnis, seine Leistung zu steigern (IP6 ${ }^{953}$ ). Die kontinuierlich erfolgenden Rückmeldungen sollten somit konstruktiv sein, wobei ein informeller Weg die positive Wirkung begünstigen kann.

Um Lernprozesse zu ermöglichen, sollte das Feedback zur Zielerreichung in Reflexionsphasen genutzt werden, was Flexibilität und Veränderungsbereitschaft in der Zielsetzung voraussetzt. Mit den Anpassungsprozessen lassen sich über die ursprünglich festgesetzten Ziele modifizierte Zielsetzungen bestimmen. Hierzu passend begründet Luhmann $(1984 ; 2011)$ systemtheoretisch, dass die Fähigkeit zur Anpassung an sich verändernde Umwelteinflüsse für alle Organisationen überlebensnotwendig ist, wobei Selbstorganisation als natürliche Eigenschaft eines sozialen Systems zu verstehen ist. Denn Systeme grenzen sich bereits mit ihrem eigenen Schaffen (Autopoiesis) gegenüber ihrer Umwelt ab, so dass sie in sich operativ geschlossen sind. Dies bestätigen Befragte für ihre SEO-Gründung (IP18 ${ }^{954}$; i. V. m. IP18 ${ }^{955}$ ). Auf der Innenseite der System-Umwelt-Grenze kommt es dabei durch das einseitige Gestalten von Prozessen zu einer selektiven Umweltwahrnehmung; Systeme können sich nur auf ihre systemimmanenten Operationen beziehen (Selbstreferenz). Würden Systeme ihre Gestaltung in der Umwelt vornehmen, dann würden sie ihre Abgrenzung aufgeben und damit sich selbst auflösen. In der Folge kann es sich als klug erweisen, bei der Überprüfung und Anpassung von Zielsetzungen sowie bei den daraus hervorgehenden Managementansätzen Impulsgebern aus der Umwelt Zutritt zum System zu gewähren 
und deren Feedback sowie Maßnahmenvorschläge ganz im Sinne einer systemerweiterten Betrachtung einzubeziehen. Vereinzelt implementieren Befragte Board-Gremien für diese Lernprozesse (IPU ${ }^{956}$ ). Dabei ist auf die Zusammensetzung der Feedbackgeber zu achten und ebenso darauf, dass Perspektivwechsel vorgenommen werden können (IP28 ${ }^{957}$; IP28 ${ }^{958}$ ). Dies bezieht sich ebenfalls auf SE, die ihre SEO im Team führen und die demzufolge nicht nur gut zusammenarbeiten können müssen, sondern darüber hinaus ihre Performanz aus verschiedenen Blickwinkeln und im Hinblick auf Interessen unterschiedlicher Stakeholder reflektieren, um dem Group-Think-Phänomen vorbeugen zu können. Dabei kann eine zu spezifische Zielsetzung zum Scheuklappendenken führen und in der Folge Perspektivwechsel hemmen.

Kooperationen, die in ihrer interdisziplinären Zusammenarbeit eine konstruktive Feedback-Kultur umsetzen, können demzufolge Risiken besser kalkulieren und nicht kalkulierbare Situationen, also Ungewissheit, die bei zunehmender Aufgabenkomplexität umso wahrscheinlicher wird, besser managen (Norman \& Shimer, 1994).

Aufgabenkomplexität nimmt ebenfalls Einfluss auf den positiven Zusammenhang zwischen herausfordernden, spezifischen Zielen und der Arbeitsleistung. Dieser positive Zusammenhang ist allerdings bei überschaubaren Aufgaben deutlich stärker ausgeprägt (Locke \& Latham, 2002). Zu komplexe Aufgaben können die Leistung beeinträchtigen, da der Koordinationsaufwand überhandnehmen kann und zudem Veränderungen im Arbeitsprozess wahrscheinlicher sind (Wood, Mento \& Locke, 1987; Kleinbeck \& Schmidt, 2004). Befragte heben hierzu passend hervor, dass komplexe Aufgaben eine gewissenhafte Problemanalyse benötigen (IPT ${ }^{959}$; IPR ${ }^{960}$ ), einhergehend mit strategischem Planungsmanagement, um den positiven Leistungseffekt erzielen zu können. Zudem muss sich die Aufgabenkomplexität an der Qualifikation und an den Kompetenzen der betreffenden Person orientieren (IP $\psi^{961}$ ).

Mit zunehmender Komplexität von Zielsetzungen kann eine Kalkulation zur Risikoeinschätzung und somit das Management von Risiken verhindert werden und sich stattdessen ein strategischer Umgang mit Unsicherheit als sinnvoll erweisen (IP8 ${ }^{962}$ ). Umso wichtiger erscheint es hierbei, bei komplexen Zielsetzungen die zur Bewältigung erforderlichen Kompetenzen definieren zu können und sicherzustellen, dass diese Kompetenzen abrufbar sind bzw. erworben werden können. Dabei können sich spezifische Lernziele, die auf Kompetenzerweiterung abzielen und den Handlungsprozess reflektieren, leistungssteigernd auswirken (Winters \& Latham, 1996; vgl. auch Latham, Seijts \& Crim, 2008; IP26 $6^{963}$, i. V. m. IP26 $6^{964}$ ).

Teilziele und Meilensteine sollten immer dem übergeordneten Gesamtziel sowohl des SE als auch der SEO zugeordnet werden können. Beispielsweise 
beschreibt ein Befragter eine übergeordnete Zielsetzung, aus der er spezifische Meilensteine ableitet $\left(\operatorname{IPp}^{965}\right)$.

\subsubsection{Mediatoren zur Zielsetzung}

Anspruchsniveau und Spezifität einer Zielsetzung wirken unmittelbar auf mehrere motivierende, intrapersonelle Wirkmechanismen (zum volitionalen Bedeutungsgehalt der Handlungsintensität und -dauer vgl. z. B. Earley, Wojnaroski \& Prest, 1987, S. 107). Zu diesen Mediatoren zählen die Handlungsrichtung, Handlungsintensität und Handlungsausdauer sowie die Abrufbarkeit probater bzw. geeigneter aufgabenspezifischer Pläne und Strategien.

Die Handlungsrichtung beschreibt die Steuerung der Aufmerksamkeit von Teilaspekten einer Aufgabe. Spezifische Zielvorgaben provozieren die Suche nach Informationen, die relevant für die Zielerreichung sind. Unspezifische Zielvorgaben hingegen erhöhen die Wahrscheinlichkeit, dass für die Zielerreichung relevante Informationen ausgeblendet werden. Allerdings besteht bei hoher Zielspezifität die Gefahr, einseitig zu fokussieren und dabei wichtige Aspekte $\mathrm{zu}$ vernachlässigen und beispielsweise das Unternehmerische nicht mehr einzubeziehen. Befragte beschrieben, dass sie operativ umtriebig und engagiert im Hinblick auf die soziale Zielerreichung agieren, wenn sie unternehmerische Aspekte ausblenden (gemäß der Ausführung in Abschn. 8.3; siehe ebenso die Diskussion zur konzeptionellen Unvereinbarkeit von Sozialem und Unternehmerischem in Abschn. 8.12).

Kommt es zu Anpassungsprozessen bei der Handlungsausrichtung, ist der Grundhaltung im Sinne des sozialunternehmerischen Wertegerüsts treu zu bleiben. Ein Geschäftsmodell muss sich dabei nicht verändern, aber eventuell das Wirkungsmodell. Anknüpfend an die Ausführung in Abschnitt 8.9 zum Anspruchsniveau der Innovationsentwicklung wird betont, dass ein Anpassungsprozess bei der Handlungsausrichtung zwar einen innovativen Weg darstellen kann, dies aber keinesfalls gegeben sein muss. Denn das Ziel ist nicht die Innovation, sondern z. B. nachhaltige Entwicklung zu ermöglichen. Ob Innovation dabei als Mittel zum Zweck einen denkbaren Weg zur Zielerreichung darstellt, ist demzufolge zweitrangig und in keinem Fall obligatorisch. Nicht das Neue ist wegweisend für die Handlungsrichtung, sondern die veränderte Wirkung.

Die Handlungsintensität beschreibt bei der Zielerreichung die Anstrengung zur Ressourcenmobilisierung. Dabei besteht ein Zusammenhang zwischen handlungsbezogener Anstrengung und Aufgabenschwierigkeit (Locke, 1975; Locke \& Shaw, 1984). Der zugrundeliegende Anpassungsprozess des Verhaltens an die 
Situation wurde erstmals untersucht von Ach (1910), der bei willenspsychologischen Experimenten den Einfluss von Zielvorstellungen bei Handlungsabläufen einbezogen hat und mit dem daraus hervorgegangenen ,Schwierigkeitsgesetz der Motivation" aufzeigt, dass die Willenskraft zur Zielerreichung und Aufmerksamkeitskonzentration bei höheren Hindernissen bzw. bei schwierigeren Aufgaben stärker ausgeprägt ist als bei niedrigen Hindernissen bzw. bei leichteren Aufgaben. Selbstverständlich sind im Gesamtgefüge die weiteren zielsetzungstheoretischen Erkenntnisse, beispielsweise die dargestellten Besonderheiten der Aufgabenkomplexität, zu berücksichtigen.

Allerdings kann die Leistung leiden, wenn eine - beispielsweise betriebswirtschaftliche - Aufgabe mit einem ausgeprägten inneren Widerstand versehen ist, sie als unliebsam empfunden oder als zu hohe Anstrengung bewertet wird, eine nicht mehr zu bewältigende Hürde darstellt und in der Folge eine Überforderungssituation eintritt, die zur Vermeidungshaltung führt (vgl. Abschn. $8.3 \mathrm{mit}$ Abb. 8.3).

Spezifische, anspruchsvolle Ziele führen zu einer vergleichsweise hohen Handlungsausdauer. Bei zeitlichem Druck besteht jedoch u. a. die Gefahr, dass eine quantitative Zielverfolgung auf Kosten von Qualität erfolgt. Mit diesem Kompensationsmechanismus lässt sich zwar Leistungsfähigkeit erhalten, aber Betroffene geraten dabei an ihre Belastungsgrenzen (IP25 ${ }^{966}$ ). Ebenso kann Zeitdruck zur Verunsicherung führen zulasten der eigenen Selbstwirksamkeitsüberzeugung (IP24 ${ }^{967}$ ). Zeit- und Ergebnisdruck können zudem das Begehen von Regelverstößen und unethische Verhaltensweisen provozieren (Schweitzer, Ordóñez \& Douma, 2004).

Zudem benötigen Betroffene zur Aufrechterhaltung ihrer Handlungsausdauer das Wissen über die Notwendigkeit von Aufgaben und die Kompetenz, diese Aufgaben zu bewältigen. SE, die das Unternehmerische mit eigenen Kompetenzschwächen und somit mit nicht gut bewältigbaren Hürden verankern, benötigen daher Beratung und Begleitung bei der Anwendung und nicht nur ein theoretisches Training im Vorfeld (IPG ${ }^{968}$ ).

Darüber hinaus erweist sich die Verfügbarkeit von machbaren aufgabenspezifischen Plänen und Strategien als bedeutsam, insbesondere zur Bewältigung komplexer Ziele. Gerade probate Pläne erweisen sich als hilfreich für weitere Verwendungen. Allerdings müssen für neue und vor allem für sehr komplexe Aufgaben entsprechende Planungsschritte und strategische Ausarbeitungen vorgenommen werden, was einen Arbeitsaufwand darstellt und kurzfristig gedacht das Leistungsvermögen reduzieren kann, der sich aber amortisieren kann. 
SE, die ihren Markt erst erobern müssen und auf Förderung angewiesen sind, berichten, dass sie keine Mittel für das notwendige Entwickeln aufgabenspezifischer Arbeitsprozesse bereitgestellt bekommen (vgl. Abschn. 6.1). Fehlende Projekt- und Prozessmanagementkompetenzen können somit nicht eingekauft werden.

Kapitelabschließend ist zu den zielsetzungstheoretischen Ausführungen ergänzend kritisch anzumerken:

- Spezifische, anspruchsvolle Ziele können das Erfahrungslernen hemmen, weil die Bereitschaft sinken kann, aufgabenbezogene Handlungsalternativen auszuprobieren (vgl. Ordóñez, Schweitzer, Galinsky \& Bazerman, 2009). Dabei können spezifische Ziele als relativ geringwertig bzw. bedeutungsarm erlebt werden (Little, 1989) - im Gegensatz zu sehr weit gefassten Zielsetzungen, die das Erbringen zahlreicher Handlungen und Tätigkeiten über einen längeren Zeitraum einfordern (Emmons, 1992).

- Somit ist situationsbezogen abzuwägen, inwieweit sich bei einer spezifischen Zielsetzung ein Präzisieren von Unterzielen erübrigt, da sich diese Zielsetzung in genaue Handlungsabläufe herunterbrechen lässt. Ebenso gilt es zu bedenken, dass sich bei weit gefassten Zielsetzungen, die bei zunehmendem Abstraktionsniveau eine Grundhaltung verkörpern, eventuell konkrete Handlungen nicht mehr herleiten lassen. Es erscheint sinnvoll, dies kontinuierlich auf Machbarkeit hin zu überprüfen.

- Die aus der Zielsetzungstheorie hervorgegangenen Erkenntnisse liefern keine Antwort auf die Frage, inwieweit Intentionsbildungsprozesse, die zusätzlich $\mathrm{zu}$ bereits bestehenden Intentionsbildungsprozessen z. B. infolge neuer, unerwarteter Anforderungen aus der Umwelt in Erscheinung treten, volitional einwirken. Die isolierte Betrachtung einzelner Intentionsbildungsprozesse im Phasenablauf wird der dynamischen wie komplexen Realität nicht gerecht. Neben- und Wechselwirkungen von Erfahrungswerten aus der postaktionalen Bewertungsphase, beispielsweise als Erfolgserlebnisse im Unternehmerischen, bleiben hierbei ebenfalls unberücksichtigt.

In der Gesamtbetrachtung wird deutlich, dass SE durch definitorische Vorgaben nicht mit Problemlösungsansprüchen konfrontiert werden sollten, schon gar nicht unter unverhältnismäßig hohen Leistungsdruck gesetzt werden sollten und sich auch selbst nicht mit diesem Anspruchsdenken brandmarken sollten. Stattdessen sollten sie sich Ziele nach den zuvor ausgeführten Kriterien setzen und diese im kontinuierlichen Überprüfungsprozess hinsichtlich ihrer Erreichbarkeit und Realisierbarkeit bewerten, so dass ein SE sich und seine SEO hierauf bezogen in 
spezifischer Form bewerten kann. So kann IP14 zwar ebenfalls, wie auch alle anderen Befragten, das soziale Problem nicht lösen, dem er sich im Rahmen seiner SEO-Arbeit widmet (IP14 ${ }^{969}$; siehe ebenso die Ausführung in Abschn. 8.8 zum Skalierungsanspruch dieser SEO). Aber er erreicht mit seiner SEO-Arbeit seine Zielgruppe i. S. v. Wirkungsempfängern, hierbei sein spezifisch gesetztes Ziel $\left(\right.$ IP14 ${ }^{970}$ ). Dabei richtet er sowohl die Möglichkeit und Notwendigkeit eines Hochskalierens als auch die Notwendigkeit des Runterskalierens nach den gegebenen Rahmenbedingungen, die er selbst nicht zu verantworten hat, bestmöglich aus. Seine Verantwortung sieht er $u$. a. darin, auf Umweltveränderungen mit unternehmerischen Mitteln zum Schutz der SEO zu reagieren, ohne möglichst die soziale Wirkung dabei zu schmälern, wobei er ausgesprochen erfolgreich ist.

\subsection{Stellenwert des Unternehmertums im Verhältnis zur sozialen Zielsetzung}

Befragte benennen ihr unternehmerisches (Selbst-)Verständnis bzw. die unternehmerische Ausrichtung ihrer SEO in unterschiedlicher Ausprägung und Gewichtung: von fundamental verankert und zentral bedeutsam (IP35 $5^{971}$; IP19 ${ }^{972}$; IP25 ${ }^{973}$ ) über gleichwertig zum sozialen Sinn (IPF ${ }^{974}$; IPA ${ }^{975}$; IPk ${ }^{976}$ ) bis zur Begleiterscheinung der zentral bedeutsamen sozialen Zielsetzung (IPÖ ${ }^{977}$ ). Ebenso kann ein hoher Bedeutungsgehalt des Finanziellen in Relation zur sozialen Mission der SEO bewertet werden (IP23 ${ }^{978}$ ). Zudem kann sich das unternehmerische Denken und Handeln in Abhängigkeit von der persönlichen Zielsetzung (IP19979) bzw. der Bereitschaft und Haltungsflexibilität verändern

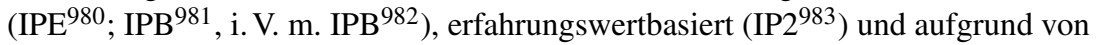
Handlungsdruck aus der Umwelt, also reaktiv aus einer Not heraus (IP14 ${ }^{984}$ ). Dabei können Veränderungsprozesse zu Rechtfertigungsdruck führen (IP26 ${ }^{985}$ ). Ebenso ist denkbar, dass das Entwickeln einer unternehmerischen Haltung nicht zwangsläufig zur Identifikation mit Unternehmertum führt (IP7 ${ }^{986}$ ).

Eine IP priorisiert das Unternehmerische höher als das Soziale bzw. als Grundvoraussetzung: Soziale Zielsetzung hat sich demnach zu unterwerfen und geht aus dem Unternehmerischen hervor (IP1 ${ }^{987}$ ). Allerdings äußert sich diese IP in der inhaltlichen Ausführung hierzu auffallend widersprüchlich. Somit kann auch in diesem Fall lediglich festgehalten werden, dass ein Ungleichgewicht besteht, also das Soziale und das Unternehmerische nicht gleichwertig betrachtet werden.

Einige Befragte geben zu verstehen, dass sie zwischen Sozialunternehmertum und konventionellem Unternehmertum keine relevanten Unterscheidungsmerkmale sehen $\left(\mathrm{IPB}^{988} ; \mathrm{IP}^{989}\right.$ ). Sozialunternehmertum kann demnach als normales 
Unternehmertum aufgefasst werden mit einem „Zusatzmodul“, das sich nicht im Skill-Set zeigt, sondern in der Haltung und im Anspruchsdenken $\left(\mathrm{IPb}^{990}\right)$. Insofern kann als Hauptmerkmal von Social Entrepreneurship angesehen werden, das Generieren von Einnahmen mit der Erfüllung einer sozialen Mission zu kombinieren (Mair \& Martí, 2006; Bacq \& Janssen, 2011). Bei Befragten, die „Social“ und „Entrepreneurship“ harmonisch miteinander verbinden bzw. diesen Anspruch sich selbst gegenüber bekunden, sind ebenso SEO mit Gemeinnützigkeitsstatus vertreten, die auf Fördermittel angewiesen sind, da ihre Kunden nicht bereit bzw. nicht in der Lage sind, eine Bezahlung zu leisten (IP ${ }^{991}$ ). Allerdings wird SEOSupport durch Stiftungen zuweilen als Kontraindikator für sozialunternehmerische Wirkung angesehen (IPO ${ }^{992}$ ).

Bei befragten SE, die das Unternehmerische als Begleiterscheinung der zentral bedeutsamen sozialen Zielsetzung bewerten, wird die wirtschaftliche Komponente vernachlässigt (IP3 ${ }^{993}$; IPV ${ }^{994}$ ); im Zielkonflikt zwischen der gemeinwohlorientierten Zielsetzung und der finanziellen Absicherung kann sich der SE für die soziale Zielsetzung entscheiden $\left(\operatorname{IP}^{995}\right)$, was als fundamentales Merkmal für SE diskutiert wird (z. B. bei Rummel, 2011, S. 73). Demgemäß unterscheiden sich SE von KE in der Mission, die ihre Aktivitäten leitet (Austin, Stevenson \& Wei-Skillern, 2006; Neck, Brush \& Allen, 2009).

Eventuell verleihen Befragte dem Unternehmerischen gar kein Stellenwert im eigenen Konzeptverständnis oder in deutlich untergeordneter Funktion (IP14 ${ }^{996}$; IP $\ddot{A}^{997}$ ). Ggf. werden lediglich Management-Elemente in die SEO integriert (IP15 ${ }^{998}$; vergleichbar mit Scharpe \& Wunsch, 2020, S. 11), was zudem nicht von Anfang an geschehen muss, sondern sich im Laufe der Zeit entwickeln kann $\left(\right.$ IP16 $6^{999}$ ). Dabei muss keine Anforderung an eine Organisationsform gestellt werden, bei der das Unternehmerische klar zum Tragen kommt, insbesondere, wenn finanzieller Profit dem sozialen Mehrwert untergeordnet wird und hierbei keine gleichberechtigte Verbindung, geschweige denn eine miteinander verschmolzene Einheit entsteht (IPC ${ }^{1000}$; IP17 ${ }^{1001}$, i. V. m. IP17 ${ }^{1002}$; ebenso z. B. bei Harbrecht, 2010, S. 73). Beispielsweise scheiterte IP17 mit ihrer SEO am Markt und zeigte sich zuvor beratungsresistent zu finanziellen Fragen, klammerte bei ihrer Betrachtung von Nachhaltigkeit die ökonomische Komponente geradezu aus (IP17 $7^{1003}$, i. V. m. IP $17^{1004}$, i. V. m. IP $17^{1005}$ ). IP17 ließ sich dabei vom Freundeskreis leiten: Ihr soziales Umfeld wirkte als Filterblase und damit ausschlaggebend für Entscheidungsfindungen, die das Finanzielle ausklammerten. Auf diese Weise sind unternehmerische Fehlentscheidungen entstanden (IP17 ${ }^{1006}$ ).

Dementsprechend besteht bei Gründungen im Team unter Gleichgesinnten, die das Unternehmerische zugunsten der sozialen Wirkung vernachlässigen, die Gefahr, infolge von Group Think nicht auf eine unternehmerische Relevanz 
zu achten (IPJ ${ }^{1007}$; als Positivbeispiel z. B. bei $\left.\operatorname{IP}_{\varepsilon}^{1008}\right)$. Hierbei ist es möglich, dass unternehmerische Wirkung i. S. v. wirtschaftlicher Profitabilität nicht im Anspruchsdenken vorkommt und demzufolge nicht reflektiert wird. Erkennt jedoch ein SE als Einzelkämpfer eigene Defizite, z. B. im betriebswirtschaftlichen Verständnis, kann zu deren Bewältigung das strategisch ausgerichtete gemeinschaftliche Managen einer SEO im Leitungsteam einen geeigneten Lösungsansatz darstellen. Dies kann sowohl reaktiv erfolgen, also durch das Sammeln von Negativerfahrungen nach einer SEO-Gründung (IP6 $6^{1009}$, i.V. m. IP6 $6^{1010}$ ), als auch im eigenen Anspruchsdenken proaktiv (IP8 ${ }^{1011}$, i. V. m. IP8 ${ }^{1012}$ ). Beispielsweise kann dann vermieden werden, dass Defizite im betriebswirtschaftlichen Verständnis zur Romantisierung des Zahlenwerks führen, bei dem ein naives Wunschdenken oder Verdrängung die unternehmerische Basis widerspiegelt $\left(\mathrm{IP} \pi^{1013}\right.$; IP15 ${ }^{1014}$; IP9 ${ }^{1015}$, i. V. m. IP9 ${ }^{1016}$; m. V. a. Abschn. 7.4). Ein unternehmerisches Risikomanagement besteht demgemäß nicht darin, die Bereitschaft zu ermitteln, ein nicht präzisiertes Risiko einzugehen, sondern das Risiko ist zuvor zu kalkulieren. Ansonsten würde Ungewissheit zu managen sein.

In diesem Sinn spendete IP17, die mit ihrer am Markt später gescheiterten SEO zuvor u. a. benachteiligte Arbeiter in einem Drittweltland unterstützen wollte, einen Teil des Umsatzes als Bestandteil ihres Geschäftsmodells (IP17 ${ }^{1017}$ ). Empfänger der Spenden waren gewerkschaftlich organisierte Einzelpersonen im Drittweltland, die IP17 nicht persönlich kannte. Einen Nachweis zum Umgang mit der Spende forderte IP17 grundsätzlich nicht ein. Zumal das Drittweltland auf dem Korruptionsindex von Transparency International einen der untersten Ränge belegt, besteht ohnehin die Gefahr, dass die Unternehmensspenden nicht den ausgebeuteten Arbeitern direkt zugutegekommen sind. Wenngleich der Ansatz im Sinne der Förderung nachhaltiger Entwicklungsstrukturen nachvollziehbar erscheint, dass Betroffene sich selbst emanzipieren sollen, lassen sich der Verbleib und die Wirkung der Unternehmensspenden nicht nachprüfen.

Denkbar ist, dass sich das sozialunternehmerische Konzeptverständnis im Hinblick auf wirtschaftliche Profitabilität darauf begrenzt, nicht defizitär zu wirtschaften $\left(\mathrm{IP} \pi^{1018}\right.$; IPp $\left.{ }^{1019}\right)$. Dabei arbeitet Schwingenstein für ihre Befragten heraus, dass diese die eigene Auseinandersetzung mit der wirtschaftlichen Profitabilität ihrer SEO von sich weisen, wenn ihnen in diesem Zusammenhang ein negatives Unternehmerimage unterstellt wird (2013, S. 87). Somit kommt es für SE, deren sozialunternehmerisches Engagement gesellschaftlich keine Schadwirkung mit sich bringt, sondern ausschließlich zum Vorteil aller direkt und indirekt Beteiligten wird, nicht in Betracht, wirtschaftlichen Profit als Win-win-Situation zu bewerten; dies setzt zudem die innere Haltung voraus, Einkünfte aus dem sozialunternehmerischen Engagement für den eigenen Lebensunterhalt verwenden 
zu dürfen (vgl. Abschn. 8.4). Das aus dem unternehmerischen Agieren hervorgehende Bestreiten des Lebensunterhalts aller Mitarbeitenden und des SE kann dabei als unmittelbarer ökonomischer Effekt aufgezeigt werden. Es können Indikatoren der Wirksamkeit angelegt werden wie z. B. die Höhe und Regelmäßigkeit des Einkommens und die Ausprägung von Altersversorgungsstrukturen. Auf diese Weise wird der ökonomische Erfolg sichtbar und messbar.

Allerdings kann sich bei SE, die den gesellschaftlichen Mehrwert und somit die am Gemeinwohl orientierte Wertschöpfung hervorheben (IP6 ${ }^{1020}$; IP34 ${ }^{1021}$; $\mathrm{IPK}^{1022}$ ), eine andere Konsequenz ergeben: erwirtschaftete Gewinne in das Verfolgen der sozialen Zielsetzung bzw. in ihre nachhaltigkeitsorientierte Unternehmensentwicklung zu reinvestieren (IP2 ${ }^{1023}$; vgl. z. B. Rummel, 2011; Beckmann, 2011). So ,steht die Reinvestition dieser [Gewinne] als Priorität über der Ausschüttung an eventuelle Shareholder. Auf diesem Weg wird ein unfreiwilliger Mission-Drift verhindert" (Osbelt, 2019; wortgleich in Scharpe \& Wunsch, 2020, S. 12; Hoffmann, Scharpe \& Wunsch, 2021, S. 15). Dass das normative Anspruchsdenken, reinvestieren zu müssen, SE im Hinblick auf ihre eigene Absicherung unter Druck setzen kann, wurde im Rahmen der Positionierung gegenüber dem Geldverdienen bereits aufgezeigt.

Hierbei besteht ein weiterer ökonomischer Effekt, der sich auf eine gesellschaftsbezogene Kostenreduktion bzw. Folgekostenersparnis bezieht. Zur argumentativen Untermauerung wird erinnert an die Ausführungen in Abschnitt 5.10, wenn Einsparungen von Steuergeldern, die einzig dem sozialunternehmerischen Engagement zu verdanken sind, außer Acht gelassen werden.

Ebenso wird an die Diskussion erinnert, wenn Social Entrepreneurship zu einem Projekt herabgewürdigt wird (IPü $\left.{ }^{1024} ; \mathrm{IPH}^{1025}\right)$, was bereits bei einer nicht reflektierten Abgrenzung im Begriffsverständnis passieren kann (IPE ${ }^{1026}$; $\mathrm{IPä}^{1027}$ ). Allerdings können ebenso Förderauflagen einen angestrebten Strukturaufbau verbieten, wenn Vorgaben bestehen, dass sämtliche Fördermittel zweckund fristgebunden ausgegeben werden müssen. In der Folge kann aus einem Projekt kein Unternehmen werden, was einen Widerspruch zu den Versprechungen der Politik darstellt (IPÖ ${ }^{1028}$ ). Umso bedeutsamer kann es dabei erscheinen, einen geeigneten Träger als Starthilfe zu haben (IP2 ${ }^{1029}$ ), ggf. als Intrapreneurship (IPE ${ }^{1030}$ ), wobei eine Trennung vom Träger geschildert wird, wenn der SE mit seiner SEO auf eigenen unternehmerischen Füßen stehen kann (IPE $\left.{ }^{1031}\right)$. Gleichwohl kann unternehmerisches Denken und Handeln aus einem sozialen Projekt hervorgegangen sein (IP16 ${ }^{1032}$; $\operatorname{IP} \tau^{1033}$ ), so dass vergleichbar zu einem Entpuppungsprozess eine neue Gestalt aus dem Projekt entsteht, die eine andere Wertigkeit und auch andere Funktionen mit sich bringt und 
Managementstrukturen benötigt (IP34 $\left.{ }^{1034}\right)$. Dabei können aus dem Unternehmen abermals neue Projekte als Bestandteil des Unternehmens mit Produkt- oder Dienstleistungsentwicklungen hervorgehen (IP7 ${ }^{1035}$ ).

Wenn die sozialunternehmerische Basis bzw. die Mission und Vision sich auf das Soziale konzentriert und die unternehmerische Komponente lediglich das Mittel zur Umsetzung darstellt, dann beinhaltet das Gesamtkonstrukt bereits in der Argumentation keine gleichwertige unternehmerische Basis. Dementsprechend kann die Aussage, ein SE setzt seine Mission unternehmerisch um, eine andere und unternehmerisch schwächere sein, als wenn die Aussage lautet, dass ein Unternehmer eine soziale sowie eine wirtschaftliche Zielsetzung gleichbedeutend verfolgt und in diesem Verständnis der SE eine spezielle Form von Unternehmertum repräsentiert sowie aktiv am Marktgeschehen teilnimmt (IP6 ${ }^{1036}$; IP25 ${ }^{1037}$ ). Bei dieser Betrachtung lässt sich Sozialunternehmertum von Unternehmertum nicht abgrenzen, sondern es stellt eine Eingrenzung innerhalb der Vielfalt dar, unternehmerisch aktiv zu sein.

Befragte bekräftigen, dass eine SEO obligatorisch an finanzielle Rendite bzw. Gewinnorientierung gekoppelt ist (IP23 ${ }^{1038}$; IP19 ${ }^{1039}$ ), ein SocialBusiness-Modell abbildet (IP $\xi^{1040}$; IP9 ${ }^{1041}$ ) und demzufolge betriebswirtschaftliche Mechanismen integriert (IPT ${ }^{1042}$ ) bzw. zu integrieren hat (IPF ${ }^{1043} ; \mathrm{IP}^{1044}$ ). Dabei fällt auf, dass Befragte eine Abgrenzung gegenüber monetärer Gewinnmaximierung zugunsten von Gewinnorientierung vornehmen. Denn das Anspruchsdenken gegenüber monetärer Gewinnmaximierung wird von nahezu keinem Befragten benannt und wenn, dann nur sehr untergeordnet (IP17 ${ }^{1045}$ ) und negativ attribuiert (IP2 $\left.{ }^{1046}\right)$.

IP28 erläutert hierbei, dass in seiner SEO aufgrund seines Menschenbilds keine klassische Gewinn-und-Verlust-Rechnung durchgeführt wird, sondern eine nach der anthroposophischen Lehre ausgerichtete Wertbildungsrechnung für materielle Werte. In dieser SEO wird daher nicht mit dem Kostenbegriff gearbeitet, sondern nur mit dem Leistungsbegriff, da hinter Kosten stets Leistungen stecken. Demzufolge gibt es z. B. auch keine klassische Werbung, sondern Public Relations, wobei ein Sog erzeugt wird über Interesse. Auf diesem Wege soll eine Ausgleichssystematik realisiert werden; Gegenwerte werden damit erkannt und sollen - im wahrsten Sinne des Wortes - Wertschätzung erfahren (IP28 ${ }^{1047}$; IP28 ${ }^{1048}$ ).

In der Gesamtbetrachtung lässt sich somit feststellen, dass argumentative Brüche im Hinblick auf den Stellenwert des Unternehmertums im Verhältnis zur sozialen Zielsetzung mit dem jeweiligen Konzeptverständnis erklärt werden können. Dabei zeigt sich bei mehreren Befragten eine konzeptionelle Unvereinbarkeit von sozialer Zielsetzung und Unternehmertum. 


\subsection{Konzeptionelle (Un-)Vereinbarkeit von sozialer Zielsetzung und Unternehmertum? Ein Typologie-Ansatz}

Wenn mehrere SE ein vergleichbares zielgerichtetes Verhalten zeigen, dann kann dies aus unterschiedlichen Beweggründen erfolgen und auf verschiedenartigen Abstraktionsniveaus verortet werden. Beispielsweise kann eine materialressourcenschonende Aktivität wie die Wiederverwertung von Versandmaterial auf einer umweltschützenden Einstellung und einem daraus resultierenden Verhalten beruhen. Dieselbe Aktivität kann ebenso z. B. auf kosteneffizientem Kalkulieren beruhen - oder aus der Kombination von Umweltbewusstsein und Wirtschaftlichkeit. Dementsprechend können mehrere Motive zu ein und demselben Zeitpunkt aktiviert und von Relevanz sein, was im Hinblick auf soziale versus wirtschaftliche Motive zu Entscheidungsdilemmata führen kann.

Wie in Abschnitt 3.4.2 skizziert, kann bereits sprachlich das Fehlen eines Konzeptansatzes, der das Soziale und das Unternehmerische angemessen verbindet, sichtbar werden. Beispielsweise wird nur die soziale Idee mit der Gestalt eines Babys attribuiert, nicht aber das Unternehmerische (IPT ${ }^{1049}$ ). In ähnlicher Weise gleicht bei IP1 soziales Handeln ab dem SEO-Gründungsprozess dem Zeugen eines Kindes: Ein Baby entsteht und es passiert organisches Wachstum, bei dem die Entwicklung des Babys erlebt und es auf dem Weg seiner reifenden Entwicklung beschützend begleitet wird (IP1: Auflistung ${ }^{1050}$ ), wobei die zwischenmenschliche Kontaktgestaltung hohe Bedeutung erfährt (IP1: Auflistung ${ }^{1051}$ ). In klarer Abgrenzung hierzu spiegelt bei IP1 das unternehmerische Gründen und betriebswirtschaftliche Handeln den Prozess des „Bauens“ wider, bei dem die Tätigkeit einem „mechanischen“ Prozess, dem Konstruieren von „Maschinen“ und von „Gebäuden“ gleicht und bei dem unvorhergesehene Baubarrieren auftreten (IP1: Auflistung ${ }^{1052}$ ).

Allerdings braucht die soziale Idee nicht nur Fürsorge, sondern auch Umsetzungsstärke (IP1 ${ }^{1053}$; im Ansatz vergleichbar diskutiert bei Miller, Grimes, McMullen \& Vogus, 2012). Die soziale Idee muss sich gegen Widerstand in der Unternehmenswelt, in der sie aufwächst, zur Wehr setzen können (IP1 ${ }^{1054}$ ). Organisches Wachstum der SEO ist auf Zuwendung angewiesen. Somit verkörpert unternehmerisches Handeln Bewegungsenergie und Antrieb (IP1: Auflistung ${ }^{1055}$ ). Insoweit basiert das sozialunternehmerische Handeln bei IP1 auf zwei verschiedenen, nicht miteinander vereinbaren Menschenbildern, die in der Folge von Widerstand und (Ab-)Brüchen geprägt sind (IP1: Auflistung ${ }^{1056}$ ): Das Baby muss im Laufe seines Lebens aus Fehlern dazulernen, aber auf eine Maschine soll von Anfang an Verlass sein. IP1 zieht dabei letztendlich den Stecker und nimmt 
ihrer Sozialunternehmung auf diese Weise die Lebensenergie. Sie setzt den Stecker weder an anderer Stelle noch auf andere Weise und ist mit ihrem Credo „Ich will es schaffen, mein Leben zu ändern!“ (IP1 $1^{1057}$ ) gescheitert; den eigenen Anspruch auf einen Transformationsprozess ,Ich schaffe es, mein Ändern zu leben!“ benennt IP1 nicht.

In der Gesamtbetrachtung wird deutlich, dass es sich bei dem Sozialen und bei dem Unternehmerischen jeweils um ein eigenständiges Phänomen handelt, wobei beide Phänomene keineswegs automatisch zueinander in Verbindung stehen (IP5 ${ }^{1058} ; \operatorname{IPp}^{1059}$, i. V. m. IPp $\left.{ }^{1060}\right)$. In diesem Sinn schildern Befragte zwei Seiten ein und derselben Medaille (IP13 ${ }^{1061}$; IP29 ${ }^{1062}$ ). Eine Befragte stellt dabei heraus, dass die inhaltliche Idee die Basis darstellt, auf der das Unternehmerische gestaltet wird. Damit kann die unternehmerische Wirksamkeit maximal so gut sein wie die inhaltliche Konzeption, bei der die inhaltliche Konzeption kontinuierlich reflektiert wird und bestmöglich optimiert bzw. an neue Umweltbedingungen und Erkenntnisse angepasst wird, also ein lebendiges, flexibles Konstrukt darstellt $\left(\mathrm{IPQ}^{1063}\right)$.

Zur Medaillen-Metaphorik mit ihren zwei Seiten passt die von Befragten ebenfalls geäußerte Zwei-Welten-Metaphorik (IP26 $6^{1064}$; IPM ${ }^{1065}$; IP1 ${ }^{1066}$ ). Dabei stellt sich die Frage, was für ein Kraftaufwand betrieben werden muss, um zwei Welten zu vereinen (IPM ${ }^{1067}$; IP $\alpha^{1068}$ ), und wer Dolmetscher für beide Welten sein kann, damit ein gegenseitiges Verständnis realisiert werden kann. Dabei wird i. d. R. weder eine Verbindungsmetaphorik noch eine Balancemetaphorik zum SE-Konzeptverständnis geäußert, sondern lediglich marginal der Anspruch des Ausbalancierens postuliert, ohne den damit verbundenen Prozess aufzuzeigen, geschweige denn, ihn kritisch zu reflektieren (IP $\varepsilon^{1069}$, i. V. m. $\left.\operatorname{IP}^{1070}\right)$.

Zudem gibt es Gründe für die verschiedenen Funktionen der zwei Welten bzw. Medaillenseiten, so dass sich die Frage stellt, weswegen bzw. wie beide Identitäten zueinander zu bringen sind. Anstatt der Vereinigung beider Welten lässt sich - in der Balancemetaphorik gesprochen - abwägen, welche Anteile der kapitalistischen Welt einen Wert für Sozialunternehmertum mitbringen, adaptierbar erscheinen bzw. anzupassen sind (IPM ${ }^{1071}$ ). Elemente aus der „kapitalistischen“ Welt werden dabei in Beziehung gesetzt zur sozialen Zielsetzung, aber nicht miteinander vereint. Bei diesem bewältigungsstrategischen Ansatz entsteht zwischen zwei Waagschalen mit ihren jeweils eigenständigen Gewichtungen ein neuer Kontakt, ohne dass die unterschiedlichen Normen bzw. Identitäten beider Welten ignoriert werden (IP23 ${ }^{1072}$ ). Social Entrepreneurship kann demnach mit der Auseinandersetzung kapitalistischer Ansätze und nicht durch deren Ablehnung gelingen $\left(\operatorname{IPr}^{1073}\right)$. 
Demzufolge ist das Ökonomische bzw. Unternehmerische nicht als Gegenpol zum Sozialen innerhalb eines Kontinuums aufzufassen, bei der weniger Ausprägung des einen Pols automatisch zur Annäherung an den anderen Pol führen würde. Verbreitet ist jedoch dieses Grundverständnis, das der Realität aber nicht gerecht wird: „Being ,purely social“ or ,purely commercial “ are considered only two poles of a continuum“ (Kruse, Wach \& Wegge, 2018, S. 5; vgl. Lepoutre, Justo, Terjesen \& Bosma, 2013, S. 709; siehe z. B. auch Majumdar \& Ganesh, 2020, S. 29; als grafische Darstellung z. B. bei Pestoff \& Hulgård, 2016, S. 1754; Rummel, 2011, S. 24; Olenga Tete, Wunsch \& Menke, 2018, S. 15; Leppert, 2013; Diochon \& Anderson, 2010, S. 101), grafisch dargestellt in Abbildung 8.6.

social $\longleftrightarrow$ entrepreneurial

Abbildung 8.6 Soziales und Unternehmerisches als zwei Dimensionen innerhalb eines Kontinuums. (z. B. nach Pestoff \& Hulgård, 2016; Rummel, 2011; Olenga Tete, Wunsch \& Menke, 2018; Leppert, 2013)

Zwar konnte ebenfalls mit Beispielen von Befragten aufgezeigt werden, dass sie dieses Entweder-oder-Prinzip verinnerlicht haben, was eine eindeutige Beurteilung mit sich bringt und somit eine inhaltliche Widersprüchlichkeit in der theoretischen Annahme ebenso ausschließt wie eine gegenseitige Beeinflussung der Dimensionen. Dieses Verständnis widerspricht gegebenenfalls einem Sowohlals-auch-Ansatz, der das Verfolgen mehrerer Zielsetzungen parallel zulässt, die eventuell erst in ihrer gemeinsamen Betrachtung unter Wahrung ihrer Eigenheiten zu weiteren, neuen Handlungen motivieren können. In diesem Sinn lässt sich mit Aussagen von Befragten aufzeigen, dass eine parallele Bilanzierung der beiden Dimensionen „Social“ und „Entrepreneurship“ erfolgt.

Vorab soll das hierbei zugrunde liegende Phänomen illustriert werden am Beispiel der asiatischen kulinarischen Köstlichkeit einer Speiseeiskugel, die im gefrorenen Zustand in einem heißen frittierten Teigmantel serviert wird und die erst in dieser Komposition ein ganz eigenes Geschmackserlebnis ermöglicht. Werden die verschiedenen Bestandteile in Verbindung zueinander wahrgenommen, also zeitgleich, dann entsteht ein eigener Erfahrungswert, der in seiner Heiß-kaltKomposition als widersprüchlich bewertet werden kann. Das Neue - in diesem Fall das subjektiv als einzigartig empfundene Geschmackserlebnis - und der dabei ebenso subjektiv vorgenommene Bewertungsprozess entstehen somit erst durch die Gleichzeitigkeit, in der die verschiedenen Elemente wirken, also durch 
Parallelität. Bei diesem Verständnis besteht die Gesamtstruktur aus zwei eigenständigen Teilen, die keine Schnittmenge besitzen, sondern als komplementäres Gebilde eine übergeordnete Einheit im Sinne einer Dichotomie bilden.

Dieses Phänomen - also das Vornehmen von Bewertungen bei parallel ablaufenden Prozessen, die subjektiv empfunden nicht zueinander passen - kann ebenfalls bei der (Selbst-)Bewertung des sozialunternehmerischen Denkens und Handelns zutage treten. Denn eine innere Widersprüchlichkeit in der Beziehung zwischen „Social“ und „Entrepreneur“ lässt sich nicht ausschließen, so dass ein Beurteilungsergebnis i. d. R. weder einfach noch eindeutig abfragbar ist.

$\mathrm{Zu}$ Beginn von Kapitel 8 wurde thematisiert, dass die Bedürfnisse, Motive und Einstellungen einer Person durchaus miteinander konfligieren. Dabei wirken sie als Bestandteile eines Bedingungsgefüges; je nach Situationskontext können unterschiedliche Verhaltensweisen gezeigt werden. Um den Handlungsmotivationsprozess von SE mit seinen Eigenarten verstehen zu können, ist somit weder der Durchschnittswert zu Bedürfnissen, Motiven und Einstellungen heranzuziehen noch der Durchschnittswert zu gezeigten Verhaltensweisen. Durchschnittswerte können nicht die verschiedenen Qualitäten des Denkens und Handelns repräsentieren. An welchen Stellen sich innere Widersprüche auf das eigene sozialunternehmerische Denken und Handeln auswirken und inwieweit hierbei Widersprüche bestehen, die für SE typisch sind und die sich speziell auf deren Belastungserleben auswirken können, ist im Folgenden herauszuarbeiten.

Unter Hinzuziehen der motivationspsychologischen Ausführungen, insbesondere zum Stellenwert von Bedürfnissen und zur Zielsetzung, zeigt sich ein sogar noch komplexerer Zuordnungsprozess mit einer zu bilanzierenden Bewertungsdynamik. Denn in der Zusammenschau treten nicht nur das Soziale und das Unternehmerische als eigenständige Kriterien i. S. v. Dimensionen auf. Darüber hinaus können innerhalb ein und derselben Dimension Widersprüche auftreten, für die abermals ein Verbindungskonzept fehlt. Grundsätzlich bestehen hierbei vier gleichwertig anzusehende, nicht in direkter Verbindung stehende Kriterien (grafisch wiedergegeben in Abbildung 8.7):

- eine positive Einstellung gegenüber dem Sozialen/ein soziales Denken und Handeln (abgekürzt: ,sozial positiv“),

- eine negative Einstellung gegenüber dem Sozialen/ein „unsoziales“ Denken und Handeln (abgekürzt: „,sozial negativ“),

- eine positive Einstellung gegenüber dem Unternehmerischen/ein Denken und Handeln nach unternehmerischen Grundsätzen (abgekürzt: ,unternehmerisch positiv") und 
- eine negative Einstellung gegenüber dem Unternehmerischen/ein Denken und Handeln, das sich den unternehmerischen Grundsätzen widersetzt (abgekürzt: „unternehmerisch negativ“).

\author{
Abbildung 8.7 Soziales \\ und Unternehmerisches als \\ vier gleichwertig \\ anzusehende, nicht in \\ direkter Verbindung \\ stehende Kriterien. (eigene \\ Darstellung)
}
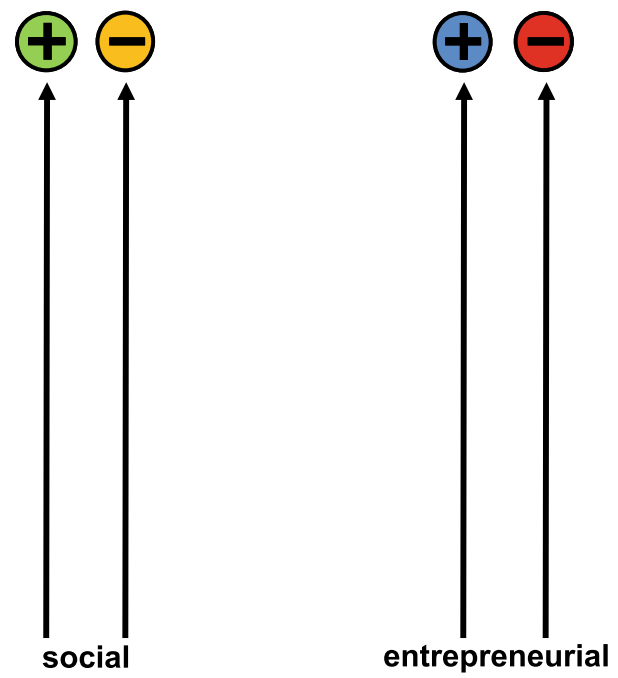

Zwar ließe sich als Antagonist zu „sozial“ der Begriff „unsozial“ heranziehen - weitere Wortkombinationen mit Präfixen wie „dis-“ oder ,anti-“ erweisen sich als ungeeignet, da sie im Rahmen der Psychopathologie beispielsweise zur Bestimmung von Persönlichkeitsstörungen genutzt werden. Zur Wahrung der Vergleichbarkeit aller vier Kriterienbezeichnungen wird jedoch auf die Verwendung von ,unsozial“" verzichtet. Denn als lexikalisch-semantische Schwierigkeit erweist sich, dass es in der deutschen Sprache für das Gegenteil von unternehmerisch keinen geeigneten Begriff gibt. „Ununternehmerisch“ erscheint in der Abgrenzung nicht eindeutig, so dass hierauf verzichtet wird.

Unter ,sozial positiv“ wird das vom Individuum konstruktiv gestaltete und dem Gemeinwohl dienliche zwischenmenschliche Kommunizieren und Kooperieren verstanden, geprägt von Förderung der Gruppenzugehörigkeit aller Mitglieder und einem wertschätzenden, respektvollen Umgang miteinander, wobei eine eigene Vorteilnahme zum Nachteil Dritter bewusst vermieden wird. Aus 
den Beziehungen, die Individuen in ihren gesellschaftlichen Gruppen zueinander haben, werden Kontakte gestaltet, die beispielsweise eine Vertrauensbasis schaffen können.

Demzufolge wird unter ,sozial negativ“ eine von Widerstand gekennzeichnete Beziehungsgestaltung verstanden, die instabile Beziehungsstrukturen festigt sowie eine Misstrauenskultur fördert und in der sich abwertend, despektierlich gegenüber Dritten geäußert wird. Zudem erweist sich ein Denken und Handeln als sozial negativ, wenn es sich gegen die Interessen sozial Schwächerer richtet; eine eigene Vorteilnahme zum Nachteil Dritter kann beabsichtigt sein.

Unter ,unternehmerisch positiv“ wird das zielsetzungsverankerte Planen und Umsetzen von Entscheidungen und Maßnahmen verstanden, wobei der Handlungsspielraum unter Berücksichtigung von Aus-, Neben- und Wechselwirkungen sowohl innerhalb des agierenden Systems als auch im Hinblick auf externe Partner(-organisationen) kontinuierlich und prozessorientiert kalkuliert, strategisch genutzt und kompetenzbasiert ausgeschöpft wird; dabei werden die Aktivitäten ebenso im Hinblick auf den Bedeutungsgehalt des eigenen Systemerhalts reflektiert.

„Unternehmerisch negativ“ ist gekennzeichnet von Widerstand gegenüber dem Gestalten und Befolgen erreichbarer und realisierbarer Zielsetzungen. Dem eigenen Handlungsspielraum wird sich widersetzt und es wird aversiv gegenüber dem Treffen von Entscheidungen aufgetreten bzw. werden Entscheidungen konzeptlos, kurzsichtig oder engstirnig getroffen. Aus-, Neben- und Wechselwirkungen sowohl innerhalb des agierenden Systems als auch im Hinblick auf externe Partner(-organisationen) werden somit ignoriert bzw. missachtet und Handlungsabläufe isoliert voneinander betrachtet. Die Gefährdung des Systemerhalts kann hierbei provoziert werden.

$\mathrm{Zu}$ erkennen sind vier eigenständige, nicht in direkter Verbindung stehende Kriterien i. S. v. Dimensionen. Schematisch wird an dieser Stelle unterschieden zwischen:

- schwach ausgeprägt,

- mäßig ausgeprägt und

- stark ausgeprägt.

Demnach kann jede der vier Dimensionen von schwach über mäßig bis stark ausgeprägt sein. Beispielsweise stellt eine nicht stark positiv ausgeprägte soziale Positionierung keineswegs per se eine negative Ausprägung dar, sondern nur eine mäßig bzw. schwach positive Ausprägung. Diese Differenzierung erweist sich im Hinblick auf die zentrale Forschungsfrage als sehr bedeutsam: 
Im Anhang L im elektronischen Zusatzmaterial zeigen drei Abbildungen hierzu exemplarisch für drei IP unterschiedliche Positionierungen gegenüber „,social“ und ,entrepreneurial“, also in unterschiedlicher Intensität und auszugsweise mit typischen Aussagen. Die erste der drei Abbildungen stellt dies für IP1 dar. In einem zweiten Beispiel mit Zitaten von IP2 positioniert sich dieser IP gegenüber „social“ ausschließlich positiv, dies sogar extrem stark ausgeprägt. Darüber hinaus wird mit dem dritten Beispiel zu IP33 ein SE aufgezeigt, der in allen vier Kriterien verhältnismäßig stark ausgeprägte Positionierungen vorweist (in allen drei Beispielen auszugsweise mit Zitaten dargestellt). Die dargestellten IP-Beispiele mit deren Verortung innerhalb der vier Dimensionen verdeutlichen Inkonsistenzen in Bewertungsvorgängen: sowohl innerhalb „social“ als auch innerhalb „entrepreneurial“ als auch im Verhältnis zwischen „,social“" und „entrepreneurial“.

Abbildung 8.8 zeigt für die im Anhang L (elektronisches Zusatzmaterial) wiedergegebene Positiv-Negativ-Bilanzierung der drei IP eine andere Darstellungsform, die es ermöglicht, den Sachverhalt mit SE-spezifischen Profilen im Spinnendiagramm zu erfassen.

Da in der vorliegenden Untersuchung aus arbeits- und organisationspsychologischer Perspektive Unterschiede sowohl zwischen SE als auch in Abgrenzung zu KE herausgearbeitet werden, erscheint es im Sinne des damit gegebenen Erkenntnisinteresses zweckmäßig, eine hierfür geeignete Fokussierung vorzunehmen. In diesem Sinn wird eine definitorische Differenzierung zugrunde gelegt, demgemäß SE, die eine schwache Ausprägung im sozial Positiven und eine starke Ausprägung im sozial Negativen mitbringen, von der Zugehörigkeit zum sozialunternehmerischen Soll-Profil ausgeschlossen werden. Hypothetisch wird angenommen, dass SE als Basis immer eine mindestens mittelmäßig ausgeprägte positive soziale Einstellung bzw. ein mindestens mittelmäßig ausgeprägtes positives soziales Denken und Handeln mitbringen; ebenso zeigen sie eine höchstens mittelmäßig ausgeprägte negative soziale Einstellung bzw. ein höchstens mittelmäßig ausgeprägtes negatives soziales Denken und Handeln. Wohlgemerkt passiert diese Differenzierung der zuvor benannten Zweckmäßigkeit halber, also bei dem gegebenen Erkenntnisinteresse als kontingente Entscheidung. Somit wird keinesfalls beurteilt, ob ein IP ein Sozialunternehmer ist oder nicht, sondern nur, wo er im Rahmen des Forschungsprozesses bezogen auf die vorgenommene Operationalisierung verortet werden kann.

In der Gesamtbetrachtung lassen sich bei diesem Verständnis SE im Rahmen einer Typologie zuordnen mit 36 Kombinationsmöglichkeiten. Auf Basis der motivationspsychologischen Ausführungen - wenngleich unter der empirisch 


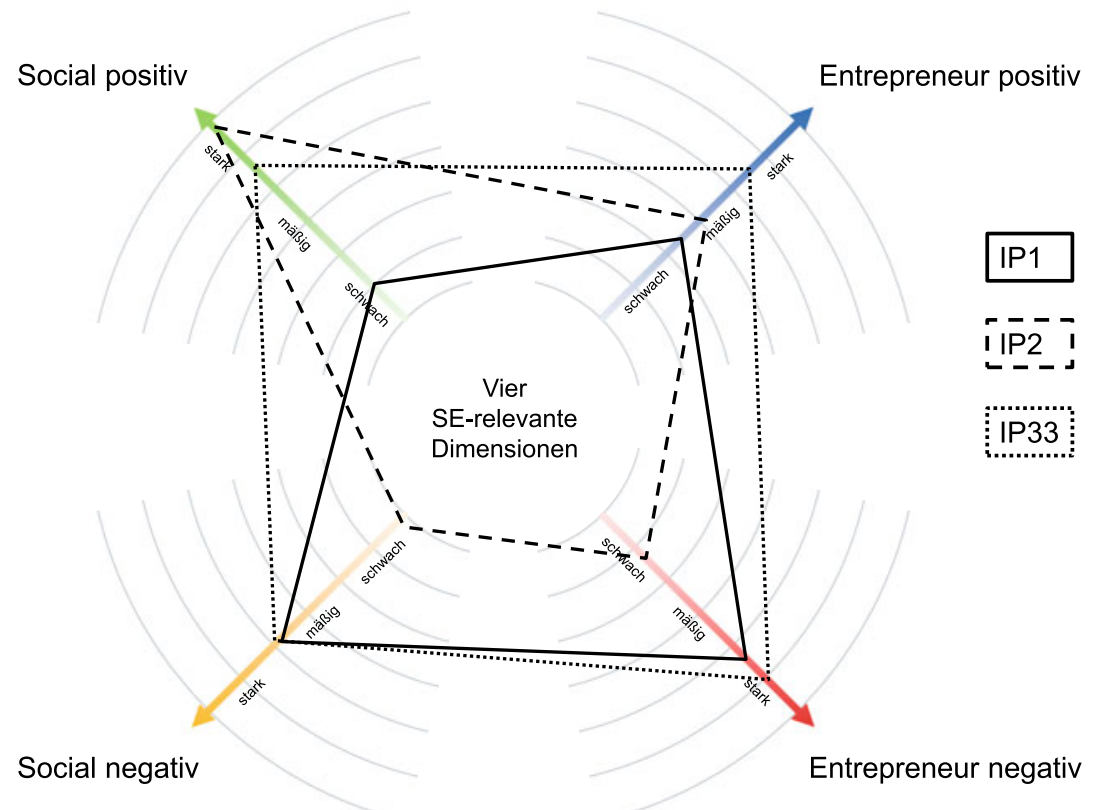

Abbildung 8.8 Spinnendiagramm mit IP-Beispielprofilen zu vier gleichwertig anzusehenden Kriterien für Soziales und Unternehmerisches. (eigene Darstellung)

nicht belegten Annahme und insofern ebenfalls der oben benannten ZweckmäBigkeit halber und bei dem gegebenen Erkenntnisinteresse als kontingente Entscheidung getroffen - wird davon ausgegangen, dass bei SE, die bereits eine SEO gegründet haben, die Handlungsmotivationskraft ihrer Hin-zu-Ziele stärker ist als die Handlungsdemotivationskraft ihrer Zustände, von denen sie sich distanzieren wollen. Dies erlaubt, positive Einstellungen und das positive Denken/Handeln prominent zu betrachten. Demgemäß lassen sich die negativen Aspekte den positiven Aspekten in ihrer Relevanz unterordnen. Bei diesem Denkansatz können sechs prominente SE-Typen identifiziert werden, die in jeweils sechs Untertypen ausgeprägt sein können und die sich demzufolge in ihren positiven Einstellungsmustern gleichen, sich aber in der Ausprägung ihrer negativen Einstellungsmuster unterscheiden. Tabelle 8.1 spiegelt diesen Differenzierungsansatz wider; dabei treten 36 SE-Grundtypen in Erscheinung. 
Tabelle 8.1 36 SE-Grundtypen. (eigene Darstellung)

\begin{tabular}{|c|c|c|c|c|}
\hline \multirow{2}{*}{ Typ-Nr. } & \multicolumn{2}{|c|}{ Prominenter Typ } & \multicolumn{2}{|c|}{ Untertyp } \\
\hline & Social: positiv & $\begin{array}{c}\text { Entrepreneurship: } \\
\text { positiv }\end{array}$ & Social: negativ & $\begin{array}{c}\text { Entrepreneurship: } \\
\text { negativ }\end{array}$ \\
\hline 1 & \multirow{6}{*}{$\begin{array}{c}\text { stark } \\
\text { ausgeprägt }\end{array}$} & \multirow{6}{*}{$\begin{array}{c}\text { stark } \\
\text { ausgeprägt }\end{array}$} & schwach ausgeprägt & schwach ausgeprägt \\
\hline 2 & & & schwach ausgeprägt & mäßig ausgeprägt \\
\hline 3 & & & mäßig ausgeprägt & schwach ausgeprägt \\
\hline 4 & & & mäßig ausgeprägt & mäßig ausgeprägt \\
\hline 5 & & & mäßig ausgeprägt & stark ausgeprägt \\
\hline 6 & & & schwach ausgeprägt & stark ausgeprägt \\
\hline 7 & \multirow{6}{*}{$\begin{array}{c}\text { stark } \\
\text { ausgeprägt }\end{array}$} & \multirow{6}{*}{$\begin{array}{c}\text { mäßig } \\
\text { ausgeprägt }\end{array}$} & schwach ausgeprägt & schwach ausgeprägt \\
\hline 8 & & & schwach ausgeprägt & mäßig ausgeprägt \\
\hline 9 & & & mäßig ausgeprägt & schwach ausgeprägt \\
\hline 10 & & & mäßig ausgeprägt & mäßig ausgeprägt \\
\hline 11 & & & schwach ausgeprägt & stark ausgeprägt \\
\hline 12 & & & mäßig ausgeprägt & stark ausgeprägt \\
\hline 13 & \multirow{6}{*}{$\begin{array}{c}\text { stark } \\
\text { ausgeprägt }\end{array}$} & \multirow{6}{*}{$\begin{array}{l}\text { schwach } \\
\text { ausgeprägt }\end{array}$} & schwach ausgeprägt & schwach ausgeprägt \\
\hline 14 & & & schwach ausgeprägt & mäßig ausgeprägt \\
\hline 15 & & & schwach ausgeprägt & stark ausgeprägt \\
\hline 16 & & & mäßig ausgeprägt & schwach ausgeprägt \\
\hline 17 & & & mäßig ausgeprägt & mäßig ausgeprägt \\
\hline 18 & & & mäßig ausgeprägt & stark ausgeprägt \\
\hline 19 & \multirow{6}{*}{$\begin{array}{c}\text { mäßig } \\
\text { ausgeprägt }\end{array}$} & \multirow{6}{*}{$\begin{array}{c}\text { stark } \\
\text { ausgeprägt }\end{array}$} & schwach ausgeprägt & schwach ausgeprägt \\
\hline 20 & & & schwach ausgeprägt & mäßig ausgeprägt \\
\hline 21 & & & schwach ausgeprägt & stark ausgeprägt \\
\hline 22 & & & mäßig ausgeprägt & schwach ausgeprägt \\
\hline 23 & & & mäßig ausgeprägt & mäßig ausgeprägt \\
\hline 24 & & & mäßig ausgeprägt & stark ausgeprägt \\
\hline 25 & \multirow{6}{*}{$\begin{array}{c}\text { mäßig } \\
\text { ausgeprägt }\end{array}$} & \multirow{6}{*}{$\begin{array}{c}\text { mäßig } \\
\text { ausgeprägt }\end{array}$} & schwach ausgeprägt & schwach ausgeprägt \\
\hline 26 & & & schwach ausgeprägt & mäßig ausgeprägt \\
\hline 27 & & & schwach ausgeprägt & stark ausgeprägt \\
\hline 28 & & & mäßig ausgeprägt & schwach ausgeprägt \\
\hline 29 & & & mäßig ausgeprägt & mäßig ausgeprägt \\
\hline 30 & & & mäßig ausgeprägt & stark ausgeprägt \\
\hline 31 & \multirow{6}{*}{$\begin{array}{c}\text { mäßig } \\
\text { ausgeprägt }\end{array}$} & \multirow{6}{*}{$\begin{array}{l}\text { schwach } \\
\text { ausgeprägt }\end{array}$} & schwach ausgeprägt & schwach ausgeprägt \\
\hline 32 & & & schwach ausgeprägt & mäßig ausgeprägt \\
\hline 33 & & & schwach ausgeprägt & stark ausgeprägt \\
\hline 34 & & & mäßig ausgeprägt & schwach ausgeprägt \\
\hline 35 & & & mäßig ausgeprägt & mäßig ausgeprägt \\
\hline 36 & & & mäßig ausgeprägt & stark ausgeprägt \\
\hline
\end{tabular}


Abbildung 8.9 fängt die ergänzende Ausführung zur Typologie auf und zeigt die in Abbildung 8.8 bereits besprochenen Positionierungen für IP1, IP2 und IP33 in modifizierter Form. Durch den Ausschluss der schwachen Ausprägung im sozial Positiven und der starken Ausprägung im sozial Negativen fällt nun ins Gewicht, dass IP1 eine auffallend schwach ausgeprägte positive soziale Positionierung verkörpert, zudem eine relativ stark ausgeprägte negative soziale Positionierung und mit ihrem Profil somit nicht mehr innerhalb der vorgegebenen SE-Kategorienausprägungen liegt.

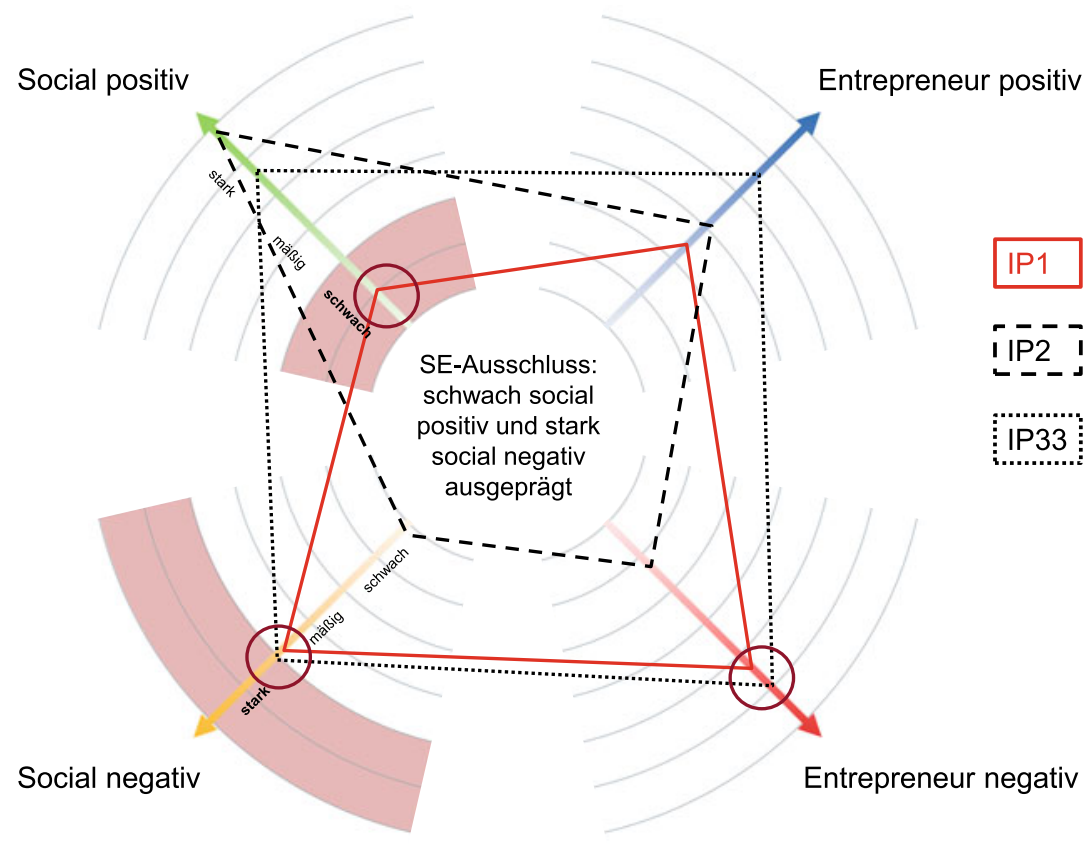

Abbildung 8.9 Modifizierte Vorlage zum Spinnendiagramm mit IP-Beispiel-Profilen zu vier gleichwertig anzusehenden Kriterien für Soziales und Unternehmerisches, ergänzt um ein Soll-Profil. (eigene Darstellung)

Bei der Reflexion, was dies für das Verorten von IP1 in der Gesamtgruppe der SE bedeuten kann, ist einzubeziehen, dass die stark untergeordnete Rolle des Sozialen im „Sozialunternehmertum“ von IP1 eine deutliche Abgrenzung 
gegenüber allen anderen Befragten aufzeigt. Die Tatsache, vielfach als SE ausgezeichnet worden zu sein, sollte nicht über diesen Missstand hinwegtäuschen; dies wurde bereits diskutiert. Damit wird an dieser Stelle untermauert, dass aus einer lebenslangen Auszeichnung als SE keinesfalls eine dauerhaft gegebene Handlungsmotivation abgeleitet werden kann, geschweige denn das Vollziehen von Handlungen. Keineswegs wird dabei für IP1 in Abrede gestellt, dass im Kontext der beschriebenen beruflichen Auszeit mit Urlaubsatmosphäre fernab vom Heimatland der Ursprung für die Intentionsbildung zum sozialunternehmerischen Gründungsvorhaben zu finden ist (IP1 ${ }^{1074}$ ) und dass es in der Folge zur Gründung gekommen war mit sozialunternehmerischen Absichten. Allerdings sprechen für den weiteren Verlauf zahlreiche Widersprüche in den inhaltlichen Aussagen eine eigene Sprache; sie spiegeln ein in der Vergangenheit liegendes temporäres Interesse für Sozialunternehmertum wider. Zudem entsprechen diverse Äußerungen dieser Befragten dem oben beschriebenen Verständnis von „sozial negativ“, was bei einer Gesamtbewertung wie dargestellt einzubeziehen ist und die Abgrenzungsthese zusätzlich stützt. Insofern stellt sich eine weitere grundsätzliche Frage, nämlich inwieweit für die in Kapitel 2 dargestellten Untersuchungserkenntnisse zur Social-Entrepreneurship-Intention an Aussagekraft verlieren können im Hinblick auf SE, die bereits gegründet haben.

Die vier sozialunternehmerischen Kriterien können sich in ihren Ausprägungen also verändern, sie stellen flexible Strukturen dar. Erinnert wird als Beispiel für eine positive Entwicklung an den in Abschnitt 5.3 zu IP26 dargestellten Lernprozess, bei dem eine soziale Idee in seiner Gedankenwelt heranreifte, es ihm jedoch an unternehmerischer Handlungskompetenz mangelte. Erst nachdem er das betriebswirtschaftliche Know-how erworben hatte, kam sein unternehmerisches Handeln zum Einsatz (IP26 ${ }^{1075}$ ).

In vergleichbarer Weise können aus sozialunternehmerischer Sicht negative Entwicklungen eintreten. In diesem Sinn sollte z. B. die Selbstoffenbarung "Ich bin Sozialunternehmer" relativiert werden und - wie für IP1 aufgezeigt selbstkritisch als Rückblick erfolgen, in etwa: „Ich hatte in der Vergangenheit sozialunternehmerische Ambitionen, war aber nur für kurze Zeit als Sozialunternehmer aktiv“. Dies kann der Transparenz dienlich sein. Darüber hinaus lässt sich eine noch differenziertere Betrachtung ableiten. Denn ein Entrepreneur, der im Besitz mehrerer Unternehmen ist, kann dabei ggf. herausstellen: „Mit meinem Geschäftsmodell A agiere ich im Marktgeschehen sozialunternehmerisch, aber mit meinem Geschäftsmodell B trete ich nicht als Sozialunternehmer auf, sondern betreibe ein konventionelles Unternehmen “. Dies trifft beispielsweise zu auf IPS 
(IPS ${ }^{1076}$ ). Ebenso kann IPY, die zusätzlich im Rahmen eines Beschäftigungsverhältnisses eine KEO in deren Corporate-Social-Responsibility-Aktivitäten unterstützt (IPY ${ }^{1077}$ ), dies deutlich von ihrem sozialunternehmerischen Engagement abgrenzen. Derartige Differenzierungen könnten einer Instrumentalisierung von lebenslangen SE-Auszeichnungen entgegenwirken und die Gefahr von Fehlkonnotationen in der Öffentlichkeit vermeiden.

Allerdings ist in logischer Konsequenz eine weitere denkbare Einschränkung bei dem getroffenen Typologie-Ansatz zu reflektieren. Schließlich wurde eingangs unterstellt, dass eine stark positive soziale Einstellung in Kombination mit einer stark positiven unternehmerischen Einstellung eine sozialunternehmerisch günstige Position darstellt. Insofern kann vergleichbar zum Sozialen für das Unternehmerische angenommen werden, dass eine schwach positive Ausprägung des Unternehmerischen der Zugehörigkeit zum Sozialunternehmertum bei diesem Typologie-Ansatz widerspricht. Denn ohne positiv ausgeprägtes unternehmerisches Basisverständnis kann es am zielsetzungsverankerten Planen und Umsetzen von Entscheidungen und Maßnahmen mangeln: Denkbar ist beispielsweise, dass der Handlungsspielraum nicht hinreichend unter Berücksichtigung von Aus-, Neben- und Wechselwirkungen sowohl innerhalb der Organisation als auch im Hinblick auf externe Partner kontinuierlich und prozessorientiert kalkuliert, strategisch genutzt und kompetenzbasiert ausgeschöpft wird; ggf. werden Aktivitäten nicht in dem erforderlichen Ausmaß bezogen auf den Bedeutungsgehalt des eigenen Systemerhalts reflektiert. Demzufolge schließt sich aus, dass SE eine schwach ausgeprägt positive Einstellung gegenüber dem Unternehmerischen und gegenüber dem Denken und Handeln nach unternehmerischen Grundsätzen verkörpern.

Jedoch erscheint hierbei infolge der Datenauswertung eine Differenzierung erforderlich: Aufgezeigt wurde, dass mehrere SE ihre Defizite im Hinblick auf ihr unternehmerisches Kompetenzprofil deutlich benennen können und demzufolge in Teamstrukturen zusammenarbeiten (wollen), so dass die bestehenden Missstände nicht nur kompensiert, sondern präventiv und strategisch bewältigt werden (sollen). Einerseits zeigen diese IP damit eine positive Einstellung gegenüber dem Unternehmerischen, wenngleich diese positive Einstellung in der Gesamtbetrachtung als mäßig ausgeprägt zu bewerten ist. Andererseits denken und vor allem handeln sie nicht in ausgeprägter Form unternehmerisch, obwohl deren Teamarbeit-Bewältigungsstrategie im Ansatz unternehmerisches Denken widerspiegeln mag. Daher ist ihre Positionierung gegenüber dem Unternehmerischen als schwach positiv zu bewerten. Gleichwohl kann das hier geschilderte Hinzuziehen geeigneter Partner in seiner Umsetzung von unternehmerischem Erfolg gekrönt sein $\left(\right.$ IP6 $\left.^{1078}\right)$. 
Darüber hinaus ist differenziert zu betrachten, welche Auswirkungen eine stark negative Ausprägung des Unternehmerischen haben kann. Beispielsweise erscheint es denkbar, dass ein SE seine Bedenken und Zweifel auf Basis einer stark positiv ausgeprägten sozialen und unternehmerischen Positionierung kritisch reflektiert und sie letztendlich relativiert. Eventuell bekundet ein entsprechender SE von sich aus Klärungsbedarf in seinem Entscheidungsmanagement oder ist offen gegenüber Hilfestellungen durch SE-Berater.

Aus diesen Überlegungen heraus sollen im Typologie-Ansatz eine schwach ausgeprägt positive unternehmerische Positionierung sowie eine stark ausgeprägt negative unternehmerische Positionierung weiterhin Berücksichtigung finden (Abbildung 8.10), allerdings mit den beschriebenen Einschränkungen bei der Charakterisierung dieser Typen und insofern in Habachtstellung gesetzt.

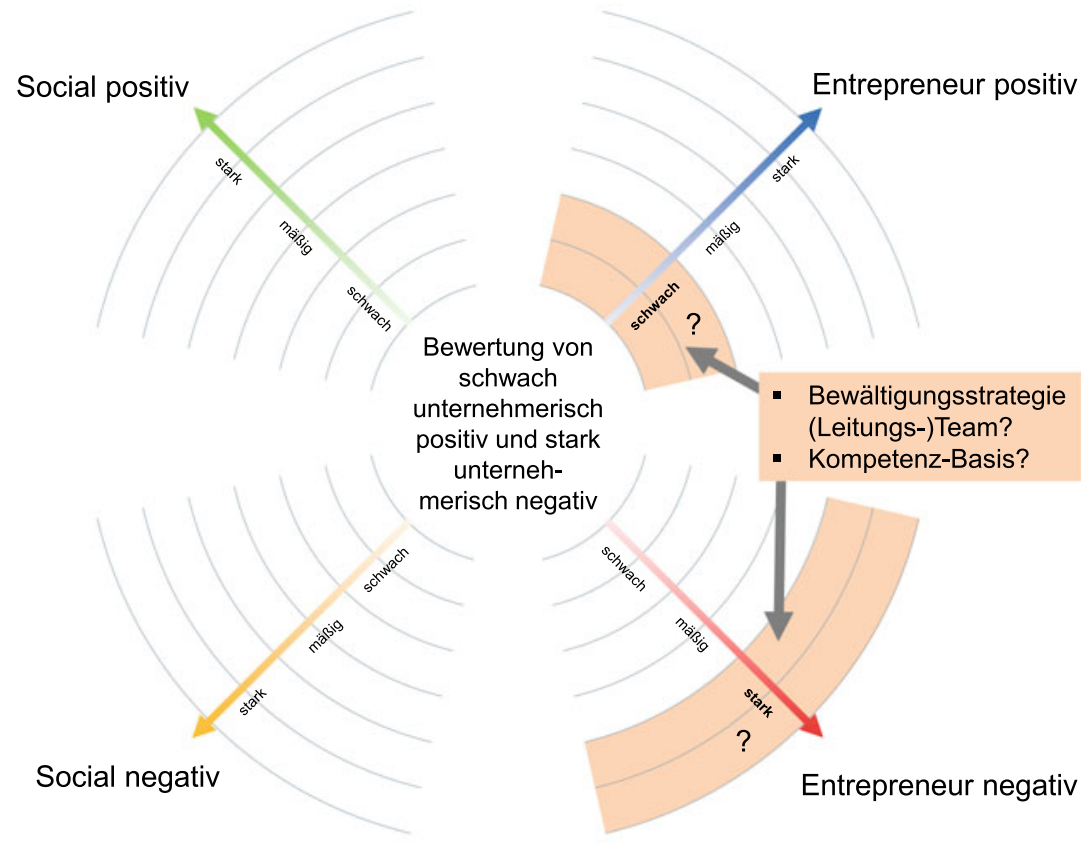

Abbildung 8.10 Spinnendiagramm-Vorlage zu vier gleichwertig anzusehenden Kriterien für Soziales und Unternehmerisches, mit Hinweisen zur Einschränkung unternehmerischer Ausprägungen. (eigene Darstellung) 
Wie dargestellt, bestehen die Definitionen zu „sozial positiv“, „sozial negativ“, „unternehmerisch positiv“ und „unternehmerisch negativ“ jeweils aus mehreren Elementen. Dabei kann jeder Definitionsbestandteil bei jedem IP in unterschiedlicher Ausprägung vorliegen. Demzufolge kann die Kennzeichnung auf der Skala nur einen Durchschnittswert für die Zusammenschau aller Definitionsbestandteile darstellen. Zudem kann jeder einzelne Definitionsbestandteil inkonsistent gelebt werden. Beispielsweise kann sich ein und dieselbe Person in einer Situation stark sozial positiv verhalten und in anderen Situationen schwach sozial positiv, so dass die Kennzeichnung auf der Skala ebenfalls nur einen Durchschnittswert abbilden kann. Es wurde bereits dargelegt, dass Durchschnittswerte hierbei nicht aussagekräftig sein können, wohl aber die dahinterliegenden personenbezogenen Werte. Widersprüchlichkeiten lassen sich durch die Differenzierung zwischen dem Negativ- und dem Positivkriterium ansatzweise herausarbeiten.

Augenscheinlich kann bei der geschilderten Differenzierung der Eindruck erweckt werden, dass qualitative Daten quantifiziert wurden und die herausgearbeiteten angeordneten Ergebnisse wieder in qualitative Kategorien rückgeführt wurden. Dies ist zu relativieren. Denn es versteht sich von selbst und wird dennoch ausdrücklich betont, dass bei diesem Vorgehen eine quantitativ abgesicherte Skalierung von IP-Daten weder gewährleistet werden kann noch beabsichtigt ist, da die Zuordnung der Aussagen einer IP nicht auf Basis operationalisierter Skalen und statistischer Güte zu erfolgen hat, sondern ein Interpretationsspielraum besteht. Vielmehr lässt sich von sämtlichen Einzelfällen ausgehend ein identischer Abstrahierungsprozess darstellen, und auf dieser Basis lassen sich die aufgezeigten Typen bilden. Dass bei dieser beabsichtigten In-etwa-Zuordnung weder ein Anspruch auf Treffgenauigkeit noch auf Eindeutigkeit bestehen kann, belegen bereits die hierzu im Rahmen von Kodier-Arbeitsgruppen vollzogenen Interviewanalysen, wenn komparativ eine Verortung eines IP diskutiert wurde. Diese Analysen belegen jedoch in gleicher Weise die intersubjektiv nachvollziehbare Zuordnung auf einem Spektrum für alle vier Kriterien, und genau hierin besteht die Güte des Vorgehens: Es gibt für alle SE Ausprägungen auf allen vier Skalen, so dass sich vom Einzelfall abstrahierend eine idealisierte Typenbildung vornehmen lässt und sich somit der Typologie-Ansatz als schlüssig und erkenntniserweiternd erweist.

\section{Implikation des Typologie-Ansatzes für die Praxis und kritische Reflexion}

Dabei zeigt es sich für die Verwendung des dargestellten Typologie-Ansatzes als vorteilhaft, dass er für verschiedene Fragestellungen zur Situationsanalyse herangezogen werden kann: 
Beispielsweise wurde in den bisherigen Diskussionskapiteln herausgearbeitet, dass Merkmale wie z. B. Infrastrukturen zu SEO-U und zur Gesetzgebung belastend auf SE wirken können und dass die von diesen Infrastrukturen ausgehenden Beziehungsgestaltungen eigene Bedingungen i. S. v. Dynamiken mit sich bringen können, die auf den SE belastend einwirken. In vergleichbarer Weise lässt sich ein Typus aus dem beschriebenen Typologie-Ansatz als Merkmal eines SE begreifen, das wiederum im sozialen Beziehungsgefüge sowie in intrapsychischen Konfliktsituationen dynamisch belastend wirkt. Diese spezifischen Belastungssituationen führen wiederum zur Beanspruchung und können eine besondere Unterstützung für SE einfordern, damit sie mentale wie soziale Konflikte bewältigen können. Für das Herausarbeiten der dabei gegebenen Widersprüchlichkeiten in Konfliktsituationen kann der Typologie-Ansatz zur Konzeption eines methodischen Vorgehens im Praxisfeld, z. B. von SEO-Beratern, herangezogen werden. Dabei kann Unterstützungsbedarf typbezogen präzisiert werden.

Darüber hinaus kann der Typologie-Ansatz herangezogen werden zum Selbstbild-Fremdbild-Abgleich oder zur Klärung moralischer Dilemmata, im Rahmen einer Leitbildentwicklung mit ihrer Missions-Visions-Verankerung bis zur Zuordnung bei Wirkungsmessungen, wenn z. B. eine Verbindung zum Unternehmerischen zu verorten ist (IP $\left.\varepsilon^{1079}\right)$. Entwicklungsziele können formuliert und grafisch zugeordnet werden.

Ebenso lassen sich die verschiedenen Definitionsansätze zum Social Entrepreneurship neu verorten. Dabei erscheint ein erweitertes Verständnis für die bisherigen Erkenntnisse ebenso denkbar; beispielsweise im Hinblick auf Intentionsbildung, wenn eine Zuordnungsmöglichkeit nicht nur im Hinblick auf Einstellungen besteht, sondern im Abgleich zu durchgeführten Handlungen gespiegelt und bewertet werden kann. Zudem lassen sich intrapersonale Vergleiche - z. B. Veränderungen der Einstellungs- und Handlungsmuster eines SE im zeitlichen Verlauf - ebenso darstellen wie interpersonale Vergleiche zwischen Einstellungen und Handlungen mehrerer SE, etwa von Co-Foundern.

In jedem Fall ist zu berücksichtigen, dass eine maximal denkbare Ausprägung einer Dimension nicht per se anzustreben ist. Vielmehr sind Zuordnungen auf Basis von Soll- und Ist-Profilen vorzunehmen. Beispielsweise erscheint es denkbar, dass eine maximal positive Ausprägung des Sozialen zu Verblendungseffekten für das Unternehmerische führen kann, wenngleich sie keineswegs zwangsläufig dazu führen muss. Die Ausführung in Abschnitt 8.11 zur Gutgläubigkeit eines Befragten (IPJ) belegt, dass dies vorkommen kann. In diesem Sinn lässt sich die Notwendigkeit von Soll-Profilen indirekt ebenso aus internationalen Studien zur SE-Typologie ableiten (vgl. insbesondere Abebe, Kimakwa \& Redd, 2020), wenngleich Klassifizierungen zu nicht nachvollziehbaren Ausgrenzungen 
führen (ebd.), die dargestellten Typologie-Ansätze insgesamt sehr unspezifisch, teilweise inkonsistent sind oder bei unzureichender empirischer Datenlage eher Hypothesen formulieren bzw. sich auf die Social-Entrepreneurship-Intention beziehen (vgl. Abschn. 2.1.5; Abschn. 2.2; Miculaiciuc, 2019; Tुigu, Iorgulescu, Răvar \& Lile, 2015; Jarrodi, Byrne \& Bureau, 2019; zur Übersicht zu SEOTypologie-Ansätzen siehe Erpf, Tekula \& Neuenschwander, 2019). Daher werden sie an dieser Stelle nicht wieder aufgegriffen bzw. vertieft (zur Limitation der Persönlichkeitstypologie von SE siehe Abschn. 8.13).

Bei der angenommenen Inferiorität von negativen Positionierungen kann der Eindruck erweckt werden, dass in Entscheidungssituationen im sozialunternehmerischen Alltagsgeschehen eine mäßig soziale positive Ausprägung prominenter erscheint und bedeutsamer wirkt als eine mäßig soziale negative Ausprägung. Allerdings sind derartige Rückschlüsse nicht einfach ableitbar. Sicherlich sollte berücksichtigt werden, dass eine stark negativ ausgeprägte soziale Positionierung bei einer nur mäßig positiv ausgeprägten sozialen Positionierung verhältnismäBig stärker ins Gewicht fallen sollte, als wenn die sozial positive Einstellung ebenfalls stark ausgeprägt ist. Denn bei dominierender Demotivation kann die unterliegende Antriebsstärke zum Erliegen kommen (vgl. z. B. Roth \& Dicke, 2005; LeDoux, 2000; Storch \& Krause, 2014). Für SE, die z. B. mit stark eskalierten Teamkonflikten inklusive persönlicher Kränkungen zu kämpfen haben, kann dies dementsprechend zur Kapitulation führen. Jedoch sind viele weitere Einflusskriterien zu berücksichtigen, beispielsweise die in Abschnitt 8.7 im Rahmen des Anspruchsniveaus zur Problemlösung diskutierten Annahmen zur Leistungsmotivation Atkinsons (1966), wonach sich erfolgsmotivierte und misserfolgsvermeidungsmotivierte Personen wie dargestellt auf unterschiedlichen Entscheidungsgrundlagen bewegen. Eine sichere Handlungsprognose lässt sich somit nicht aus dem Typologie-Ansatz ableiten.

Zudem lassen sich die Ausprägungen weder addieren noch multiplizieren, sondern sie sind als vier separat stehende Phänomene mit einem jeweils eigenen Wert $\mathrm{zu}$ respektieren und können in komplexer Weise aufeinander einwirken.

Last, not least lässt sich das Spinnendiagramm mit unterschiedlichen Wertesystematiken i. S. v. Bewertungsschemata hinterlegen und in der Folge kann überprüft werden, inwieweit sich ein Soll-Profil bzw. ein Handlungsspielraum normativ verändert, wenn ein anderes Bewertungsschema im Sinne eines anderen Wertekanons für die Bemessung eines Spielraums zugrunde gelegt wird. 


\subsection{Limitationen zur Persönlichkeitstypologie von Social Entrepreneuren}

In Erinnerung gerufen werden die Ausführungen zu Beginn von Kapitel $8 \mathrm{zu}$ Fertigkeiten (Skills) und Fähigkeiten (Abilities). Fertigkeiten können sich durch Lernprozesse stark verändern, so dass sie infolge aktueller, situativ gegebener Ereignisse und Befindlichkeiten das Kompetenzprofil einer Person prägen können. Dementsprechend lassen sich Fertigkeiten als sich kontinuierlich verändernde Zustände auffassen, wobei Veränderungen psychischer Zustände einzubeziehen sind (State-Ansatz).

In Abgrenzung hierzu lassen sich stabile Persönlichkeitsmerkmale mit den vorhandenen Fähigkeiten einer Person aufzeigen. Wenn hierbei davon ausgegangen wird, dass das Eigenschaftsprofil einer Person typische Verhaltensmuster dieser Person zu erkennen gibt, dann lassen sich Verhaltenstendenzen auf Basis von Persönlichkeitseigenschaften situationsunabhängig prognostizieren (Trait-Ansatz).

Wenngleich Motive mit Handlungszielen in Verbindung stehen (Nerdinger, 2013, S. 8; Schneider \& Schmalt, 2000), lassen sie sich aufgrund ihrer situationsübergreifend gültigen Wertungsdispositionen ebenfalls dem Trait-Ansatz zuordnen, demgemäß aus deren Erscheinungsform Handlungsprognosen abzuleiten sein sollten. In Abgrenzung hierzu verkörpern Bedürfnisse situationsspezifische, sich nicht nur kontinuierlich verändernde Befindlichkeiten, sondern durchaus schnell und plötzlich wechselnde Zustände einer Person (State-Ansatz). Da jedoch der vollständige Motivationsprozessablauf einen volitionalen Anteil beinhaltet, wird er dementsprechend im State-Ansatz verortet. In der Gesamtbetrachtung wird damit deutlich, dass Handlungsprognosen keinem einfachen Bedingungsgefüge unterliegen können.

Einerseits kann gemäß dem Trait-Ansatz davon ausgegangen werden, dass ein SE, der eine stark ausgeprägte Fähigkeit im Sinne einer Begabung im Umgang mit Zahlen besitzt und in der Folge eine schnelle und gute Auffassung beim kaufmännischen Rechnen zeigt, seine Buchhaltung gut bearbeiten kann. Andererseits belegen die Ausführungen zur Zielsetzung einen Kriterienkomplex, der individuell und situativ unterschiedlich ausgeprägt Einfluss nimmt. Darüber hinaus belegen die Ausführungen von Befragten, dass die Handlungsbereitschaft beispielsweise durch eigene Erfahrungswerte bzw. durch das erfahrungsbasierte Verhaltensrepertoire der betreffenden Person beeinflusst wird (IPk ${ }^{1080}$; IPi ${ }^{1081}$; IP28 ${ }^{1082}$ ) sowie durch die Bewertung der eigenen Kompetenzen im Hinblick auf deren Eignung, eine bestehende Absicht zu verfolgen (IPd ${ }^{1083}$; vertiefend dargestellt z. B. bei Nerdinger, 2013, S. 7 ff.). 
Nur auf den ersten Blick mag es daher naheliegend erscheinen, mithilfe des umfangreich vorliegenden Datenmaterials eine Typologie zu den Persönlichkeitsmerkmalen von SE als allgemeingültiges Modell herausarbeiten zu können, um auf diesem Weg z. B. Rückschlüsse auf Gestaltungsbedingungen für erfolgreiches Sozialunternehmertum ziehen zu können. Dass sowohl im eigenen Datenmaterial als auch in bereits vorliegenden qualitativen Untersuchungen vielfach Beispiele zu finden sind, die auf die Ausprägung von Persönlichkeitsmerkmalen von SE hindeuten, ist zudem tautologisch, da menschliches Verhalten grundsätzlich nicht losgelöst von Persönlichkeitsmerkmalen zum Ausdruck kommt. Ein Automatismus zur SE-Typisierung, geschweige denn mit empirisch abgesichertem Erkenntnisgewinn in eindeutiger Abgrenzung beispielsweise gegenüber KE, ergibt sich daraus noch nicht. Gleichwohl bestehen begründete Anhaltspunkte, dass SE sich in ihrer Persönlichkeitsausprägung von KE unterscheiden können (IPö ${ }^{1084}$ ). Daraus kann jedoch keineswegs abgeleitet werden, dass sich SE gegenüber KE durch das Vorhandensein bestimmter Persönlichkeitseigenschaften abgrenzen, die nur bei SE auftreten. Erinnert wird an die Ausführungen in Abschnitt 8.3 zum empirisch belegten Bedeutungsgehalt von Empathie bei SE; KE können ebenfalls empathisch sein und wie in Abschnitt 9.11 noch diskutiert, weist die FührungsstilForschung darauf hin, dass diese Eigenschaft ebenso für KE von Vorteil sein kann.

Wenngleich Persönlichkeitseigenschaften, Werte, Motive und Bedürfnisse Einfluss nehmen auf Handlungsentscheidungen und -ausführungen (z. B. Locke, Shaw, Saari \& Latham, 1981; Hollenbeck \& Brief, 1987; Sheldon \& Elliot, 1999; Judge, Bono, Erez \& Locke, 2005; Storch \& Krause, 2014) und dabei in Situationen eine erhöhte Handlungsbereitschaft besteht, wenn diese Situationen zur individuellen Ausprägung von Dispositionen der Persönlichkeit passen (Ickes, Snyder \& Garcia, 1997), lassen sich auf der Basis von identifizierten Persönlichkeitsmerkmalen keine kausal begründeten Zusammenhänge für eine Handlungsprognose ableiten. Dies bezieht sich gleichermaßen auf die Unmöglichkeit, verlässliche Vorhersagen für Erfolg und Misserfolg im Berufsleben zu treffen (vgl. z. B. Asendorpf, 2015, S. 22 ff.; für Entrepreneurship vgl. z. B. Leutner, Ahmetoglu, Akhtar \& Chamorro-Premuszic, 2014, m. V. a. Baron, Frese \& Baum, 2007; Zhao, Seibert \& Lumpkin, 2010; Zhao \& Seibert, 2006). So spricht sich Lazear $(2004 ; 2005)$ mit seinem Skill-Variety-Modell für eine Fertigkeitenvielfalt von Unternehmern aus und geht davon aus, dass sich dadurch die unternehmerische Aufgabenvielfalt erfolgreicher bewerkstelligen lässt; doch konnte dieser Zusammenhang nicht widerspruchsfrei bestätigt werden (zur Diskussion hierzu vgl. z. B. Krieger, 2017). Unternehmerpersönlichkeiten sind 
heterogen (Kerr, Kerr \& Xu, 2017) und den einen, passenden Persönlichkeitsschlüssel gibt es ebenso wenig wie das sozialunternehmerische Universalschloss des Erfolgs. Erkenntnisse aus Untersuchungen zur Situation von KE untermauern die Problematik: Beispielsweise hängt die Kundenkontaktgestaltung deutlich stärker von den Arbeitsanforderungen ab als von personenbezogenen Merkmalen (Neyer \& Asendorpf, 2018, S. 144; vgl. ebenso die Ausführung in Abschn. 8.12).

Zabel (2011) diskutiert im Rahmen einer Modellierung von Verhaltensdeterminanten für Nachhaltigkeit einen umfassenden Einfluss sowohl der soziokulturellen Umweltbedingungen als auch der Persönlichkeit eines Akteurs auf dessen Handeln. Faltin (2010) geht so weit und behauptet für Unternehmensgründer allgemeingültig, jedoch empirisch nicht belegt: „Heute hat jeder das Potenzial zum Gründer. Es stimmt nicht, dass Sie zum Unternehmer geboren sein müssen. Es stimmt nicht, dass Sie ein Patent haben sollten und viel Kapital brauchen. Es stimmt nicht, dass Sie detaillierte Kenntnisse der Betriebswirtschaftslehre, der Rechtsfragen, des Marketings oder der Finanzierung brauchen. Ich sage das nicht einfach leichtfertig dahin, sondern mit der Erfahrung und der Überzeugung aus 30 Jahren Beschäftigung mit dem Gründungsthema“" (ebd., S. 137). Doch werden mit diesem Statement nicht ansatzweise (Miss-)Erfolgsfaktoren für Entrepreneurship thematisiert. Jacobsen (2003) stellt hierzu die Hypothese auf: „Persönlichkeitsmerkmale wirken direkt und intensiv auf die Person des Entrepreneurs, aber nur indirekt auf den Erfolg und sind deshalb ein Einflussfaktor mit mittlerem Wirkungsgrad“" (S. 183).

Dennoch ist die Antwort auf die Frage nach der SE-spezifischen Persönlichkeitstypologie so einfach wie kompliziert zugleich, da bemerkenswerte Hinweise unterschiedlicher Art und Qualität im Hinblick auf entrepreneurbezogene Kompetenzanforderungen bestehen - obwohl abermals ohne Fokus auf Sozialunternehmertum. Wenngleich an dieser Stelle an die in Kapitel 2 thematisierte eingeschränkte Aussagekraft quantitativer Studien zur Intentionsbildung erinnert wird, u. a. bei fehlenden Longitudinalstudien (vgl. Kruse, Wach, Costa \& Moriano, 2018), können gerade auf Faktorenanalysen basierende Untersuchungserkenntnisse aus der Persönlichkeitsforschung herangezogen werden:

Optimalerweise lassen sich viele wechselseitig korrelierte Variablen in wenigen Dimensionen, sog. Faktoren, zusammenfassen. Dabei kann „man sich unter einem Faktor eine neue Variable vorstellen (...), die so gewählt ist, dass ihre Ähnlichkeit zu allen Variablen der Gruppe maximal ist. Erfassen die Variablen Eigenschaften, entsprechen die Faktoren breiteren Eigenschaften" (Neyer \& Asendorpf, 2018, S. 105). Eine überschaubare Anzahl an Eigenschaften soll die spezifische Ausprägung einer typischen Persönlichkeit erfassen. Daher sollten 
sich möglichst viele Items auf wenige unabhängige Faktoren reduzieren lassen, wobei bestimmte Korrelationsmuster spezifische Eigenschaftsdimensionen abbilden. Dargestellt werden konnten auf diesem Wege fünf unabhängige Dimensionen als Hauptfaktoren der Persönlichkeit (Borkenau \& Ostendorf, 2008; Costa \& McCrae, 1992; im Ursprung zurückgehend auf die lexikalischen Studienerkenntnisse von Allport \& Odbert, 1936), auch als „Big Five“ bezeichnet. Diese sind Neurotizismus, Extraversion, Offenheit für neue Erfahrungen, Verträglichkeit und Gewissenhaftigkeit. Deren Bedeutungsgehalt wird nachfolgend kurz skizziert, dabei werden Erkenntnisse aus der Entrepreneurship-Forschung eingebunden:

1) Im Hinblick auf Neurotizismus beschreibt Schwingenstein für die von ihr Befragten, dass negative Stellungnahmen Dritter gegenüber der sozialunternehmerischen Idee auf die Motivation des SE längerfristig beeinträchtigend einwirken können (2013, S. 57) und der Blick auf das bereits Erreichte zugunsten einer Defizitorientierung des eigenen Handelns vernachlässigt wird (ebd., S. 76 ff.). Damit bestehen Indikatoren für einen stark positiven NeurotizismusWert, der im Wesentlichen durch ausgeprägte, sich zudem kontinuierlich zeigende Ängstlichkeit, Verletzlichkeit, emotionale Instabilität, negative Überzeugungsmuster sowie soziale Befangenheit gekennzeichnet ist und für den Korrelationseffekte mit Burnout nachgewiesen sind (z. B. Bianchi, 2018; Bailey, 2017). Positiver Neurotizismus kann bei SE vorkommen und dann eine Herausforderung im beruflichen Handeln darstellen (vgl. z. B. Adegbuyi, Ayoade, Ogunnaike, Adegbuyi \& Binuomote, 2018; Kerr, Kerr \& Xu, 2017). Gleichwohl lassen sich im eigenen Datenmaterial markante Aussagen finden, die den Rückschluss auf einen negativen Neurotizismuswert bei erfolgreichen IP zulassen (IP24 ${ }^{1085}$; IPA ${ }^{1086}$; IPA ${ }^{1087} ;$ IPF $^{1088}$ ) und damit emotionale Stabilität, innere Ruhe und Ausgeglichenheit sowie soziale Unbefangenheit untermauern.

2) Extraversion und Introversion bilden die beiden Pole der gemeinsamen Dimension Extraversion. Extravertierte Personen schenken unterschiedlichen Akteuren ihres sozialen Umfelds aktiv Aufmerksamkeit, dies kommunikativ und mit dem kontinuierlich auftretenden Bedürfnis nach Stimulation im und durch das Umfeld (IP8 ${ }^{1089}$ ). Leutner, Ahmetoglu, Akhtar und Chamorro-Premuszic (2014) zeigen, dass Extraversion unternehmerisches Handeln begünstigt. Caliendo, Fossen und Kritikos (2014) untermauern dies speziell für das Networking von Entrepreneuren. Extraversion gilt als Prädiktor für Erfolg im Verkauf und Vertrieb und generell im Kundenkontakt sowie im Hinblick auf Führungsund Managementstärke (Barrick, Mount \& Judge, 2008; Christiansen \& Tett, 2008). 
3) Personen mit ausgeprägter Offenheit gegenüber neuen Erfahrungen erleben Routinen und vorgegebene Regelwerke als Einschränkung. Lord (2011) betont, dass selbstständiges Arbeiten, das ein Unternehmertum kennzeichnet, positiv erlebt wird, da Selbstbestimmtheit ausgelebt werden kann (ebenfalls bei Zhao $\&$ Seibert, 2006). Dies ist jedoch zu relativieren, wenn z. B. bei einem Mangel an Selbstorganisation, an Zeitmanagement, an strukturiertem und an zielorientiertem Arbeiten der Segen zum Fluch wird. Bezüglich des Faktors Offenheit für neue Erfahrungen werden kontroverse Diskussionen geführt (vgl. Borkenau \& Ostendorf, 2008, S. 10), wobei sich die jeweils vorliegende Kombination zu den weiteren Persönlichkeitsmerkmalen als mitentscheidend erweist bzw. eine Prognose verkomplizieren kann. Beispielsweise lässt sich für einen stark strukturierten und sicherheitsverbundenen SE, der offen gegenüber neuen Erfahrungen eingestellt ist und sozialunternehmerische Aktivitäten umsichtig plant, annehmen, dass das Sichern der Anfangsfinanzierung für ihn einen relativ hohen Stellenwert besitzt. Befragte zeigen auf, dass auf dieser Basis - gepaart mit hinreichend Risikobereitschaft - die Entscheidung, eine SEO zu gründen, gewissenhaft gefällt werden kann (IPI ${ }^{1090}$ ), wobei die Chancen, die sich ergeben, mit hinreichend Selbstvertrauen ergriffen werden (IPE ${ }^{1091}$ ).

4) Verträglichkeit verkörpert eine hohe, sich kontinuierlich bemerkbar machende Ausrichtung auf zwischenmenschliche Kontaktgestaltung mit fürsorglichen, auf das Gute im Gegenüber vertrauenden Komponenten und somit auf prosoziales Verhalten, das gesellschaftlich nützlich und wertschätzungswert ist (vgl. Dovidio, Piliavin, Schroeder \& Penner, 2006; ebenso z. B. bei IP6 ${ }^{1092}$ ). Der Gegenpol ist gekennzeichnet durch Egoismus, Missgunst und von Rücksichtslosigkeit geprägte Beziehungsgestaltung. Einerseits korreliert Verträglichkeit mit Teamerfolg (Hough \& Furnham, 2003). Andererseits stellen schwache Verträglichkeitswerte einen Indikator für erfolgreiches Unternehmertum dar, da Verhandlungen dann von interessengeleiteter Konsequenz und Stringenz geprägt sind (Zhao \& Seibert, 2006). IP4 sinniert hierzu passend, inwieweit SE empathischer sind als $\mathrm{KE}^{1093}$. Dies deckt sich mit den zuvor diskutierten Erkenntnissen (vgl. z. B. Ruskin, Seymour \& Webster, 2016; Lambrechts, Caniëls, Molderez, Venn \& Oorbeek, 2020). Im Übrigen scheint bei stark ausgeprägter Verträglichkeit denkbar, dass es für einen extravertierten SE mit deutlich positiv ausgeprägtem Neurotizismuswert keinesfalls zutreffen muss, was Maslow postuliert, nämlich ,,self-actualizing people do not need to be loved by everyone“ (Maslow, 1971, S. 298) sowie „self-actualizing people do not need or seek for or even enjoy very much flattery, applause, popularity, status, prestige, nomey, honors, etc.“ (Maslow, 1971, S. 299). 
5) Stark positiv ausgeprägte Gewissenhaftigkeit, gepaart mit stark negativ ausgeprägtem Neurotizismus, begünstigt es im Hinblick auf die Arbeitsleistung, einen höheren Schwierigkeitsgrad bei der Zielsetzung zu wählen (Judge \& Ilies, 2002; Hinsz \& Jundt, 2005). Ebenso neigen Menschen mit ausgeprägter Gewissenhaftigkeit eher dazu, sich unabhängig von Dritten Ziele zu setzen (vgl. Barrick, Mount \& Strauss, 1993), die zudem besonders anspruchsvoll sein können (vgl. Komarraju \& Karau, 2005). Für SE kann dabei gelten: Gerade wenn ein neuer Markt erschlossen werden muss und hierbei auf kein etabliertes Vorgehen zurückgegriffen werden kann, lässt sich eine Affinität zur Selbstausbeutung herleiten. Dabei besteht ein erhöhtes Gefährdungspotenzial für gewissenhaft arbeitende Unternehmer, die überzeugt sind, hohe Arbeitsleistungen erbringen zu können. Denn sie können dazu neigen, sich zu anspruchsvolle Ziele zu setzen, die im zeitlichen Verlauf infolge Überlastung zur Beeinträchtigung des Betroffenen führen - insbesondere dann, wenn sie als hoch wichtig und sehr dringlich priorisiert werden (Locke, Motowidlo \& Bobko, 1986; Hollenbeck \& Brief, 1987).

Kritisch ist anzumerken, dass Erkenntnisse aus Studien zu den Big Five inkongruent sind (vgl. z. B. Zhao \& Seibert, 2006; Hough \& Furnham, 2003). Beispielsweise testieren Leutner, Ahmetoglu, Akhtar \& Chamorro-Premuszic (2014) ebenfalls im Hinblick auf Verträglichkeit unternehmerischen Erfolg, vor allem für innovatives Entrepreneurship (ebd., S. 61 f.), wobei Innovation nicht hinreichend operationalisiert wird (ebd., S. 8). „Daneben können Alterseffekte wirken, so dass ältere Probanden im Vergleich zu jüngeren niedrigere Werte in Neurotizismus, Extraversion und Offenheit für Erfahrungen aufweisen, dafür eine höhere Verträglichkeit und Gewissenhaftigkeit“" (Meyer, 2020, S. 47, m. V. a. Borkenau \& Ostendorf, 2008). Zudem lässt sich keine kulturübergreifende Kongruenz nachweisen (zur Diskussion hierzu siehe z. B. Neyer \& Asendorpf, 2018; Meyer, 2020, S. 49 f.; Bipp, 2006, S. 14 ff.). „Angemessen erscheint vielmehr der Standpunkt des Kontextualismus, wonach die Vergleichbarkeit von Persönlichkeitseigenschaften und ihrer Messung oft gesichert werden kann, wenn zuvor ihre kulturelle Bedeutung empirisch untersucht wird und sie auf dieser Grundlage kulturell vergleichbar gemacht werden“ (Neyer \& Asendorpf, 2018, S. 420). Jedoch zeigen die unterschiedlichen Sichtweisen, beispielsweise im Hinblick auf die Verortung im unternehmerischen Denken und Handeln, dass es nicht die eine sozialunternehmerische Kultur gibt, nicht mal eine eng umrissene sozialunternehmerische Kulturlandschaft. Vielmehr kann gezeigt werden, dass eine Vielfalt an Kulturlandschaften besteht. 
In der Gesamtbetrachtung erweisen sich selbst die Big Five nicht als geeignet, um spezifische Persönlichkeitskonstrukte zu beschreiben (Meyer, 2020, S. 49). Weiterhin ist festzuhalten, ,there is a lack of consensus about what competencies are essential for the development of a career in social entrepreneurship" (Miller, Wesley \& Williams, 2012, S. 350). „Aus der Einsicht in die Vielfalt der Persönlichkeit (...) erwächst dagegen die Forderung, gerade nicht einen bestimmten Persönlichkeitstyp anzustreben, sondern die Vielfalt der Persönlichkeit zu achten und zu bewahren" (Neyer \& Asendorpf, 2018, S. 424). Bei der Vielfalt an sozialunternehmerischen Aktivitäten und ihren branchen- wie themenübergreifenden Einsatzfeldern, in denen auch sozial Benachteiligte ohne Lobby zu Sozialunternehmern werden, sollte als ethisches Prinzip somit beherzigt werden, Möglichkeiten zu schaffen und zu fördern, die allen Persönlichkeiten den Weg für sozialunternehmerisches Engagement ebnen.

\subsection{Typologie-Ansatz zum Anspruchsniveau: Problemlösung, Hochskalierbarkeit und Innovation}

Zuvor wurden Anspruchsniveaus zur sozialen Problemlösung, zur Skalierung und dabei insbesondere zur Hochskalierung sowie zur Innovationsentwicklung diskutiert, sodann der Stellenwert zur Zielsetzung und Zielerreichung und daraufhin die konzeptionelle Vereinbarkeit bzw. Unvereinbarkeit des Sozialen und des Unternehmerischen. Limitationen in der Entwicklung einer SE-typischen Persönlichkeitstypologie wurden ebenfalls genau betrachtet. In der Zusammenschau dieser Diskussionspunkte lässt sich ein weiterer Typologie-Ansatz herausarbeiten. Denn SE lassen sich im Hinblick auf ihre Anspruchsniveaus zu den drei verschiedenen Kriterien Problemlösung, Hochskalierung und Innovieren typologisieren. Dabei können die drei Kriterien zu einem gemeinsamen Bild zusammengesetzt werden, so dass SE-typenbezogene Aussagen getroffen werden können. In der Gesamtbetrachtung entstehen 18 denkbare SE-Typen mit jeweils unterschiedlichen Ausprägungen in ihren Anspruchsniveaus. Zur methodischen Anregung für die Typenentwicklung wurde auf den Fragebogen zur Erhebung von Arbeitszufriedenheitstypen von Ferreira (2009) zurückgegriffen.

Erkennbar wird, dass SE-Typen, die sich höhere Ziele setzen, als sie erreichen können, potenziell gefährdeter sind bzw. besondere Bewältigungsstrategien benötigen, woraus insbesondere ein entsprechend höheres Erfordernis an psychischer Widerstandsfähigkeit (Resilienz) abgeleitet wird.

Tabelle 8.2 skizziert die 18 verschiedenen SE-Typen. Mit einer Ampelsystematik wird das zunehmende Gefährdungspotenzial dargestellt, wobei neben den 
gängigen drei Ampelfarben zusätzlich mit Orange eine Zwischenstufe für deutlich erhöhtes Gefährdungspotenzial eingeführt wird.

Zur Erläuterung von Tabelle 8.2: Die erste Spalte kennzeichnet die verschiedenen Typen von 1 bis 18. Dabei nimmt das Resilienz-Erfordernis zu: Typ 1 besitzt kein besonderes Resilienz-Erfordernis und Typ 18 besitzt das höchste Resilienz-Erfordernis. Die zweite Spalte zeigt das Anspruchsniveau für soziale Problemlösung (PL), die dritte Spalte für Hochskalierung (HS) und die vierte Spalte für Innovieren (IV). Die fünfte Spalte beinhaltet Kurzbeschreibungen für den jeweiligen Typus.

Jedes der drei Kriterien ist in seinem Anspruchsniveau theoretisch in drei verschiedenen Ausprägungen denkbar. Dabei repräsentiert der positive Zahlenwert „1“, dass der SE bezogen auf das entsprechende Kriterium keine Zielsetzung verfolgt, sein Anspruchsniveau intrapsychisch also keinen negativen Druck erzeugt. Eine „1“ bei „Problemlösung“ repräsentiert demnach, dass ein SE in seinem eigenen definitorischen Verständnis zum Sozialunternehmertum nicht die Zielsetzung verankert, dass Social Entrepreneure ein soziales Problem lösen müssen. Beispielsweise ist denkbar, dass der entsprechende SE den Anspruch hat, seinen sozialunternehmerischen Beitrag zu leisten, um einen bestehenden Missstand zur sozialen Benachteiligung zu reduzieren. Er bereitet sich also keinerlei Druck, ein Problem eliminieren zu müssen. Dieses Anspruchsniveau wird deswegen mit dem positiven Zahlenwert „1“ gekennzeichnet, da eine solche Selbstbewertung keine Fehlbelastung und somit keine negative Beanspruchung nach sich ziehen kann. Die sich daraus ergebende Konsequenz ist somit positiv zu bewerten. Dementsprechend bedeutet eine „1“ bei Hochskalierung, dass sich der entsprechende SE keinen Druck setzt, seine SEO hochskalieren zu müssen - unabhängig davon, was er unter Hochskalierung versteht. Eine „1“ bei Innovieren bedeutet somit, dass sich der betreffende SE nicht unter Druck setzt, eine Innovation mit seinem sozialunternehmerischen Engagement hervorbringen zu müssen - unabhängig davon, was er unter Innovation bzw. Innovieren versteht. Dass im Praxisfeld sehr unterschiedliche, sich widersprechende Begriffsverständnisse vorliegen, wurden weiter oben thematisiert. An dieser Stelle geht es jedoch lediglich um die Frage, ob ein Anspruchsniveau vorliegt, nicht aber darum, wie dieses im Einzelnen inhaltlich ausgestaltet ist.

Der negative Zahlenwert „-1“ kennzeichnet, dass für das jeweilige Kriterium ein zu hohes Anspruchsniveau besteht und demzufolge das damit verbundene Ziel nicht erreicht wird. Ein SE setzt sich also ein zu anspruchsvolles Ziel. Eine „-1“ bei Problemlösung bedeutet somit, dass der betreffende SE sich das Ziel setzt, mit seinem sozialunternehmerischen Engagement einen Missstand zur sozialen 
Tabelle 8.2 18 SE-Typen zum Anspruchsniveau: Problemlösung, Hochskalierbarkeit und Innovation. (eigene Darstellung)

\begin{tabular}{|c|c|c|c|c|}
\hline $\begin{array}{c}\text { Typ- } \\
\text { Nr. }\end{array}$ & $P L$ & HS & IV & Kurzbeschreibung \\
\hline 1 & 1 & 1 & 1 & SE setzt sich nicht unter Druck; es besteht daher kein besonderes Resilienz-Erfordernis \\
\hline 2 & 1 & 1 & 0 & $\begin{array}{l}\text { SE setzt sich teilweise unter Druck, wird allerdings seinem Anspruchsdenken an } \\
\text { Innovation gerecht; es besteht kein besonderes Resilienz-Erfordernis; Innovation ist } \\
\text { aber je nach Wettbewerbssituation und Marktentwicklung nicht von Dauer gegeben, so } \\
\text { dass dennoch Vorsicht geboten ist }\end{array}$ \\
\hline 3 & 1 & 0 & 1 & $\begin{array}{l}\text { SE setzt sich teilweise unter Druck, wird allerdings seinem Anspruchsdenken an } \\
\text { Hochskalierung gerecht; es besteht kein besonderes Resilienz-Erfordernis; } \\
\text { Hochskalierung kann aber Abhängigkeiten erhöhen, so dass dennoch Vorsicht geboten } \\
\text { ist }\end{array}$ \\
\hline 4 & 1 & 0 & 0 & $\begin{array}{l}\text { SE setzt sich deutlich unter Druck, wird allerdings seinem Anspruchsdenken an } \\
\text { Hochskalierung und Innovation gerecht; es besteht kein besonderes Resilienz- } \\
\text { Erfordernis; Innovation ist aber je nach Wettbewerbssituation und Marktentwicklung } \\
\text { nicht von Dauer gegeben und Hochskalierung kann Abhängigkeiten erhöhen, so dass } \\
\text { dennoch Vorsicht geboten ist }\end{array}$ \\
\hline 5 & 1 & 1 & -1 & $\begin{array}{l}\text { SE setzt sich teilweise unter Druck und wird seinem Anspruchsdenken an Innovation } \\
\text { nicht gerecht; es besteht ein besonderes Resilienz-Erfordernis! }\end{array}$ \\
\hline 6 & 1 & 0 & -1 & $\begin{array}{l}\text { SE setzt sich deutlich unter Druck; er wird zwar seinem Anspruchsdenken an } \\
\text { Hochskalierung gerecht, was für Erfolg spricht, aber besonders unter Druck setzen } \\
\text { kann; er wird seinem Anspruchsdenken an Innovation nicht gerecht; es besteht ein } \\
\text { besonderes Resilienz-Erfordernis! }\end{array}$ \\
\hline 7 & -1 & 1 & 1 & $\begin{array}{l}\text { SE setzt sich zwar nur teilweise unter Druck, wird aber seinem Anspruchsdenken an } \\
\text { Problemlösung nicht gerecht; es besteht ein besonderes Resilienz-Erfordernis! }\end{array}$ \\
\hline 8 & 1 & -1 & 1 & $\begin{array}{l}\text { SE setzt sich zwar nur teilweise unter Druck, wird aber seinem Anspruchsdenken an } \\
\text { Hochskalierung nicht gerecht; es besteht ein besonderes Resilienz-Erfordernis! }\end{array}$ \\
\hline 9 & -1 & 1 & 0 & $\begin{array}{l}\text { SE setzt sich deutlich unter Druck; er wird zwar seinem Anspruchsdenken an Innovation } \\
\text { gerecht, nicht aber seinem Anspruchsdenken an Problemlösung; Innovation ist je nach } \\
\text { Wettbewerbssituation und Marktentwicklung nicht von Dauer gegeben; es besteht ein } \\
\text { besonderes Resilienz-Erfordernis! }\end{array}$ \\
\hline 10 & 1 & -1 & 0 & $\begin{array}{l}\text { SE setzt sich deutlich unter Druck; er wird zwar seinem Anspruchsdenken an Innovation } \\
\text { gerecht, nicht aber seinem Anspruchsdenken an Hochskalierung; Innovation ist je nach } \\
\text { Wettbewerbssituation und Marktentwicklung nicht von Dauer gegeben; es besteht ein } \\
\text { besonderes Resilienz-Erfordernis! }\end{array}$ \\
\hline 11 & -1 & 0 & 1 & $\begin{array}{l}\text { SE setzt sich deutlich unter Druck; er wird zwar seinem Anspruchsdenken an } \\
\text { Hochskalierung gerecht, was für Erfolg sprechen kann, aber zusätzlichen Druck } \\
\text { bereiten kann; er entspricht aber nicht seinem Anspruchsdenken an Problemlösung; es } \\
\text { besteht ein besonderes Resilienz-Erfordernis! }\end{array}$ \\
\hline 12 & -1 & 0 & 0 & $\begin{array}{l}\text { SE setzt sich deutlich unter Druck; er wird seinem Anspruchsdenken an Problemlösung } \\
\text { nicht gerecht; er wird jedoch seinem Anspruchsdenken an Innovation und } \\
\text { Hochskalierung gerecht; Innovation ist je nach Wettbewerbssituation und } \\
\text { Marktentwicklung nicht von Dauer gegeben; Hochskalierung kann für Erfolg sprechen, } \\
\text { aber ebenso zusätzlich unter Druck setzen; es besteht ein besonderes Resilienz- } \\
\text { Erfordernis! }\end{array}$ \\
\hline
\end{tabular}

(Fortsetzung) 
Tabelle 8.2 (Fortsetzung)

\begin{tabular}{|c|c|c|c|c|}
\hline $\begin{array}{c}\text { Typ- } \\
\text { Nr. }\end{array}$ & $P L$ & HS & IV & Kurzbeschreibung \\
\hline 13 & 1 & -1 & -1 & $\begin{array}{l}\text { SE setzt sich deutlich unter Druck; er wird weder seinem Anspruchsdenken an } \\
\text { Innovation gerecht noch seinem Anspruchsdenken an Hochskalierung; daher } \\
\text { besteht ein auffallend hohes Resilienz-Erfordernis; SE kann psychisch besonders } \\
\text { stark gefährdet sein! }\end{array}$ \\
\hline 14 & -1 & 1 & -1 & $\begin{array}{l}\text { SE setzt sich deutlich unter Druck; er wird weder seinem Anspruchsdenken an } \\
\text { Problemlösung gerecht noch seinem Anspruchsdenken an Innovation; daher } \\
\text { besteht ein auffallend hohes Resilienz-Erfordernis; SE kann psychisch besonders } \\
\text { stark gefährdet sein! }\end{array}$ \\
\hline 15 & -1 & -1 & 1 & $\begin{array}{l}\text { SE setzt sich deutlich unter Druck; er wird weder seinem Anspruchsdenken an } \\
\text { Problemlösung gerecht noch seinem Anspruchsdenken an Hochskalierung; es } \\
\text { besteht ein auffallend hohes Resilienz-Erfordernis; SE kann psychisch besonders } \\
\text { stark gefährdet sein! }\end{array}$ \\
\hline 16 & -1 & 0 & -1 & $\begin{array}{l}\text { SE besitzt ein extrem ausgeprägtes Anspruchsdenken und setzt sich auffallend } \\
\text { stark unter Druck; er wird dabei weder seinem Anspruchsdenken an } \\
\text { Problemlösung gerecht noch seinem Anspruchsdenken an Innovation; er wird } \\
\text { zwar seinem Anspruchsdenken an Hochskalierung gerecht, was für Erfolg } \\
\text { sprechen kann. Dieser Erfolg kann aber zusätzlich unter Druck setzen; es besteht } \\
\text { ein ausgesprochen hohes Resilienz-Erfordernis; der SE ist psychisch als } \\
\text { besonders stark gefährdet einzuschätzen! }\end{array}$ \\
\hline 17 & -1 & -1 & 0 & $\begin{array}{l}\text { SE besitzt ein extrem ausgeprägtes Anspruchsdenken und setzt sich extrem } \\
\text { unter Druck; er wird lediglich seinem Anspruch an Innovation gerecht, die aber je } \\
\text { nach Wettbewerbssituation und Marktentwicklung nicht von Dauer gegeben ist; er } \\
\text { wird allerdings weder seinem Anspruchsdenken an Problemlösung gerecht noch } \\
\text { seinem Anspruchsdenken an Hochskalierung; insgesamt besteht ein } \\
\text { ausgesprochen hohes Resilienz-Erfordernis, der SE ist psychisch als besonders } \\
\text { stark gefährdet einzuschätzen! }\end{array}$ \\
\hline 18 & -1 & -1 & -1 & $\begin{array}{l}\text { SE besitzt ein extrem ausgeprägtes Anspruchsdenken und setzt sich auffallend } \\
\text { stark unter Druck; er wird weder seinem Anspruchsdenken an Problemlösung } \\
\text { gerecht noch seinem Anspruchsdenken an Hochskalierung noch seinem } \\
\text { Anspruchsdenken an Innovation; insgesamt besteht ein ausgesprochen hohes } \\
\text { Resilienz-Erfordernis, der SE ist psychisch als besonders stark gefährdet } \\
\text { einzuschätzen! }\end{array}$ \\
\hline
\end{tabular}

Benachteiligung beseitigen zu müssen; allerdings schafft er dies nicht. Dementsprechend steht eine ,-1“ bei Innovieren dafür, dass im Anspruchsdenken des SE in jedem Fall von Social Entrepreneuren eine Innovation hervorgebracht werden muss, er selbst es jedoch mit seinem sozialunternehmerischen Engagement nicht 
geschafft hat bzw. nicht schafft. Eine ,-1" bei Hochskalierung bedeutet somit, dass ein SE seine SEO nicht hochskalieren kann, obwohl in seinem Anspruchsdenken Social Entrepreneure Hochskalierungen erreichen müssen.

Der Nullwert kennzeichnet, dass der SE für das entsprechende Kriterium ein hohes Anspruchsniveau anstrebt, das entweder darin liegt, Probleme zu lösen, Innovation hervorzubringen oder hochzuskalieren, und dass er seine damit verbundene Zielsetzung erreicht bzw. erreicht hat. Ist es dem SE z. B. gelungen, das, was er unter Hochskalierung versteht, auch umzusetzen, wird eine „0“ gesetzt. Der Nullwert wird deshalb gewählt, um damit die Nähe sowohl zum positiven als auch zum negativen Ausprägungsgrad zu verdeutlichen. Denn einerseits kann z. B. davon ausgegangen werden, dass der SE sein Hochskalieren als Erfolgserlebnis bewertet, was eine positive Konsequenz repräsentiert. Andererseits zeigt die Datenauswertung, dass erreichte Hochskalierung in besonderer Form zu Überforderungen und Fehlbelastungen führen kann, da mit Hochskalierung besondere Herausforderungen an den SE gestellt werden, die er eventuell nicht bewältigen kann. Dies repräsentiert eine negative Konsequenz. Ein Nullwert zeigt somit, dass eine Habachtstellung geboten ist. Vergleichbar verhält es sich beim Innovieren, wenn z. B. der Druck besteht, zur sozialunternehmerischen Behauptung am Marktgeschehen kontinuierlich innovativ bleiben zu müssen.

Allerdings wurde zuvor diskutiert, dass kein Befragter seine soziale Problemlösung erreicht hat und dass darüber hinaus kein SE identifiziert werden konnte, der dies geschafft hat. Aus Gründen der Zweckmäßigkeit wird an dieser Stelle die kontingente Entscheidung getroffen, eine erreichte Problemlösung bei der Typologie nicht weiter zu berücksichtigen. Dies würde sich nur hochtheoretisch als zweckmäßig erweisen, widerspricht aber den Untersuchungsergebnissen. Aus diesem Grund kann für Problemlösung nur eine „1“ oder eine ,"-1“ vergeben werden.

In der Übersicht bestehen somit folgende Bewertungsalternativen in der zu berücksichtigenden Typologie-Bildung:

- Sozialer Problemlösungsanspruch ist kein sozialunternehmerisches Muss im eigenen Anspruchsniveau (=1)

- Sozialunternehmer müssen in jedem Fall ein soziales Problem lösen, aber dieses Ziel wird nicht erreicht $(=-1)$

- Innovieren ist kein sozialunternehmerisches Muss im eigenen Anspruchsniveau $(=1)$

- Innovieren ist ein Muss im eigenen Anspruchsniveau und die damit verbundene Zielsetzung wird erreichbar gestaltet $(=0)$ 
- Sozialunternehmer müssen in jedem Fall innovieren, aber dieses Ziel wird nicht erreicht $(=-1)$

- Hochskalierung ist kein sozialunternehmerisches Muss im eigenen Anspruchsniveau $(=1)$

- Hochskalierung ist ein Muss im eigenen Anspruchsniveau und die damit verbundene Zielsetzung wird erreichbar gestaltet $(=0)$

- Sozialunternehmer müssen in jedem Fall hochskalieren, aber dieses Ziel wird nicht erreicht $(=-1)$

Somit stellt eine „1“ keinen negativen Stressor dar, eine „0“ eventuell eine positive Anregung, dies jedoch vorübergehend bzw. instabil, und eine ,-1“ einen mit hoher Wahrscheinlichkeit sogar stabilen negativen Stressor.

\section{Implikation des Typologie-Ansatzes für die Praxis und kritische Reflexion}

Dieser Typologie-Ansatz lässt sich in vergleichbarer Form zu der im Abschnitt 8.12 vorgestellten Ausarbeitung zur Vereinbarkeit des Sozialen und des Unternehmerischen heranziehen für Fragestellungen zur SE-bezogenen Situationsanalyse. Insbesondere lassen sich die Erkenntnisse im Praxisfeld nutzen für die Entwicklung von Beratungskonzeptionen: beispielsweise im Rahmen von Supervisionssitzungen, Coachings und Mentorings sowie in Workshops mit Selbstreflexionsanteilen, um SE typbezogen bzw. Teamstrukturen bedarfsorientiert beraten und begleiten zu können und mithilfe der Merkmalsausprägungen zu den Anspruchsniveaus Widersprüchlichkeiten ebenso wie Kongruenzen aufzudecken, moralische Dilemmata aufzuarbeiten, ein Unternehmensleitbild mit seiner Missions-Visions-Verankerung zu entwickeln oder beispielsweise Wirkungsmessungen zu spezifizieren. Das jeweilige Verständnis zu Hochskalierung, Innovation und Problemlösung ist dabei unumgänglich zu konkretisieren, was ebenfalls einen Nutzeneffekt für weitere sozialunternehmerische Vorhaben darstellt.

Eine Verbindung der beiden in dieser Dissertation entwickelten TypologieAnsätze wird bewusst vermieden, um den jeweils eigenen Wirkungsgehalt, der bei den beiden Ansätzen in unterschiedlicher Qualität gegeben ist, nicht zu verwässern. Zudem ist die herangezogene Skalierungsqualität des Typologie-Ansatzes zur Vereinbarkeit des Sozialen mit dem Unternehmerischen (schwach - mittel - stark) nicht mit der Skalierungsqualität in diesem Typologie-Ansatz vereinbar.

Bei der Entwicklung des Typologie-Ansatzes zu den Anspruchsniveaus stellte sich die Frage, inwieweit Wirkungsmessung ein eigenes, viertes Kriterium abbilden kann. Der zum Ausschluss führende Unterschied zu den drei inkludierten Kriterien liegt darin, dass ein hohes Anspruchsniveau zum Hochskalieren, zur 
Problemlösung und zum Innovieren von vornherein im Widerspruch zu sozialunternehmerischen Aktivitäten stehen kann. Hingegen sind Wirkungsmessungen für jedwede sozialunternehmerische Aktivität prinzipiell möglich, wenngleich sie bei ihrer Entwicklung in ihrer inhaltlichen Ausgestaltung und damit zur Sicherung der Machbarkeit an die jeweilige SEO-Zielsetzung anzupassen sind. Zwar können strukturell bedingte Hinderungsgründe wie z. B. fehlende Finanzierungsmöglichkeiten für Wirkungsmessungsentwicklungen vorliegen. Allerdings ist davon auszugehen, dass dies keine vergleichbare Fehlbelastung provoziert. Zudem konnte dem ausgewerteten Datenmaterial nicht entnommen werden, dass ein fehlender Wirkungsnachweis kategorisch zum Ausschluss von Sozialunternehmertum führt.

Dennoch ist eine Erweiterung der Typologie denkbar, beispielsweise zum Anspruchsniveau der Förderbedingungen. Insofern sollte dieser Typologie-Ansatz als flexible, anpassungsfähige Hilfsstruktur aufgefasst und genutzt werden.

Je nach Fragestellung und Zielsetzung, ob zu wissenschaftlichen Zwecken oder im Praxisfeld, erscheint es machbar, die Erhebung zu den kriterienbezogenen Anspruchsniveaus quantitativ empirisch auszuarbeiten. In jedem Fall muss dafür eine gewissenhafte SE-bezogene Operationalisiersung zu den drei Kriterien Problemlösung, Innovation und (Hoch-)Skalierung vorgenommen werden. Eventuell lässt sich sodann das Ergebnis mit validen Testverfahren kombinieren. Gegebenenfalls erscheint beispielsweise zur Ermittlung der SE-bezogenen Arbeitszufriedenheit eine Triangulation mit dem Fragebogen zur Erhebung von Arbeitszufriedenheitstypen von Ferreira (2009) denkbar.

Ebenso lassen sich unter Einbezug dieses Typologie-Ansatzes die bestehenden, sich stark unterscheidenden Definitionsansätze zum Social Entrepreneurship neu verorten.

\subsection{Verortung der Erkenntnisse im Wirkungsmodell zur Arbeitsgestaltung von Social Entrepreneuren}

Mit den in diesem Kapitel diskutierten Teilergebnissen lässt sich somit dreierlei aufzeigen:

Erstens: Personenbezogene Merkmale von SE, deren Einstellungen und Motive setzen Rahmenbedingungen zur Arbeitsgestaltung von SE. Diese Rahmenbedingungen wirken dementsprechend als eigenständiges Kriterium (im Wirkungsmodell wiedergegeben als Wirkungskriterium IV).

Zweitens: Einstellungen (z. B. eine Abneigung gegenüber der Auseinandersetzung mit Zahlen und betriebswirtschaftlichen Kennzahlen), Bedürfnisse (z. B. 
das Streben nach Transzendenz) und Motive (z. B. das dauerhafte Streben nach sozialer Anerkennung) wirken dynamisch in Entscheidungsprozessen direkt auf das Beanspruchungsgefüge, das SE erleben (im Wirkungsmodell wiedergegeben als Wirkungspfad 4).

Drittens: Mit ihren Einstellungen und Motiven prägen SE ihre Arbeits- und Lernkultur. So beeinflusst beispielsweise ein hoher Stellenwert des Unternehmertums im Verhältnis zur sozialen Zielsetzung die Kontaktgestaltung zu und Vernetzung mit anderen Akteuren qualitativ wie quantitativ (im Wirkungsmodell wiedergegeben als Wirkungspfad b; eine ausführliche Betrachtung arbeits- und lernkultureller Besonderheiten erfolgt in Kap. 9).

\section{Literaturverzeichnis}

Abebe, M. Kimakwa, S. \& Redd, T. (2020). Toward a typology of social entrepreneurs. The interplay between passionate activism and entrepreneurial expertise. In: Journal of Small Business and Enterprise Development, 27 (4), S. 509-530.

Ach, N. (1910). Über den Willen. Vortrag, gehalten in der gemeinschaftlichen Sitzung beider Hauptgruppen der 82. Versammlung Deutscher Naturforscher und Ärzte, in erweiterter Form dargestellt. Untersuchungen zur Psychologie und Philosophie, erster Band, erstes Heft. Leipzig: von Quelle \& Meyer.

Achtziger, A. \& Gollwitzer, P. (2010). Motivation und Volition im Handlungsverlauf. In: J. Heckhausen und H. Heckhausen (Hrsg.), Motivation und Handeln (4. Auflage). Berlin: Springer, S. 309-355.

Adegbuyi, O., Ayoade, E., Ogunnaike, O., Adegbuyi, A. \& Binuomote, M. (2018). The Impact of Neurotic Entrepreneurship on Business Performance of Small and Medium Scale Enterprises. Conference Paper, 12. International Technology, Education and Development Conference (INTED, 5.-7.3.2018), Valencia (Spanien).

Adro, F. do \& Fernandes, C. (2020). Social innovation: a systematic literature review and future agenda research. In: International Review on Public and Nonprofit Marketing, 17, S. $23-40$.

Allport, G. \& Odbert, H. (1936). Trait names. A psycho-lexical study. In: Psychological Monographs, 47 (1), Nr. 211.

Ambati, N. (2020). Motivating factors of social entrepreneurs: Evidences from Gujarat, India. Materials today: Proceedings. Abrufbar auf: https://www.sciencedirect.com/science/art icle/pii/S2214785320369832?via\%3Dihub. Abgerufen am: 22.12.2020.

Asendorpf, J. (2015). Persönlichkeitspsychologie für Bachelor (3., aktualisierte Auflage). Heidelberg: Springer Medizin Verlag.

Atkinson, J. (1966). Motivational Determinants of risk-taking behavior. In: J. Atkinson und N. Feather (Hrsg.), A Theory of Achievement Motivation. New York (USA): Wiley, S.11-29. Austin, J., Stevenson, H. \& Wei-Skillern, J. (2006). Social and Commercial Entrepreneurship: Same, Different, or Both? In: Entrepreneurship Theory and Practice, 30 (1), S. 1-22. 
Bacq, S. \& Janssen, F. (2011). The Multiple Faces of Social Entrepreneurship: A Review of Definitional Issues Based on Geographical and Thematic Criteria. In: Entrepreneurship \& Regional Development, 23 (5-6), S. 373-403.

Baeckmann, S. v. (1998). Downsizing. Zwischen unternehmerischer Notwendigkeit und individueller Katastrophe. München: Rainer Hampp.

Bailey, T. (2017). Executive functions as moderators of the neuroticism-burnout relationship. PhD Thesis. Nottingham (Großbritannien): University of Nottingham.

Bandura, A. (1997). Self-efficacy: The exercise of control. New York (USA): Freeman.

Bandura, A., \& Locke, E. (2003). Negative self-efficacy and goal effects revisited. In: Journal of Applied Psychology, 88, S. 87-99.

Baron, R., Frese, M. \& Baum, J. (2007). Research Gains: Benefits of closer links between I/O psychology and entrepreneurship. In: J. Baum, M. Frese und R. Baron (Hrsg.), The psychology of entrepreneurship. Mahwah (USA): Erlbaum, S. 347-373.

Barrick, M., Mount, M. \& Strauss, J. (1993). Conscientiousness and performance of sales representatives: Test of the mediating effects of goal setting. Journal of Applied Psychology, 78, S. 715-722.

Barrick, M., Mount, M. \& Judge, T. (2008). Personality and Job Performance at the Beginning of the New Millenium: What Do We Know and Where Do We Go Next? In: International Journal of Selection and Assessment, 9, S. 9-30.

Battilana, J. \& Lee, M. (2014). Advancing Research on Hybrid Organizing - Insights from the Study of Social Enterprises. In: Academy of Management Annals, 8 (1), S. 397-441.

Bechmann, G. \& Grunwald, A. (1998). Was ist das Neue am Neuen, oder: wie innovativ ist Innovation? In: Zeitschrift für Technikfolgenabschätzung in Theorie und Praxis, 7 (1), S. 4-11.

Beckmann, M. (2011). Social Entrepreneurship. Altes Phänomen, neues Paradigma moderner Gesellschaften oder Verbote eines Kapitalismus 2.0? In: H. Hackenberg und S. Empter (Hrsg.), Social Entrepreneurship - Social Business. Für die Gesellschaft unternehmen. Wiesbaden: Springer Fachmedien, S. 67-85.

Bertelsmann Stiftung (Hrsg.) (2013). Skalierung sozialer Wirkung. Handbuch zu Strategien und Erfolgsfaktoren von Sozialunternehmen. Gütersloh: Verlag Bertelsmann Stiftung.

Bianchi, R. (2018). Burnout is more strongly linked to neuroticism than to workcontextualized factors. In: Psychiatry Research, 270, S. 901-905.

Bipp, T. (2006). Persönlichkeit - Ziele - Leistung: Der Einfluss der Big Five Persönlichkeitseigenschaften auf das zielbezogene Leistungshandeln. Dissertation an der Universität Dortmund, Fakultät 14 Humanwissenschaften und Theologie, Institut für Psychologie, Lehrstuhl für Angewandte Organisationspsychologie.

Blickle, G. (2019a). Berufswahl und berufliche Entwicklung. In: F. Nerdinger, G. Blickle und N. Schaper (Hrsg.), Arbeits- und Organisationspsychologie (4., vollständig überarbeitete Auflage). Berlin: Springer, S. 209-234.

Boluk, K. \& Mottiar, Z. (2014). Motivations of social entrepreneurs. Blurring the social contribution and profits dichotomy. In: Social Enterprise Journal, 10 (1), S. 53-68.

Borkenau, P. \& Ostendorf, F. (2008). NEO-Fünf-Faktoren-Inventar nach Costa und Mc Crae (2., neu normierte und vollständig überarbeitete Auflage). Göttingen: Hogrefe.

Brieger, S., De Clercq, D. \& Meynhardt, T. (2020). Doing Good, Feeling Good? Entrepreneurs' Social Value Creation Beliefs and Work-Related Well-Being. Journal of Business Ethics. DOI: https://doi.org/10.1007/s10551-020-04512-6. 
Brooks, H. (1982). Social and Technical Innovation. In: S. Lundsted und E. Colglazier (Hrsg.), Managing Innovation. In: The Social Dimension of Creativity, Invention and Technology. New York (USA): Pergamon, S. 1-30.

Brunstein, J., Schultheiss, O. \& Grässmann, R. (1998). Personal Goals and emotional wellbeing: The moderating role of motive dispositions. In: Journal of Personality and Social Psychology, 75 (2), S. 494-508.

Caliendo, M., Fossen, F. \& Kritikos, A. (2014). Personality characteristics and the decisions to become and stay self-employed. In: Small Business Economics, 42 (4), S. 787-814.

Casini, A., Bensliman, R., Fossati, E., Degavre, F. \& Mahieu, C. (2018). Is Social Innovation Fostering Satisfaction and Well-Being at Work? Insights from Employment in Social Enterprises Providing Long-Term Eldercare Services. In: International Journal of Voluntary and Nonprofit Organizations, 29, S. 1244-1260.

Chell, E., Spence, L., Perrini, G. \& Harris, J. (2016). Social Entrepreneurship and Business Ethics: Does Social Equal Ethical? In: Journal of Business Ethics, 133, S. 619-625.

Christiansen, N. \& Tett, R. (2008). Toward a Better Understanding of the Role of Situations in Linking Personality, Work Behavior, and Job Performance. In: Industrial and Organizational Psychology, 1, S. 312-316.

Clark, K., Newbert, S. \& Quigley, N. (2018). The motivational drivers underlying for-profit venture creation: Comparing social and commercial entrepreneurs. In: International Small Business Journal: Researching Entrepreneurship, 36 (2), S. 220-241.

Coff, R. \& Kryscynski, D. (2011). Invited Editorial: Drilling for Micro-Foundations of Human Capital-Based Competitive Advantages. In: Journal of Management, 37 (5), S. 1429-1443.

Costa, P. \& McCrae, R. (1992). Revised NEO Personality Inventory (NEO-PI-R) and NEO Five-Factor Inventory (NEO-FFI). Professional manual. Odessa (USA): Psychological Assessment Resources.

Deci, E., Connell, J. \& Ryan, R. (1989). Self-Determination in a Work Organization. In: Journal of Applied Psychology, 74 (4), S. 580-590.

Deci, E., Koestner, R. \& Ryan, R. (1999). A Meta-Analytic Review of Experiments Examining the Effects of Extrinsic Rewards on Intrinsic Motivation. In: Psychological Bulletin, 125 (6), S. 627-668.

Deci, E. \& Ryan, R. (2000). The "What" and "Why" of Goal Pursuits: Human Needs and the Self-Determination of Behavior. In: Psychological Inquiry, 11 (4), S. 227-268.

Diochon, M. \& Anderson, A. (2010). Ambivalence and ambiguity in social enterprise; narratives about values in reconciling purpose and practices. In: International Entrepreneurship and Management Journal, 7, S. 93-109.

Dovidio, J., Piliavin, J., Schroeder, D. \& Penner, L. (2006). The Social Psychology of Prosocial Behavior. Mahwah (USA): Lawrence Erlbaum.

Drucker, P. (1957). Landmarks of tomorrow. New York (USA): Harper \& Row.

Earley, P. (1988). Computer-generated performance feedback in the magazine-subscription industry. In: Organizational Behavior and Human Decision Processes, 41 (1), S. 50-64.

Earley, P., Wojnaroski, P. \& Prest, W. (1987). Task planning and energy expended: Exploration of how goals influence performance. In: Journal of Applied Psychology, 71, S. 107-114.

Emmons, R. (1992). Abstract versus concrete goals: Personal striving level, physical illness, and psychological well-being. In: Journal of Personality and Social Psychology, 62, S. 292-300. 
Erpf, P., Tekula, R. \& Neuenschwander, J. (2019). Clustering social enterprises: an empirically validated taxonomy. In: Social Enterprise Journal, 15 (3), S. 397-420.

Faltin, G. (2010). Kopf schlägt Kapital. Von der Lust, ein Entrepreneur zu sein. In: J. Galuska (Hrsg.), Die Kunst des Wirtschaftens. Bielefeld: Kamphausen, S. 136-156.

Ferreira, Y. (2009). FEAT - Fragebogen zur Erhebung von Arbeitszufriedenheitstypen. In: Zeitschrift für Arbeits- und Organisationspsychologie, 53, S. 177-193.

Fleuridas, C. \& Krafcik, D. (2019). Beyond Four Forces: The Evolution of Psychotherapy. SAGE open. Abrufbar auf: https://journals.sagepub.com/doi/pdf/10.1177/215824401882 4492. Abgerufen am: 30.2.2020.

Fueglistaller, U., Fust, A., Müller, C., Müller, S. \& Zwellweger, T. (2019). Entrepreneurship. Modelle - Umsetzung - Perspektiven. Mit Fallbeispielen aus Deutschland, Österreich und der Schweiz (5., überarbeitete Auflage). Springer Gabler: Wiesbaden.

Fürst, M., \& Wieland, J. (2004). WerteManagementSysteme in der Praxis. Erfahrungen und Ausblicke. In: J. Wieland (Hrsg.), Handbuch Werte Management. Erfolgsstrategien einer modernen Corporate Governance. Hamburg: Murmann, S. 595-640.

Gagné, M., Forest, J., Gilbert, M.-H., Aube, C., Morin, E. \& Malorni, A. (2010). The Motivation at Work Scale: Validation Evidence in Two Languages. In: Educational and Psychological Measurement, 70 (4), S. 628-646.

Gillwald, K. (2000). Konzepte sozialer Innovation. WZB Paper: Querschnittsgruppe Arbeit und Ökologie. Berlin: Wissenschaftszentrum Berlin für Sozialforschung. Abrufbar auf: https://bibliothek.wzb.eu/pdf/2000/p00-519.pdf. Abgerufen am 14.4.2020.

Grawe, K. (1998). Psychologische Therapie. Göttingen: Hogrefe.

Grimes, M., Williams, T. \& Zhao, E. (2019). Anchors Aweigh. The Sources, Variety, and Challenges of Mission Drift. In: The Academy of Management Review, 44 (4), S. 819-845.

Harbrecht, A. (2010). Social Entrepreneurship - Gewinn ist Mittel, nicht Zweck. Eine Untersuchung über Entstehung, Erscheinungsweisen und Umsetzung. Karlsruhe: KIT Scientific Publishing.

Hauschildt, J., Salomo, S., Schultz, C. \& Kock, A. (2016). Innovationsmanagement (6., vollständig aktualisierte, überarbeitete Auflage). München: Franz Vahlen.

Heckhausen, H. \& Gollwitzer, P. (1987). Thought contents and cognitive functioning in motivational versus volitional states of mind. Motivation and Emotion, 11 (2), S. 101-120.

Heckhausen, H., Gollwitzer, P. \& Weinert, F. (Hrsg.) (1987). Jenseits des Rubikon. Der Wille in den Humanwissenschaften. Berlin: Springer.

Heckhausen, H. \& Kuhl, J. (1985). From wishes to action. The dead ends and short cuts on the long way to action. In: M. Frese \& L. Sabini (Hrsg.), Goaldirected behavior. Psychological theory and research on action. Hillsdale (USA): Erlbaum, S. 134-159.

Herzberg, F. (1959). The Motivation to Work. New York (USA): Willey.

Hinsz, V. \& Jundt, D. (2005). Exploring individual differences in a goal-setting situation using the motivational trait questionnaire. In: Journal of Applied Social Psychology, 35, S. 551-571.

Hoffmann, P., Scharpe, K. \& Wunsch, M. (2021). 3. Deutscher Social Entrepreneurship Monitor 2020/21. Abrufbar auf: https://www.send-ev.de/wp-content/uploads/2021/03/DSEM2020-21.pdf. Abgerufen am: 25.3.2021.

Hollenbeck, J. \& Brief, A. (1987). The effects of individual differences and goal origin on goal setting and performance. In: Organizational Behavior and Human Decision Processes, 40, S. 392-414. 
Hough, L. \& Furnham, A. (2003). Use of Personality Variables in Work Settings. In: W. Borman, D. Ilgen und R. Klimoski (Hrsg.), Handbook of Psychology (Band 12: Industrial and Organizational Psychology). Hoboken (USA): Wiley, S. 131-170.

Howaldt, J. \& Schwarz, M. (2015). Innovation neu denken - „Soziale Innovation“ als Kern eines neuen Innovationsverständnisses. In: Sozialwissenschaften und Berufspraxis, 38 (2). S. 159-176.

Ickes, W., Snyder, M. \& Garcia, S. (1997). Personality influence on the choice of situations. In: R. Hogan, J. A. Johnson und S. R. Briggs (Hrsg.), Handbook of Personality Psychology. San Diego (USA): Academic Press, S. 165-195.

Jacobsen, L. (2003). Bestimmungsfaktoren für Erfolg im Entrepreneurship - Entwicklung eines umfassenden Modells. Dissertation am Fachbereich Erziehungswissenschaft und Psychologie der Freien Universität Berlin. Abrufbar auf: https://refubium.fu-berlin.de/ bitstream/handle/fub188/13068/00_jacobsen.pdf?sequence=1\&isAllowed=y. Abgerufen am: 4.3.2019.

Jarrodi, H., Byrne, J. \& Bureau, S. (2019). A Political Ideology Lens on Social Entrepreneurship Motivations. In: Entrepreneurship and Regional Development, 31 (7-8), S. 583-604.

Judge, T. \& Ilies, R. (2002). Relationship of personality to performance motivation: A metaanalytic review. In: Journal of Applied Psychology, 87, S. 797-807.

Judge, T., Bono, J., Erez, A. \& Locke, E. (2005). Core self-evaluations and job and life satisfaction: The role of self-concordance and goal attainment. In: Journal of Applied Psychology, 90, S. 267-268.

Jung, C. \& Morner, M. (2016). Das Glasperlenspiel der intrinsischen Motivation - Führungskräfte zwischen gemeinsamen Werten und eigener Verantwortung (Enhancing Intrinsic Motivation Through Shared Values and Self-responsibility). In: Zeitschrift für Wirtschaftsund Unternehmensethik - Journal for Business, Economics \& Ethics, 17 (2), S. 236-258.

Kanfer, R., Chen, G. \& Pritchard, R. (2012). Work motivation. Past, present and future. The organizational frontiers series. New York (USA): Routledge.

Kanter, B. \& Sherman, A. (2016). The Happy, Healthy Nonprofit. Strategies for Impact without Burnout. New Jersey (USA): Wiley.

Katz, R. \& Allen, T. (1982). Investigating the Not Invented Here (Nih) Syndrome. A Look at the Performance, Tenure, and Communication Patterns of $50 \mathrm{R} \&$ D Project Groups. In: R \& D Management, 12 (1), S. 7-19.

Kearney, C., Dunne, P. \& Wales, W. (2020). Entrepreneurial orientation and burnout among healthcare professionals. In: Journal of Health Organization and Management, 34 (1), S. 16-22.

Kelly, G. (1965). Der Motivationsbegriff als irreführendes Konstrukt. In H. Thomae (Hrsg.), Die Motivation des menschlichen Handelns. Köln \& Berlin: Kiepenheuer \& Witsch, S. 498-509.

Kerr, S., Kerr, W. \& Xu, T. (2017). Personality Traits of Entrepreneurs: A Review of Recent Literature. Harvard Business School (Working Paper 18-047), Boston (USA): Selbstverlag.

Kimmitt, J. \& Muñoz, P. (2018). Sensemaking the 'social' in social entrepreneurship. In: International Small Business Journal: Researching Entrepreneurship, 36 (8), S. 859-886. 
Klein, H., Wesson, M., Hollenbeck, J. \& Alge, B. (1999). Goal commitment and the goalsetting process: Conceptual clarification and empirical synthesis. In: Journal of Applied Psychology, 84, S. 885-896.

Kleinbeck, U. \& Schmidt, K.-H. (1996). Die Wirkung von Zielsetzungen auf das Handeln. In: H. Heckhausen und J. Kuhl (Hrsg.), Enzyklopädie der Psychologie, Bd. 4: Motivation, Volition und Handlung. Göttingen: Hogrefe, S. 875-907.

Kleinbeck, U. \& Schmidt, K.-H. (Hrsg.) (2004). Arbeitspsychologie. Enzyklopädie der Psychologie. Bd. D/III/1. Göttingen: Hogrefe, S. 139-177.

Kluger, A. \& DeNisi, A. (1996). The Effects of Feedback Interventions on Performance. A Historical Review, a Meta-Analysis, and a Preliminary Feedback Intervention Theory. In: Psychological Bulletin, 119 (2), S. 254-284.

Komarraju, M. \& Karau, S. (2005). The relationship between the Big Five personality traits and academic motivation. In: Personality and Individual Differences, 39, S. 557-567.

Korber, M. (2015). Innenwelten und Außenwelten von Social Entrepreneurs. Wie Träume unternehmerisch verwirklicht werden können. Wien (Österreich): Facultas.

Krause, F. \& Storch M. (2010). Ressourcen aktivieren mit dem Unbewussten. Manual und ZRM-Bildkartei. Bern (Schweiz): Hans Huber.

Krieger, A. (2017). Skill Variety as a Driver of Entrepreneurship. Dissertation am Fachbereich IV, Wirtschafts- und Sozialwissenschaften, der Universität Trier. Abrufbar auf: https://ubt.opus.hbz-nrw.de/opus45-ubtr/frontdoor/deliver/index/docId/815/file/ Dissertation_Krieger_Final.pdf. Abgerufen am: 4.3.2019.

Kruse, P., Wach, D., Costa, S. \& Moriano J. A. (2018). Values matter, don't they? Combining Theory of Planned Behavior and Personal Values as Predictors of Social Entrepreneurial Intention. In: Journal of Social Entrepreneurship, 10 (1), S. 55-83.

Kruse, P., Wach, D., Wegge, J. (2018). What motivates social entrepreneurs? A meta-analysis on predictors of social entrepreneurial intention. Technische Universität Dresden. Unveröffentlichtes Manuskript, nachgereicht zur gleichnamigen Veranstaltung am 17.09.2018 beim 51. DGPs Kongress an der Goethe-Universität in Frankfurt am Main. Session A6: Unternehmertum, Social Entrepreneurial Intention (SEI). Dozent: Philipp Kruse, Fakultät Psychologie an der Technischen Universität Dresden, Professur für Arbeits- und Organisationspsychologie.

Lambrechts, W., Caniëls, M., Molderez, I., Venn, R. \& Oorbeek, R. (2020). Unraveling the Role of Empathy and Critical Life Events as Triggers for Social Entrepreneurship. Frontiers in Psychology, 11, Aritkel 579500. DOI: https://doi.org/10.3389/fpsyg.2020.579500/ full.

Lapierre, J.-W. (1977). Vivre sans État? Essai sur le pouvoir politique et l'innovation sociale. In: L'Homme, 19 (2), S. 79-81.

Latham, G., Seijts, G. \& Crim, D. (2008). The Effects of Learning Goal Difficulty Level and Cognitive Ability on Performance. In: Canadian Journal of Behavioural Science, 40 (4), S. 220-229.

Lazear, E. (2004). Balanced Skills and Entrepreneurship. In: American Economic Review, 94, S. 208-211.

Lazear, E. (2005). Entrepreneurship. In: Journal of Labor Economics, 23 (4), S. 649-680.

LeDoux, J. (2000). Emotion Circuits in the Brain. In: Annual Review of Neuroscience, 23, $155-184$. 
Lepoutre, J., Justo, R., Terjesen, S. \& Bosma, N. (2013). Designing a global standardized methodology for measuring social entrepreneurship activity: the Global Entrepreneurship Monitor social entrepreneurship study. In: Small Business Economics, 40, S. 693-714.

Leppert, T. (2013). Social Entrepreneurship in Deutschland. Einflussfaktoren auf den Gründungsprozess von Social Entrepreneurs. Hamburg: Kovač.

Leutner, F., Ahmetoglu, G., Akhtar, G. \& Chamorro-Premuszic, T. (2014). The Relationship between the Entrepreneurial Personality and the Big Five Personality Traits. In: Personality and Individual Differences, 63, S. 58-63.

Little, B. (1989). Personal Projects Analysis. Trivial Pursuits, Magnificent Obsessions, and the Search for Coherence. In: D. Buss und N. Cantor (Hrsg.), Personality psychology: Recent trends and emerging directions. New York (USA): Springer, S. 15-31.

Locke, E. (1975). Personnel attitudes and motivation. In: Annual Review of Psychology, 26, S. $457-480$.

Locke, E. \& Latham, G. (1990). A theory of goal setting and task performance. Englewood Cliffs (USA): Prentice Hall.

Locke, E. \& Latham, G. (2002). Building a practically useful theory of goal setting and task motivation: A 35-year odyssey. In: American Psychologist, 57, S. 705-717.

Locke, E. \& Shaw, K. (1984). Atkinson's inverse-U curve and the missing cognitive variables. In: Psychological Reports, 55, S. 403-412.

Locke, E., Shaw, K., Saari, L. \& Latham, G. (1981). Goal setting and task performance: 1969-1980. In: Psychological Bulletin, 90, S. 125-152.

Locke, E., Motowidlo, S. \& Bobko, P. (1986). Using self-efficacy theory to resolve the conflict between goal setting theory and expectancy theory in organizational behavior and industrial organizational psychology. In: Journal of Social \& Clinical Psychology, 4, S. $328-338$.

Lord, W. (2011). Das NEO-Persönlichkeitsinventar in der berufsbezogenen Anwendung, Interpretation und Feedback. Göttingen: Hogrefe.

Luhmann, N. (1984). Soziale Systeme. Grundriß einer allgemeinen Theorie. Frankfurt: Suhrkamp.

Luhmann, N. (2011). Einführung in die Systemtheorie (6. Auflage, Transkription zur gleichnamigen Vorlesung im Wintersemester 1991/92 an der Universität Bielefeld, herausgegeben von Dirk Baecker). Heidelberg: Carl Auer.

Mair, J., \& Martí, I. (2006). Social entrepreneurship research: A source of explanation, prediction, and delight. In: Journal of World Business. 41 (1), S. 36-44.

Majumdar, S. \&. Ganesh, U. (2020). Qualitative Research in Social Entrepreneurship: A Critique. In: S. Majumdar und E. Reji (Hrsg.), Methodological Issues in Social Entrepreneurship Knowledge and Practice. Singapur (Singapur): Springer Nature, S. 15-38.

Marshall, R. (2011). Conceptualizing the International For-Profit Social Entrepreneur. In: Journal of Business Ethics, 98, S. 183-198.

Maslow, A. (1943). A Theory of Human Motivation. Psychological Review, 50 (4), S. 370396. Mit Zugriff auf eine ungekürzte Neuausgabe des Originals von 1999, New Delhi (Indien): General Press, ohne Seitenangabe, mit Absatzangabe.

Maslow, A. (1943a). Self-Actualization and Beyond. Conference on the Training of Counselors of Adults vom 22.-28.5.1943. New England Board of Higher Education \& Center for the Study of Liberal Education for Adults. Chatham \& Brookline (USA): Selbstverlag.

Maslow, A. (1954). Motivation and Personality. New York (USA): Harper \& Row. 
Maslow, A. (1962). Toward a Psychology of Being. New York (USA) \& London (Großbritannien): Van Nostrand. Mit Zugriff auf eine ungekürzte Neuausgabe des Originals von 2014, New York (USA): Start Publishing, ohne Seitenangabe, mit Absatzangabe.

Maslow, A. (1971). The Farther Reaches of Human Nature. New York (USA): Viking.

Maxwell, N., Dunn, A., Rotz, D. \& Shoji, M. (2019). Doing good while doing business: Using financial viability to enhance employability for the disadvantaged. In: Nonprofit Management and Leadership, 29, S. 589-600.

Metzger, G. (2019). Social Entrepreneurs in Deutschland: Raus aus der Nische -154.000 ,junge“ Sozialunternehmer im Jahr 2017. KfW Research, Fokus Volkswirtschaft, Nr. 238, 6. Januar 2019. Abrufbar auf: https://www.kfw.de/PDF/Download-Center/Konzer nthemen/Research/PDF-Dokumente-Fokus-Volkswirtschaft/Fokus-2019/Fokus-Nr.-238Januar-2019-Sozialunternehmer.pdf. Abgerufen am: 5.9.2019.

Meyer, K. (2020). Persönlichkeit, Selbststeuerung und Schlüsselkompetenzen erfolgreicher Unternehmerinnen. Mit erziehungswissenschaftlichen Implikationen. Wiesbaden: Springer Gabler.

Miculaiciuc, A. (2019). Social Entrepreneurship: Evolutions, Characteristics, Values And Motivations. In: Annals of Faculty of Economics, University of Oradea, 1 (1), S. 63-71.

Miller, T., Grimes, M., McMullen, J. \& Vogus, T. (2012). Venturing for others with heart and head: how compassion encourages social entrepreneurship. In: Academy of Management Review, 37 (4), S. 616-640.

Miller, T., Wesley, C. \& Williams, D. (2012). Educating the minds of caring hearts. Comparing the views of practitioners and educators on the importance of social entrepreneurship competencies. In: Academy of Management Learning \& Education, 11 (3), S. 349-370.

Miner, J. (2015). Organizational behavior 1: Essential theories of motivation and leadership. New York (USA): Routledge.

Morris, M., Santos, S. \& Kuratko, D. (2020). The great divides in social entrepreneurship and where they lead us. Small Business Economics. DOI: https://doi.org/10.1007/s11187-02000318-y.

Morrison, E. W. \& Robinson, S. (1997). When employees feel betrayed. A model of how psychological contract violation develops. In: The Academy of Management Review, 22 (1), S. 226-256.

Murray, H. (1938). Explorations in personality. A clinical and experimental study of 50 men of college age. New York (USA): Oxford University.

Neck, H., Brush, C. \& Allen, E. (2009). The Landscape of Social Entrepreneurship. In: Business Horizons, 52 (1), S. 13-19.

Nerdinger, F. (2013). Arbeitsmotivation und Arbeitshandeln. Eine Einführung. Kröning: Asanger.

Nerdinger, F. (2019). Arbeitsmotivation und Arbeitszufriedenheit. In: F. Nerdinger, G. Blickle und N. Schaper (Hrsg.), Arbeits- und Organisationspsychologie (4., vollständig überarbeitete Auflage). Berlin: Springer, S. 463-486.

Neuloh, O. (1977). Zum Bezugsrahmen von sozialer Innovation und sozialem Konflikt. In: O. Neuloh (Hrsg.), Soziale Innovation und sozialer Konflikt. Göttingen: Vandenhoeck \& Ruprecht, S. 9-30.

Neyer, F. \& Asendorpf, J. (2018). Psychologie der Persönlichkeit (6., vollständig überarbeitete Auflage). Berlin: Springer. 
Norman, A. (1993). Informational Society. An Economic Theory of Discovery, Invention and Innovation. Boston (USA): Kluwer.

Norman, A. \& Shimer, D. (1994). Risk, uncertainty, and complexity. In: Journal of Economic Dynamics and Control, 18 (1), S. 231-249.

Ogburn, W. \& Gilfillan, S. (1933). The influence of invention and discovery. In: Research Committee on Social Trends (Hrsg.), Recent Social Trends in the United States. Report of the President's Research Committee on Social Trends, Band 1. New York (USA) \& London (Groß Britannien): McGraw-Hill, S. 122-166. Abrufbar auf: https://archive.org/ details/recentsocialtren01 unitrich/page/162/mode/2up?q=Klux. Abgerufen am: 2.1.2020.

Oldenburg, F. (2011). Wie Social Entrepreneurs wirken - Beobachtungen zum Sozialunternehmertum in Deutschland. In: H. Hackenberg und S. Empter (Hrsg.), Social Entrepreneurship - Social Business. Für die Gesellschaft unternehmen. Wiesbaden: Springer Fachmedien, S. 119-132.

Olenga Tete, P., Wunsch, M. \& Menke, C. (2018). Deutscher Social Entrepreneurship Monitor 2018. Abrufbar auf: https://www.send-ev.de/uploads/dsem-2018_web.pdf. Abgerufen am: 8.3.2019.

Ordóñez, L., Schweitzer, M., Galinsky, A. \& Bazerman, M. (2009). Goals Gone Wild: The Systematic Side Effects of Over-Prescribing Goal Setting. In: Academy of Management Perspectives, 23, S. 6-16.

Organ, D. (1988). Organizational citizenship behavior. The good soldier syndrome. Lexington (USA): Lexington Books.

Osbelt, K. (2019). Social Entrepreneurship - Entstehung und Bedeutung. Abrufbar auf: https://www.send-ev.de/uploads/definition_socialentrepreneurship.pdf. Abgerufen am: 2.1.2020.

Osterloh, M. \& Frey, B. (2000). Motivation, Knowledge Transfer, and Organizational Forms. In: Organization Science, 11 (5), S. 538-550.

Ouchi, W. (1979). A Conceptual Framework for the Design of Organizational Control Mechanisms. In: Management Science, (25) 9, S. 833-848.

Pangriya, R. (2019). Hidden aspects of social entrepreneurs' life: a content analysis. Journal of Global Entrepreneurship Research, 9, Artikel 66. DOI: https://doi.org/10.1186/s40497019-0199-6.

Parker, P. \& Martin, A. (2011). Clergy motivation and occupational well-being: Exploring a quadripolar model and its role in predicting burnout and engagement. In: Journal of Religion and Health, 50, S. 656-674.

Perls, F., Hefferline, R. \& Goodman, P. (1951). Gestalt Therapy. Excitement and Growth in the Human Personality. New York (USA): The Julian Press.

Pestoff, V. \& Hulgård, L. (2016). Participatory Governance in Social Enterprise. In: International Journal of Voluntary and Nonprofit Organizations, 27, S. 1742-1759.

Porter, M. \& Kramer, M. (2011). Creating shared value. In: Harvard Business Review, 89 (1-2), S. 62-77.

Quinn, R. \& Rohrbaugh, J. (1983). A Spatial Model of Effectiveness Criteria: Towards a Competing Values Approach to Organizational Analysis. In: Management Science, 29 (3), S. 363-377.

Rogers, C. (1961). On Becoming a Person. Boston (USA): Houghton Mifflin.

Rogers, E. (1983). Diffusion of Innovation (3. Auflage). New York (USA): The Free Press. 
Roth, G. (2003). Fühlen, Denken, Handeln. Wie das Gehirn unser Verhalten steuert (neue, vollständig überarbeitete Ausgabe). Frankfurt am Main: Suhrkamp.

Roth, G. (2019). Warum es so schwierig ist, sich und andere zu ändern: Persönlichkeit, Entscheidung und Verhalten (13., vollständig überarbeitete Neuauflage). Stuttgart: KlettCotta.

Roth, G. \& Dicke, U. (2005). Funktionelle Neuroanatomie des limbischen Systems. In: H. Förstl, M. Hautzinger und G. Roth (Hrsg.), Neurobiologie psychischer Störungen. Heidelberg: Springer, S. 1-74.

Rummel, M. (2011). Wer sind Social Entrepreneurs in Deutschland? Soziologischer Versuch einer Profilschärfung. Wiesbaden: Springer Fachmedien.

Ruskin, J., Seymour, R. \& Webster, C. (2016). Why Create Value for Others? An Exploration of Social Entrepreneurial Motives. In: Journal of Small Business Management, 54 (4), S. 1015-1037.

Ryan, R. \& Deci, E. (2000). Self-Determination Theory and the Facilitation of Intrinsic Motivation, Social Development, and Well-Being. In: American Psychologist, 55 (1), S. $68-78$.

Sachse, R. (2020). Persönlichkeitsstörungen verstehen. Zum Umgang mit schwierigen Klienten (11. Auflage). Köln: Psychiatrie Verlag.

Scharpe, K. \& Wunsch, M. (2020). Deutscher Social Entrepreneurship Monitor 2019. Abrufbar auf: https://www.send-ev.de/uploads/DSEM2019.pdf. Abgerufen am: 18.2.2020.

Schmidt, K.-H. (2010). Leistungsbeurteilung, Leistungsfeedback und Feedbackwirkungen. In: U. Kleinbeck und K.-H. Schmidt (Hrsg.), Arbeitspsychologie. Enzyklopädie der Psychologie, Bd. D/III/1. Göttingen: Hogrefe, S. 139-177.

Schmidt, K.-H. \& Kleinbeck, U. (1999). Funktionsgrundlagen der Leistungswirkungen von Zielen bei der Arbeit. In: M. Jerusalem und R. Pekrun (Hrsg.), Emotion, Motivation und Leistung. Göttingen: Hogrefe, S. 291-304.

Schneider, K. \& Schmalt, H. (2000). Motivation (3. Auflage). Stuttgart: Kohlhammer.

Schultheiss, O. \& Brunstein, J. (1999). Goal imagery: Bridging the gap between implicit motives and explicit goals. In: Journal of Personality, 67 (1), S. 1-38.

Schumpeter, J. (1947). The Creative Response in Economic History. In: The Journal of Economic History, 7 (2), S. 149-159.

Schwartz, S. (1992). Universals in the Content and Structure of Values. Theory and Empirical Tests in 20 Countries. In: M. Zanna (Hrsg.), Advances in Experimental Social Psychology. New York (USA): Academic Press, S. 1-65.

Schwartz, S. (1994). Are There Universal Aspects in the Structure and Contents of Human Values? In: Journal of Social Issues, (50) 4, S. 19-45.

Schwartz, S. (2003). European Social Survey Core Questionnaire Development. Kap. 7: A Proposal for Measuring Value Orientations across Nations. London (Großbritannien): European Social Survey, City University London.

Schwartz, S. (2011). Studying Values: Personal Adventure, Future Directions. Journal of Cross-Cultural Psychology, 42 (2), S. 307-319.

Schwartz, S. (2012). An Overview of the Schwartz Theory of Basic Values. Online Readings in Psychology and Culture, 2 (1), S. 1-20.

Schweitzer, M., Ordóñez, L. \& Douma, B. (2004). Goal setting as a motivator of unethical behavior. In: Academy of Management Journal, 47, S. 422-432. 
Schwingenstein, M. (2013). Der Sozialunternehmer. Kulturwissenschaftliche Analyse einer Leitfigur postmaterieller Ökonomie. München: Herbert Utz.

Seijts, G. \& Latham, G. (2001). The effect of distal learning, outcome, and proximal goals on a moderately complex task. In: Journal of Organizational Behavior, 22, S. 291-307.

Sheldon, K. \& Elliot, A. (1999). Goal striving, need satisfaction, and longitudinal well-being: The self-concordance model. In: Journal of Personality and Social Psychology, 76, S. 482497.

Storch, M. \& Krause, F. (2014). Selbstmanagement - ressourcenorientiert. Grundlagen und Trainingsmanual für die Arbeit mit dem Zürcher Ressourcen Modell (ZRM®) (5., erweiterte und vollständig überarbeitete Auflage). Bern (Schweiz): Hans Huber.

Storch, M. \& Kuhl, J. (2013). Die Kraft aus dem Selbst (2. Auflage). Bern (Schweiz): Hans Huber.

Ţigu, G., Iorgulescu, M-C., Răvar, A. \& Lile, R. (2015). A Pilot Profile of the Social Entrepreneur in the Constantly Changing Romanian Economy. In: Amfiteatru Economic, 17 (38), S. 25-43.

Tintor, M. (2012). Beschäftigte. Kostenfaktor oder Humankapital? Gesundheitspolitik während betrieblicher Veränderungsprozesse. In: K.-J. Bruder, C. Bialluch, und B. Leuterer (Hrsg.), Macht - Kontrolle - Evidenz. Psychologische Praxis und Theorie in den gesellschaftlichen Veränderungen Gießen: Psychosozial, S. 137-157.

Tuominen-Soini, H. (2012). Student Motivation and Well-Being. Achievement Goal Orientation Profiles, Temporal Stability, and Academic and Socio-Emotional Outcomes. Dissertation am Fachbereich Erziehungswissenschaften an der Universität Helsinki, Institut für Verhaltenswissenschaften. Abrufbar auf: https://www.researchgate.net/ publication/233987557_Student_motivation_and_well-being_Achievement_goal_orie ntation_profiles_temporal_stability_and_academic_and_socio-emotional_outcomes. Abgerufen am: 4.1.2020.

Vancouver, J., Thompson, C., Tischner, E. \& Putka, D. (2002). Two studies examining the negative effect of self-efficacy on performance. Journal of Applied Psychology, 87, S. 506516.

Wagner, J. (1994). Participation effects of performance and satisfaction: A reconsideration of research evidence. In: The Academy of Management Review, 19 (2), S. 312-330.

Winters, D. \& Latham, G. (1996). The effect of learning versus outcome goals on a simple versus a complex task. In: Group and Organization Management, 21, S. 235-250.

Wood, R., Mento, A. \& Locke, E. (1987). Task complexity as a moderator of goal effects. A meta-analysis. In: Journal of Applied Psychology, 72, S. 416-425.

Zabel, H.-U. (2011). Verhaltensmodellierung für Nachhaltigkeit unter besonderer Berücksichtigung empirischer Befunde. In: Zeitschrift für Umweltpolitik und Umweltrecht, 34 (3), S. 339-358.

Zapf, W. (1989). Über soziale Innovationen. In: Soziale Welt, 40 (1-2), S. 170-183.

Zhao, H. \& Seibert, S. (2006). The Big Five personality dimensions and entrepreneurial status. A meta-analytical review. In: Journal of Applied Psychology, 91 (2), S. 259-271.

Zhao, H., Seibert S. \& Lumpkin, G. (2010). The Relationship of Personality to Entrepreneurial Intentions and Performance: A Meta-Analytic Review. In: Journal of Management, 36, S. 381-404. 
Open Access Dieses Kapitel wird unter der Creative Commons Namensnennung 4.0 International Lizenz (http://creativecommons.org/licenses/by/4.0/deed.de) veröffentlicht, welche die Nutzung, Vervielfältigung, Bearbeitung, Verbreitung und Wiedergabe in jeglichem Medium und Format erlaubt, sofern Sie den/die ursprünglichen Autor(en) und die Quelle ordnungsgemäß nennen, einen Link zur Creative Commons Lizenz beifügen und angeben, ob Änderungen vorgenommen wurden.

Die in diesem Kapitel enthaltenen Bilder und sonstiges Drittmaterial unterliegen ebenfalls der genannten Creative Commons Lizenz, sofern sich aus der Abbildungslegende nichts anderes ergibt. Sofern das betreffende Material nicht unter der genannten Creative Commons Lizenz steht und die betreffende Handlung nicht nach gesetzlichen Vorschriften erlaubt ist, ist für die oben aufgeführten Weiterverwendungen des Materials die Einwilligung des jeweiligen Rechteinhabers einzuholen. 